\title{
The Interplay of Progestins, Matrix Metalloproteinases, and the Aging Brain
}

\author{
Keyana Nicole Porter \\ West Virginia University, knporter@mix.wvu.edu
}

Follow this and additional works at: https://researchrepository.wvu.edu/etd

Part of the Cognitive Neuroscience Commons, Molecular and Cellular Neuroscience Commons, Other Neuroscience and Neurobiology Commons, and the Other Pharmacy and Pharmaceutical Sciences Commons

\section{Recommended Citation}

Porter, Keyana Nicole, "The Interplay of Progestins, Matrix Metalloproteinases, and the Aging Brain" (2020). Graduate Theses, Dissertations, and Problem Reports. 7973.

https://researchrepository.wvu.edu/etd/7973

This Dissertation is protected by copyright and/or related rights. It has been brought to you by the The Research Repository @ WVU with permission from the rights-holder(s). You are free to use this Dissertation in any way that is permitted by the copyright and related rights legislation that applies to your use. For other uses you must obtain permission from the rights-holder(s) directly, unless additional rights are indicated by a Creative Commons license in the record and/ or on the work itself. This Dissertation has been accepted for inclusion in WVU Graduate Theses, Dissertations, and Problem Reports collection by an authorized administrator of The Research Repository @ WVU.

For more information, please contact researchrepository@mail.wvu.edu. 


\title{
The Interplay of Progestins, Matrix Metalloproteinases, and the Aging Brain \\ Keyana N. Porter
}

\begin{abstract}
Dissertation submitted to the School of Pharmacy at West Virginia University

in partial fulfillment of the requirements for the degree of
\end{abstract}

Doctor of Philosophy

in

Pharmaceutical and Pharmacological Sciences

\author{
James W. Simpkins, Ph.D., Advisor \\ Marina Galvez-Peralta, Pharm. D., Ph.D. \\ Ann F. Hubbs, Ph.D. \\ Candice M. Brown, Ph.D. \\ Werner J. Geldenhuys, Ph.D. \\ Department of Pharmaceutical Sciences \\ Morgantown, West Virginia \\ 2020
}

Keywords: matrix metalloproteinases, medroxyprogesterone acetate, amyloid beta $(A \beta)$, amyloid beta degradation, Alzheimer's disease, hormone therapy, glucocorticoid receptor 


\section{Abstract \\ The Interplay of Progestins, Matrix Metalloproteinases, and the Aging Brain}

Keyana N. Porter

Progestins are synthetic hormones that are designed to mimic the biological actions of progesterone. They, however, possess other pharmacological actions and properties, in addition to their progestational activities. Medroxyprogesterone Acetate (MPA) is a progestin used globally in the hormonal contraceptive, Depo Provera ${ }^{\circledR}$, by women in their reproductive prime and is a major compound found in hormone therapy (HT) formulations used by menopausal women. MPA is used by approximately 1 in 5 adolescents and adult women in the United States who are sexually active. Globally, nearly 48 million women utilize injectable contraceptives to prevent pregnancy, with most users utilizing MPA as their hormone of choice. Despite the extensive use of hormonal methods as either contraception or menopausal $\mathrm{HT}$, there is very little known about the potential effects of these compounds on the cellular processes of the brain. Additionally, MPA promotes changes in the circulating levels of matrix metalloproteinases (MMPs), such as MMP-9, in the endometrium, yet limited literature studying the effects of MPA on neurons and astroglia cells has been conducted. Furthermore, the dysregulation of MMPs has been implicated in the pathology of Alzheimer's disease (AD), where inhibiting the secretion of MMP-9 from astroglia reduces the proteolytic degradation of amyloid beta.

The key objective for my dissertation work was to investigate the effects of acute and chronic administration of MPA on MMP-9 production and secretion, as well as AD-related pathology, by utilizing hormonal modulation of $A \beta$-degrading enzymes. Two sets of studies were designed to begin addressing some of the knowledge gaps associated with MPA and its potential effects on the brain. The hypothesis of these studies was that MPA alters the levels of MMP-9 secretion and enzymatic activity, in turn, negatively impacting the degradation of $A \beta$ and cognitive function.

The first set of studies examined the outcomes of MPA on MMP-9 secretory, proteolytic, and $A \beta$-degrading activities. We found that MPA treatment inhibited transcription of MMP-9, which resulted in a subsequent decrease in the production and secretion of MMP-9 protein, in part through the glucocorticoid receptor. Additionally, we investigated the consequences on amyloid beta-degrading activity and found that MPA treatment decreased proteolytic degradation of amyloid beta. Our results suggest MPA suppresses amyloid beta degradation in an MMP-9dependent manner, in vitro, and potentially compromises the clearance of amyloid beta in vivo.

The second set of studies was an in vivo assessment, evaluating the effects of chronic administration of exogenous progestins on cognitive function and MMP-9 expression. Our findings suggest that long-term, subcutaneous administration of MPA negatively impacts cognitive function, specifically memory consolidation, in wild-type (WT) mice, and enhances cognitive function, in a triple transgenic mouse model of AD (3xTg-AD), but causes a net increase MMP-9 expression in both mouse models.

Collectively, these two studies demonstrated that MPA's actions on the brain need to be further investigated and more inclusive of non-menopausal and AD models. MPA elicits a differential effect in WT and AD animals, however, increases cortical MMP-9 expression in both phenotypes. Overall, these findings suggest that MPA has the potential to elicit differential effects on women in their reproductive prime and women with predispositions for AD and should encourage more elaborate investigations of its effects. 


\section{Acknowledgments}

I would like to thank everyone that l've been blessed and privileged to have as a member of my support system. Without you all, the work presented in this dissertation would not have been possible.

First and foremost, my family deserves endless thanks for enduring the challenges of the past several years alongside me. My graduate studies revealed to me how extraordinary my upbringing has been. I would like to thank my parents and grandparents for their unconditional selflessness and countless sacrifices, which have opened many doors for me. Much of my success has been a direct result of the work ethic and value system that you all instilled in me. I want to give a special acknowledgement to my biological father, Harvey Porter III, whom is no longer with us. As his only child, his legacy lives on through me and my determination to uphold his legacy is what makes me the intensely motivated woman I am today. To the rest of my family, thank you for your unwavering support and tolerating my absence at many holiday dinners and family functions. Keith, thank you for teaching me patience and unwavering faith that God is always working for me, even when I fall short of seeing it for myself.

Next, I must thank my advisor and mentor, Dr. James W. Simpkins. You have always supported my creativity and imagination by allowing me to conceive and develop my own studies, while also empowering me to overcome the many experimental setbacks I've encountered during my matriculation. You encourage me to nourish my interests outside of the lab, in turn, increasing the professional opportunities I've been afforded. Your mentorship fosters intrinsic motivation and a perfect level of autonomy, which has ultimately transformed me into a goal-driven, productive professional and researcher.

I am so grateful for the current and former members of the Simpkins lab, including Dominic Quintana, Ashley Russell, Deborah Corbin, Stephanie Rellick, Elizabeth EnglerChiurazzi, Sophie Ren, John Cavendish, Sara Lewis, Sujung Jun, and Heng Hu. I want to give a special thanks to Dr. Saumyendra Sarkar who was a close mentor and scientific advisor, and whose wisdom and insight was indispensable to my research training. Thank you for pushing me to become more of a critical thinker and challenging my scientific curiosity. Collectively, each one of you helped to increase my sense of belonging at WVU and helped to make this journey bearable.

Several faculty members and staff at WVU have also greatly impacted my scientific journey. I would like to thank Dr. Candice Brown, Dr. Marina Galvez-Peralta, Dr. Ann Hubbs, and Dr. Werner Geldenhuys, my dissertation committee members, for their guidance, expertise, and engagement. Thank you to Dr. Lisa Salati for being invested in my success and continuously challenging me to fulfill my greatest potential. I would like to thank Duaa Dakhlallah for providing extensive guidance and strengthening the impact of my data. 
Additionally, thank you Cassandra George and Joseph Andria for your administrative support throughout my matriculation. Your abilities to troubleshoot and resolve any research-related issues I encountered outside of the laboratory not only made life less hectic, but also made me feel supported and genuinely care for, which undoubtedly contributed to my success in the lab.

Lastly, I owe many thanks to my friends that have supported me throughout my scientific career. Thank you to Skye Smith, Lindsey Bishop, Ashley Lindo, Ahnieyah Porter, Emily Sechrest, Kristina Whately, Divine Nwafor, Allison Brichacek, Tanya Dilan, Rachel Hostetler, Catherine Smith, Ray Anderson, Deidre O'Dell, Dylan Boehm, and finally, my sorority sisters of the Alpha Gamma Chapter, Zeta Epsilon Chapter, and the Omicron Upsilon Chapter of Delta Sigma Theta, Sorority, Inc.

If anyone was left out of these acknowledgements, please know that I am grateful for your help!

Black lives matter. 


\section{Table of Contents}

CHAPTER 1: INTRODUCTION \& LITERATURE REVIEW

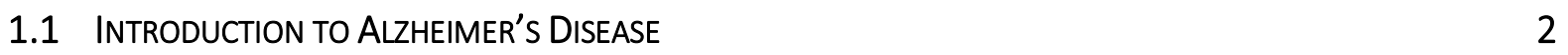

1.1.1 EPIDEMIOLOGY AND IMPACT 2

1.1.2 PROGRESSION OFAD 2

1.2 RISK FACTORS FOR AD 4

1.2.1 GENETIC AND ENVIRONMENTAL RISK FACTORS 4

1.2.2 GENDER-AND SEX-SPECIFIC RISK FACTORS 8

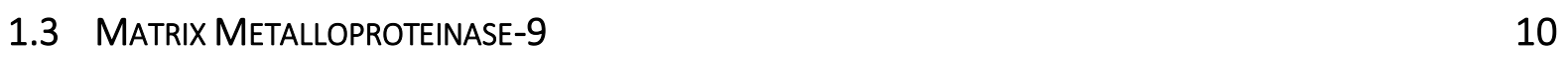

1.3.1 INTRODUCTION TO MATRIX METALLOPROTEINASES (MMPS) 10

$\begin{array}{ll}\text { 1.3.2 Structure, EXPRESSION, AND ACtiVAtION } & 11\end{array}$

1.3.3 REgULATION 12

1.4 Matrix Metalloproteinase-9 (MMP-9) AND Brain Function 13

1.4.1 MMP-9 AND INFLAMMATION 13

$\begin{array}{lll}\text { 1.4.2 MMP-9 AND STROKE } & 14\end{array}$

1.4.3 MMP-9 AND SYNAPTIC PLASTICITY 15

$\begin{array}{ll}\text { 1.4.4 MMP-9 AND AD } & 15\end{array}$

$\begin{array}{lll}1.5 & \text { Female Reproductive AgING } & 17\end{array}$

1.5.1 INTRODUCTION TO THE FEMALE REPRODUCTIVE SYSTEM 17

$\begin{array}{ll}1.6 & \text { ADOLESCENCE \& ADULTHOOD } \\ 1.6 & 18\end{array}$

$\begin{array}{ll}\text { 1.6.1 SenESCENCE } & 19\end{array}$

$\begin{array}{lll}1.7 & \text { Progestogens } & 20\end{array}$

1.7.1 Progesterone 20

$\begin{array}{ll}\text { 1.7.2 SYNTHETIC PROgestins } & 22\end{array}$

1.8 HORMONES AND COGNITIVE DECLINE

1.8.1 ESTROGENS 23

$\begin{array}{ll}\text { 1.8.2 ANDROGENS } & 25\end{array}$

1.9 Knowledge Gap: Progestogens and Cognitive Function 26

2 CHAPTER 2: MEDROXYPROGESTERONE ACETATE IMPAIRS AMYLOID BETA DEGRADATION IN A MATRIX METALLOPROTEINASE-9 DEPENDENT MANNER 30

$\begin{array}{lll}2.1 & \text { ABSTRACT } & 31\end{array}$

2.2 INTRODUCTION 32

2.3 Materials AND MEthods $\quad 35$

$\begin{array}{lll}2.4 & \text { RESULTS } & 43\end{array}$

$\begin{array}{lll}2.5 & \text { DISCUSSION } & 48\end{array}$

$\begin{array}{lll}2.6 & \text { FIGURES } & 56\end{array}$ 
3 CHAPTER 3: THE IN VIVO EFFECTS OF LONG-TERM ADMINISTRATION OF MEDROXYPROGESTERONE ACETATE ON MATRIX METALLOPROTEINASES IN COGNITIVE FUNCTION

3.1 ABSTRACT

3.2 INTRODUCTION

3.3 MATERIALS AND METHOdS

74

3.4 RESULTS

3.5 DISCUSSION

80

3.6 FIGURES

87

4 CHAPTER 4: GENERAL DISCUSSION

95

5 REFERENCES 


\section{List of symbols, abbreviations, and nomenclature}

17ß-estradiol (E2)

activator protein 1 (AP1)

Alpha-secretase ( $\alpha$-secretase)

Alzheimer's Disease (AD)

Amyloid beta $(A \beta)$

Amyloid beta 1-40 $\left(A \beta_{1-40}\right)$

Amyloid beta 1-42 (A $\left.\beta_{1-42}\right)$

Amyloid precursor protein (APP)

Amyotrophic lateral sclerosis (ALS)

androgen receptor (AR)

angiotensin-converting enzyme (ACE)

apolipoprotein (APOE4)

Beta-secretase ( $\beta$-secretase)

beta-site APP Cleaving Enzyme 1 (BACE1)

Blood-brain barrier (BBB)

brain-derived neutrophic factor (BDNF)

Central nervous system (CNS)

cerebrospinal fluid (CSF)

conditioned media (CM)

conjugated equine estrones (CEE)

dimethylsulfoxide (DMSO)

Early onset Alzheimer's (EOAD)

endothelin-converting enzyme (ECE)

Enzyme-linked immunosorbent assay (ELISA)

estrogen receptor (ER)

extracellular matrices (ECM)

Familial Alzheimer's disease (FAD)

follicle-stimulating hormone (FSH)

Frontotemporal Lobar Degeneration (FTLD)

Gamma-secretase ( $\gamma$-secretase)

glucocorticoid receptor (GR)

glucocorticoid response element (GRE)

gonadotropin releasing hormone $(\mathrm{GnRH})$

Hidden platform (HP)

Hormone therapy (HT)

human T-lymphotropic virus type 1 (HTLV-1)

insulin-degrading enzyme (IDE)

interleukin $1 \alpha(\mathrm{IL}-1 \alpha)$

lactate dehydrogenase (LDH)

Late onset AD (LOAD)

Lewy Body dementia (LBD) 
long term potentiation (LTP)

luteinizing hormone (LH)

Matrix Metalloproteinase-2 (MMP-2)

Matrix Metalloproteinase-3 (MMP-3)

Matrix Metalloproteinase-9 (MMP-9)

Matrix Metalloproteinases (MMPs)

medroxyprogesterone acetate (MPA)

membrane progesterone receptors (mPR)

messenger RNA (mRNA)

microRNA (miR)

mineralocorticoid receptor (MR)

multiple sclerosis (MS)

neprilysin (NEP)

Neurofibrillary tangles (NFT)

nuclear factor kappa B (NFkB)

nuclear factor kappa B (NF-kB)

ovariectomized (OVX)

perineuronal net (PNN)

peripheral nervous system (PNS)

prefrontal cortex (PFC)

Presenilin 2 (PSEN2)

Presenilin1 (PSEN1)

Progesterone (P4)

progesterone receptors (PR)

progesterone response element (PRE)

Reversal (Rev)

serum-free media (SFM)

soluble APP $\alpha$ (SAPP $\alpha)$

Sporadic Alzheimer's disease (SAD)

tissue inhibitors of MMPs (TIMP)

tissue plasminogen activator (tPA)

Traumatic brain injury (TBI)

triple transgenic mouse model of Alzheimer's disease (3xTg-AD)

tumor necrosis factor $\alpha$ (TNF- $\alpha)$

Vascular Dementia (VaD)

Visible platform (VP)

Wild-type (WT)

Women's Health Initiative Memory Study (WHIMS) 


\section{Chapter 1}

Introduction \& Literature Review 


\subsection{Introduction to Alzheimer's Disease}

\subsubsection{Epidemiology and Impact}

Dementia is an "umbrella" term used to describe a range of symptoms associated with cognitive impairment. The four most common types of dementia are Alzheimer's disease (AD), Vascular Dementia (VaD), Frontotemporal Lobar Degeneration (FTLD), and Lewy Body dementia (LBD). Within these four types of dementia, AD is the most prevalent. $A D$ is an age-related, progressive dementia and the sixth leading cause of death in the United States (U.S.). AD is also the only disease in the top 10 causes of death in the U.S. that currently has no way of being prevented, cured, or delayed. It is also the only disease in the U.S. top 10 causes of death whose mortality rate consistently and substantially increases, showing a $145 \%$ increase since the year 2000 . There are 5.8 million Americans that are estimated to be currently living with $A D$, with 5.6 million over the age of 65 . With current estimates, the number of people, age 65 and older with, AD may triple to nearly 14 million by 2050 , which emphasizes the need for developments and advances towards slowing or curing this disease. Overall, $A D$ is progressive in nature, responsible for increased deaths, and contributes to poor health outcomes and disabilities in the U.S., which consequently is a huge burden on patients, caregivers, the healthcare system, and the economy.

\subsubsection{Progression of $A D$}

The progression of AD can be divided into three stages: mild, moderate, and severe (Burns and Iliffe, 2009). A patient can experience their first symptoms of mild memory loss before the age of 65 (early onset AD, EOAD) and over the age of 65 (late onset AD, LOAD). Morphologically, the cerebral cortex and subcortical regions are atrophied in AD brains. The most important neuropathological phenomena relevant to $A D$ is the progressive 
accumulation of extracellular deposits of amyloid beta (amyloid plaques; $A \beta$ ); aggregates of intracellular tau protein (neurofibrillary tangles); and loss of neurons and synapses (reviewed by Haass et al., 2007). These changes are also accompanied by glial activation, neurite dystrophy, and neuronal death (Jović et al., 2019).

Extracellular amyloid plaques are mostly composed of $A \beta$ peptide, which is generated by proteolytic cleavage of amyloid precursor protein (APP) (De Strooper, 2010). APP is recognized by alpha-secretase ( $\alpha$-secretase), which cleaves the precursor protein, promoting the non-amyloidogenic cleavage of APP, which has been shown to promote neuroprotection and memory enhancement (Ghiso and Frangione, 2002). However, as reviewed by Chen et al., (2015), with aging, $\alpha$-secretase becomes progressively inefficient. This causes APP to be truncated by non-specific proteases such as beta- and gammasecretase ( $\beta$-secretase and $\psi$-secretase), promoting the amyloidogenic cleavage of APP (Holsinger et al., 2002; Yang et al., 2003; Zhang et al., 2014; Carroll and Li, 2016). Cleavage of APP at the $\beta$ - and $\psi$-secretase sites produces the 40 or 42 amino acid fragments of A $\beta$ ( $A \beta_{1-40}$ and $A \beta_{1-42}$ ) and subsequently, $A \beta$ peptide is released into the extracellular space (Chen, 2015; Xu et al., 2016). Extracellular $A \beta$ assumes several conformational states ranging from monomers to soluble oligomers and fibrils. These polymers of $A \beta$ quickly aggregate and form the amyloid plaques which are characteristic of the disease (Pryor et al., 2012). Aggregates of $A \beta$, colocalized with activated microglia, is additionally observed early in the development of AD symptoms (Meda et al., 1995; Cagnin et al., 2001). The leading hypothesis regarding the causation of AD-related neurodegeneration attributes abnormal $A \beta$ accumulation as a key initiating event (Hardy and Selkoe et al., 2002; Hardy et al., 2009). 
Tau is a microtubule-associated protein encoded by the MAPT gene. Mutations in MAPT have been reported to impact the age of onset of AD. Stabilization of cytoskeletal microtubules of axons occurs through the interaction of tubulin and tau protein. Under pathological conditions, tau is abnormally phosphorylated at multiple residues on the protein, destabilizes cytoskeletal microtubules, and in turn, weakens the cytoskeleton to a degree that increases neuronal vulnerability. Hyperphosphorylation of tau eventually aggregates and forms pathological intracellular deposits (neurofibrillary tangles, NFT). The severity of clinical symptoms has been shown to be highly correlated with the progressive accumulation of NFTs, and there is no correlation between clinical symptoms and deposition of $A \beta$ (Kauwe et al., 2008). However, aberrant tau phosphorylation is reported to be stimulated by $A \beta^{\prime}$ s modulation of protein kinases and phosphatases that regulate the phosphorylation of tau (Binder et al., 1985; Grundke-lqbal et al., 1986; Arriagada et al., 1992; Götz et al., 2001; Bloom et al., 2014; Wilkaniec et al., 2016). Alternatively, Leroy et al. (2012) demonstrated that the absence of tau decreased amyloid plaque burden by nearly $50 \%$. Collectively, the literature suggests there is a strong relationship between the amyloidogenic processing of APP and tau protein.

\subsection{Risk Factors for AD}

\subsubsection{Genetic and environmental risk factors}

Multifactorial in nature, $A D$ is caused by heredity and environmental factors. The rate of progression of $A D$ is increased by the existence of $A D$ in a family's history (familial $A D, F A D)$, promoted by mutations in the amyloid precursor protein (APP) and presenilin 1 (PSEN1) and 2 (PSEN2) genes. Mutations in these genes severely impacts the metabolism of 
$A \beta$ (production, aggregation, and clearance) (Murakami et al., 2003; Tsubuki et al., 2003). Notably, the majority of these mutations are linked to PSEN1/2 mutations (Sherrington et al., 1993). Of the mutations in the APP gene, the majority were identified near the binding sites of the secretases responsible for cleaving APP and generating A $\beta$, thus affecting APP processing and leading to increased $A \beta$ deposition. Familial $A D$ is dominantly inherited, with clinical symptoms surfacing by the age of 45 (Scheltens et al., 2016).

The majority of $A D$ cases are sporadic (sporadic $A D, S A D$ ) in nature. Although the primary causes of SAD are not extensively known, there are several social and environmental factors that have been identified to interact with genetic risk factors, which influence the onset and progression of the disease. Aging is the highest risk factor for AD, but lifestyle differences such as smoking, diet, and physical exercise, and engagement in cognitive activities, influenced by education and occupation, also contribute to the risk for AD (Mielke et al. 2014). Level of education, for example, has been shown to promote cognitive reserve, or cognitive resilience, which is thought to serve as a protective mechanism against cognitive decline and influence susceptibility to AD-related pathology (Stern et al., 2009).

Stroke has also been shown to be a major risk factor for dementias, such as $A D$ and Vascular dementia (VaD). Post-mortem brain analyses of $A D$ brains have revealed a strong correlation between a history of stroke and more severe dementia (Snowdon et al., 1997). Furthermore, a history of stroke has also been shown to be strongly associated with an earlier onset of dementia (Honig et al., 2003). It is currently proposed that stroke worsens AD-related symptoms, but also, AD increases the risk for stroke. The inflammatory cascade 
initiated by stroke-related insults more than likely results in brain damage that potentiates, and maybe even exacerbates, the progression of AD (Honig et al., 2003). Alternatively, AD potentially increases the risk for stroke, by predisposing the brain to pathological and molecular changes, that substantiate the risk for stroke. In fact, stroke, $\mathrm{VaD}$, and $A D$ share common risk factors including aging, hypertension, insulin resistance, diabetes, obesity, and hyperlipidemia (Craft et al., 2009). Thus, stroke and dementia possess an additive or synergistic relationship.

Traumatic brain injury (TBI) is also closely related to neurodegeneration and dementia, both pathologically and metabolically. Cerebrovascular injury has been shown to induce $A \beta$ and tau deposition, ultimately causing cognitive impairments and AD-related pathology (Iadecola, 2013; Pluta et al., 2013; De Silva and Faraci, 2016; Wolters et al., 2017; Tarasoff-Conway et al., 2015). Herein, TBI promotes further damage to endothelial cells, alterations in the levels of tight junction proteins and MMP, and a disruption in blood-brain barrier (BBB) permeability (Wen et al., 2014; Salehi et al., 2017; Abdul-Muneer et al., 2013). Mitochondrial dysfunction is a consequence of TBI and has also been suggested to be a contributing and potentiating factor in AD (Silva et al., 2013; Fossati et al., 2016; Swerdlow et al., 2018; Szarka et al., 2018). However, the link between mitochondrial dysfunction and TBI have not been extensively investigated.

In addition to metabolic risk factors for $S A D$, there are genetic risk factors that have been identified to be highly associated with SAD. For example, beta-site APP Cleaving Enzyme 1 (BACE1), a unit component of $\beta$-secretase is also positively associated with the risk for SAD. Genomic studies have revealed additional molecular factors that contribute to 
increased risk for SAD through their regulatory functions of immunological responses to neuroinflammation (TREM2, CLU, CR1, ABCA7, CD33, EPHA1, and the MS4A family), synaptic function (PICALM, BIN1, CD33, CD2AP, and EPHA1), and transport and metabolism of cholesterol (APOE, SORL1, CLU and ABCA7) (Seshadri et al., 2010; Hollingworth et al., 2011; Jiang et al., 2013; Totso and Reitz, 2013; Griciuc et al., 2013; Hickman et al., 2014; Rosenthal et al., 2015; Ising et al., 2015).

There is an increased risk and accelerated onset of $A D$, with the presence of the $\varepsilon 4$ allele of the apolipoprotein (APOE4) gene, which is the greatest known genetic risk factor for SAD (Corder et al., 1993; Saunders et al., 1993). Outside of cholesterol transport, APOE plays an important role in neuroplasticity and APP processing. It binds $A \beta$, negatively impacting clearance mechanisms and thus, promotes aggregation. Genes involved in the regulation of inflammatory responses and cholesterol metabolism were highly correlated with clinical and neuropathological symptoms of AD (Koldamova et al., 2005; Kim et al., 2009; Castellano et al., 2011; Goedeke et al., 2014). Carriers of APOE4 have also been shown to develop AD at an earlier age, and simply carrying a single allele of this gene has been found to nearly triple the risk of AD (Corder et al., 1993; Saunders et al., 1993; Strittmatter et al.,1993).

In general, age-related changes in gene expression are due to epigenetic regulation. This includes DNA methylation, histone modifications, and changes in the expression of microRNA (miR). miR are small, non-coding single-stranded RNA molecules that regulate the expression of genes that are in involved in AD. Several miR have been shown to be heavily involved in direct regulation of APP expression in FAD (Delay et al., 2001; Patel et al., 2008; 
Hébert et al., 2009; Vilardo et al., 2010; Long et al., 2011; Long et al., 2012; Schonrock et al., 2012). Similarly, genes attributed to SAD have been shown to be regulated by miR. The gene which encodes the active unit of $\alpha$-secretase (ADAM10), for example, is regulated by miR that are altered in peripheral blood of AD patients (Postina et al., 2008; Augustin et al., 2012; Leidinger et al., 2013). In theory, utilizing miRNA as biomarkers can aid in the early diagnosis and therapeutic approaches for both FAD and SAD (Kiko et al., 2014). Further understanding of these molecular factors at the RNA and protein levels will ultimately lead to an improved understanding in the interactions of these genes and their close association to the symptoms in $A D$.

\subsubsection{Gender- and sex-specific risk factors}

The Institute of Medicine (2001) defines sex as the biological and physiological differences between men and women, attributed to the sex chromosomes ( $\mathrm{X}$ and $\mathrm{Y}$ ). Gender-specific differences refer to environmental, social, and cultural influences men and women, adding more of an experiential effect to the biological effects. There is a clear gender disparity that exists in the incidence of $A D$. Collectively, the risk for $A D$ is influenced by factors with the same frequency for both men and women, but a stronger effect in one particular sex, factors that have similar effects in men and women, but different frequencies, and factors that are different both in strength and frequency, Women are disproportionately affected with AD, when compared to men. Of the 5.6 million people age 65 and older who are impacted by $A D, 3.5$ million are women and 2.1 million are men (Herbert et al. 2013). AD also progresses faster in woman than men (Tschanz et al. 2011), and women experience significantly more severe pathology than men (Ruitenberg et al., 
2001; Corder et al., 2004; Barnes et al., 2005; Schmidt et al., 2008; Sinforiani et al., 2010;

Chapman et al., 2011; Hall et al., 2012; Irvine et al., 2012). Several explanations have been proposed to explain the sex difference in AD. Women are twice as likely to develop depression and live more sedentary lifestyles, which are known risk factors for AD (Kessler et al., 1993; Dotson et al., 2010; Blondell et al., 2014; Livingston et al., 2017; Singh-Manoux et al., 2017; Horder et al., 2018). Additionally, APOE4, the strongest risk factor for AD, impacts women and men differently, as women with APOE4, have been found to be more likely to develop dementia than men with APOE4 (Farrer et al., 1997; Fleisher et al., 2008; Altmann et al., 2014; Sampedro et al., 2015). However, with age being a major risk factor for $A D$, the most simplistic theory is that women live longer than men, making it appear there are more women living with AD than there are men. This concept of "survival bias" was shown in the Framingham Heart Study, which reported an increased mortality rate in men due to poor cardiovascular health and decreased risk for dementia in men who reached the age of 65 and older, when compared to women (Petrea et al., 2009). Epidemiologists considered the data was impacted by the fact that men with positive cardiovascular health generally living healthier lives, lowering their overall risk factors for dementia.

Sex differences are also observed in several transgenic mouse models of $A D$, where female AD animals have been reported to experience an earlier on-set of neuropathology, as well as more severe AD-related pathology. Accumulation of $A \beta$, for example, is higher in

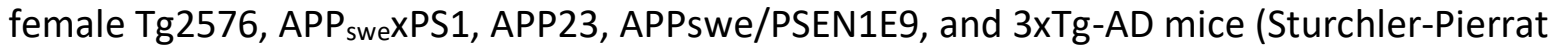
and Staufenbiel, 2000; Callahan et al., 2001; Lee et al., 2002; Anderson et al., 2003; Hirata- 
Fukae et al., 2008; Halford and Russell, 2009; Carroll et al., 2010). Additionally, performances in behavior assays of cognitive function suggest female transgenic AD mice are more cognitively impaired when compared to male mice. The data from these studies suggest there are sex-specific risk factors that exist.

\subsection{Matrix Metalloproteinase-9}

\subsubsection{Introduction to Matrix Metalloproteinases (MMPs)}

The integrity of the central nervous system (CNS) is maintained by the extracellular matrix, which consists of structural and signaling molecules that are essential for cellular differentiation and proliferation. Homeostatic mechanisms that regulate cell migration, extension of neurites, and synaptic plasticity are heavily dependent on proteins secreted into the extracellular matrix, and to maintain homeostasis, these proteins are degraded by proteolytic enzymes. Just as extracellular matrix proteins are regulated, these proteolytic enzymes are also regulated at various levels. Matrix metalloproteinases (MMPs) are zincdependent enzymes that have many extracellular substrates. MMPs are expressed in many cell types, including endothelial cells, muscle cells, and, in the central nervous system (CNS), neurons and glia (Genersch et al., 2000; Roomi et al., 2009). To date, there are over 20 MMPs that have been identified (Ethell and Ethell, 2007), with a few residing in the CNS, in

normal and pathological conditions (MMP-1, MMP-2, MMP-3, MMP-7, MMP-9, MT1-MMP, MT5-MMP, and their inhibitors, TIMP-1,-2,-3, and -4) (Tanney et al., 1998; Vaillant et al., 1999; Oh et al., 1999; Llano et al., 1999; Sekine-Aizawa et al., 2001; Agapova et al., 2001; Rosenberg et al., 2001; Schutz et al., 2002; Costa et al., 2002). 


\subsubsection{Structure, Expression, and Activation}

The majority of MMPs share similar structural domains, which includes an Nterminal signal peptide, a propeptide region, a catalytic domain containing a $\mathrm{Zn}^{2+}$ binding site, a short hinge region, fibronectin repeats and C-terminal hemopexin-like repeats (Hayashita-Kinoh et al., 2001; Sekine-Aizawa et al., 2001). MMPs are differentiated by the components within their hemopexin-like and fibronectin repeats. Uniquely, MMP-9 and MMP-2 are closely related but distinguished from other MMPs by their fibronectin type II inserts, in their catalytic domains. The fibronectin type II inserts allow for MMP-9 and MMP2 to bind elastin, laminin, and collagen, as well as cleave their substrates (Van den Steen et al., 2002). The $\mathrm{N}$-terminal signal peptide targets the enzyme for secretion and promotes the cleavage of the MMPs, for activation. The propeptide region contains a "cysteine switch" sequence, and a disruption between the cysteine and $\mathrm{Zn}^{2+}$ interaction initiates activation of the MMP (Vallee and Auld, 1990). MMPs are regulated by the propeptide region, which maintains the enzymes in an inactive state by binding tissue inhibitors of MMPs (TIMP) (Cao et al., 1998; Pavlaki et al., 2002).

Intracellularly, MMP-9 is distributed along microtubules and microfilaments, via vesicular transport. Secretion of MMP-9 occurs via the ER-Golgi secretory pathway, in $200-$ $1000 \mathrm{~nm}$ vesicles. Initially, MMP-9 is secreted extracellularly as a catalytically inactive proenzyme (proMMP-9). The pro-form of MMP-9 is incapable of cleaving its substrates until the proteolysis, or protein unfolding, of the active site occurs. ProMMP-9 is bound to TIMP1 at the C-terminal domain of MMP-9. Once the "cysteine switch" occurs, the proMMP9•TIMP complex dissociates with the assistance of other MMPs, tissue plasminogen 
activator (tPA)-plasmin system, and/or serine proteases, allowing proMMP-9 to become fully activated (MMP-9) (Stawarski et al., 2014). Some MMPs require assistance from membrane type MMPs (MT-MMPs). MMP-2, for example, has a secretory mechanism which involves a tertiary complex with TIMP-2 and MT1-MMP.

\subsubsection{Regulation}

The expression, secretion, and proteolytic activities of MMPs are tightly regulated, during early development and adulthood, at three levels: transcription, activation, and inhibition by physiological inhibitors. There are also post-translational modifications, compartmentalization, and storage mechanisms that additionally regulate MMP activity. MMP-9 mRNA, protein, and enzymatic levels have been detected in several regions of the CNS, such as the hippocampus, brainstem, cortex, and cerebellum (Oliveira-Silva et al., 2007; Gawlak et al., 2009; Aujla and Huntley, 2014; Bednarek et al., 2014). MMP-9 is mainly expressed in glia and along oligodendrocyte processes (Dzwonek et al., 2004; Gawlak et al., 2009). The expression of MMPs also appears to be function-specific more than cell-specific, for example, protein expression and enzymatic activity of MMP-9 appears localized to cell bodies, dendrites, and excitatory synapses that are found on dendritic spines.

In the adult brain, MMP-9 levels are ubiquitously low, and altered in response to various stimuli, including growth factors, cytokines, metal ions, oncogenes, and hormones (reviewed by Van den Steen et al., 2002). The signaling pathways shown to be involved in the activation of MMPs are ERK-MAP kinase, phosphoinositide 3 (PI-3) kinase, protein kinase C (PKC), and p38 pathways (White et al., 2000; Wang et al., 2002; Hussain et al., 
2002). There are also binding sites for activator protein 1 (AP1), nuclear factor kappa B

(NFKB), Smad, and Ets in the promoter sequence of most MMPs.

Importantly, TIMP expression is critical for preventing excessive MMP proteolytic

activity. TIMPs appear to be regulated in a similar manner, through the binding of the same nuclear factors that bind MMPs. Due to similar release kinetics, TIMP expression is also inversely related to the expression of MMPs (Sato et al., 2002; Zechel et al., 2002). The literature suggests MMP-9 is specifically inhibited by TIMP-1, via direct binding of TIMP-1 to the catalytic site of MMP-9 or direct binding of MMP-9's hemopexin domain to the Cterminus of TIMP-1. Ultimately, the tight regulation of MMP-9's proteolytic activity is essential to its regulatory functions in the CNS.

\subsection{Matrix Metalloproteinase-9 (MMP-9) and Brain Function}

MMPs possess both beneficial and detrimental impacts on brain development, the adult brain, and the spinal cord. Collectively, MMPs have been implicated in BBB dysfunction, demyelination, neuroinflammation, neurotoxicity, and CNS diseases such as multiple sclerosis (MS) and stroke. The literature shows MMP-9 holds a unique and essential role in the physiology and pathology of many brain functions and dysfunctions.

\subsubsection{MMP-9 and Inflammation}

Neuroinflammation typically induces immune response mediators to be released from microglia, the resident macrophages in the brain, as well as neurons and astrocytes. A dysfunctional BBB promotes increased leukocyte infiltration, as well as increased MMP-9 production. Conversely, MMP-9, has been shown to induce proinflammatory cytokines (Candelario-Jalil et al., 2009), which also influences BBB permeability, through the cleavage 
of the vascular basal lamina and/or tight junctions between cells belonging to the neurovascular unit (Reijerkerk et al., 2006; Candelario-Jalil et al., 2009; Barkus et al., 2010; Verslegers et al., 2013). This MMP-9-induced BBB disruption serves as the foundation for the pathogenesis of MS, where increased MMP-9 expression and activity have been consistently detected in the serum, cerebrospinal fluid (CSF), and demyelinating lesions of

multiple sclerosis (MS) patients (Yong et al., 2007). The literature also suggests MMP-9 plays a role in bacterial and viral infections. During human T-lymphotropic virus type 1 (HTLV-1) infection in glial cells, for example, tumor necrosis factor $\alpha$ (TNF- $\alpha$ ) and interleukin $1 \alpha$ (IL$1 \alpha$ ) yielded increased MMP-9 expression and secretion in glial cells and in CSF (Giraudon et al., 1996).

\subsubsection{MMP-9 and Stroke}

There is also an abundance of literature, demonstrating MMP-9's role in stroke (Chaturvedi and Kaczmarek, 2014; Yang and Rosenberg, 2015). Within the multiple roles MMP-9 plays in stroke, it mainly influences infarct volume and post-stroke recovery. Genetic ablation of MMP-9 reportedly protects against ischemic stroke (Jin et al., 2010; Rivera et al., 2010). Currently, tPA is the only FDA-approved treatment for ischemic stroke. The limitation of this treatment is its success depends on a highly specific 4.5-hour time window. If administered more than 4.5 hours post-onset of the stroke, MMP-9 is activated, which, in turn, causes further disruption of the BBB (Murata et al., 2008). This ultimately worsens post-stroke outcomes, highlighting the important role of MMP-9 in stroke outcome. 


\subsubsection{MMP-9 and Synaptic Plasticity}

MMP-9's beneficial role in synaptic plasticity is well documented. Dendritic spine morphology is highly dependent on MMP-9 expression and activity (Bilousova et al., 2009; Benson and Huntley, 2012), where overexpression of MMP-9 yields thinner dendritic spines (Michaluk et al., 2011). Additionally, there are several ex vivo and in vivo studies that show MMP-9 maintains long term potentiation (LTP), and inhibition of MMP-9 leads to LTP impairment, within multiple pathways (Nagy et al., 2006; Meighan et al., 2006; Bozdagi et al., 2007; Okulski et al., 2007; Conant et al., 2010; Wojtowiscz and Mozrymas, 2010; Dziembowska et al., 2012; Wiera et al., 2012; Gorkiewicz et al., 2015). MMP-9 has also been shown to be important, during postnatal periods, for the development of sensory circuits and the regulation of synaptogenesis and myelination (Oh et al., 1999; Michaluk et al., 2009; Peixoto et al., 2011). Pharmacological inhibition or genetic deletion of MMP-9 also negatively impacts learning and memory. Localization of MMP-9 expression and activity has been shown to be behavior task-dependent. For example, expression and activity were found to be increased in the hippocampus, prefrontal cortex, piriform cortex, and cerebellum, following the performance of the head-shake response task (Wright et al., 2006), while increased MMP-9 expression was reported in the hippocampus, frontal cortex, and amygdala after the completion of contextual fear conditioning and passive avoidance (Nagy et al., 2007; Ganguly et al., 2013). Collectively, MMP-9 expression is crucial for healthy synaptic function and learning and memory.

\subsubsection{MMP-9 and $A D$}

MMP-9 is also heavily implicated in neurogenerative disease, including Parkinson's disease, Huntington's disease, Down syndrome, Amyotrophic lateral sclerosis (ALS), and AD 
(Yong, 2005). Regarding AD, there is a clear link between MMP-9 and AD pathogenesis.

MMP-9 expression is stimulated by the presence of $A \beta$ peptide (Deb and Gottschall et al., 1996), and may serve to maintain a homeostatic balance between the production and clearance of $A \beta$. There are several zinc-metalloproteinases that mediate the clearance of $A \beta$, including endothelin-converting enzyme (ECE), angiotensin-converting enzyme (ACE), insulin-degrading enzyme (IDE), neprilysin (NEP) and matrix metalloproteinases (MMPs) (Saido and Leissring et al., 2012). Among these A $\beta$-degrading enzymes, MMP-9 is the most distinguished, as it has been shown to be capable of degrading a range of $A \beta$ species. Furthermore, deposition of $A \beta$ has been shown to be inversely associated with MMP activity (Lim et al., 1997; Ridnour et al., 2012). Not only has a genetic association been shown (Helbecque et al., 2003), but postmortem analyses of AD brain tissues show increased MMP-9 levels in neurons, neurofibrillary tangles, and senile plaques (Backstrom et al., 1996; Asahina et al., 2001; Helbecque et al., 2003; Lorenzl et al., 2003). Additionally, MMP-9 levels in the CSF of AD patients were shown to be lower than levels in control patients (Mroczko et al., 2014). TIMP-1 has also been shown to be increased in the CSF of AD patients, most likely due to increased MMP-3 levels in the CSF and plasma (Reitz, et al., 2010; Horstmann et al., 2010). An imbalance between MMP-9 and its regulatory enzyme, TIMP-1, is also implicated in AD. AD patients tend to have high MMP-9/TIMP-1 ratios, along with high levels of tau in their CSF (Talamagas et al., 2007).

Along with $A \beta$, tau is also a substrate for MMP-9, as well as MMP-3, which has been shown to be the activator of proMMP-9 (Hernández-Guillamon et al., 2009). Ironically, while MMP-3 has been shown to degrade tau in regions that facilitate fibril formation, MMP-9 has 
been shown to promote aggregation via its tau cleavage sites (Nübling et al., 2012). This is attributed to the differences in their affinities for cleaving the protein. However, as previously mentioned, MMP-3 is a known activator of proMMP-9, which would suggest an increase in brain MMP-3 yields an indirect proaggregatory effect on tau via increased MMP9 activity (Nübling et al., 2012). Moreover, APOE4, a major risk factor for AD, significantly reduces $A \beta$-induced MMP-9 levels. Thus, reduction of astrocytic MMP-9 would most likely impact clearance of $A \beta$ and encourage deposition in AD (Guo et al., 2006; Bell et al., 2012).

\subsection{Female Reproductive Aging}

\subsubsection{Introduction to the female reproductive system}

The female reproductive system is controlled by communication between the hypothalamus, anterior pituitary gland, and ovaries, forming the hypothalamic-pituitaryadrenal (HPA) axis (reviewed by Hiller-Sturmhöfel and Bartke, 1998; reviewed by Del Rio et al., 2018). From the hypothalamus, a small peptide, gonadotropin releasing hormone $(\mathrm{GnRH})$, is secreted and travels to the anterior pituitary gland. Secretion of this peptide regulates the release of the gonadotropins, luteinizing hormone $(\mathrm{LH})$ and follicle-stimulating hormone (FSH), from cells in the anterior pituitary gland, to the ovaries. FSH stimulates the development of ovarian follicles in the ovaries and stimulates the secretion of estrogen by follicle cells, of the ovaries. LH stimulates ovulation and the production of progesterone by the corpus luteum of the ovaries. Once LH reaches a peak/surges, there is a rapid decline in estrogen levels which increases cholesterol receptor levels in the ovaries, more specifically in theca and granulosa cells. This free cholesterol is then converted to progesterone and progesterone exits the cells, binds albumin and corticosteroid-binding albumin and enters 
into circulation. To regulate the release of gonadotropins, there is a negative feedback loop exerted on the hypothalamus established, where high levels of circulating estrogen suppresses further release of FSH and high levels of circulating progesterone suppresses further release of $\mathrm{LH}$.

\subsection{Adolescence \& Adulthood}

Once a woman reaches the menarche, or first menses, gonadotropin levels undergo cyclic changes (reviewed by Boswell, 2014; reviewed by Galvez-Peralta, 2015). The menstrual cycle can be divided into two phases. The first 14 days is known as the follicular phase and the last 14 days is known as the luteal phase. In the follicular phase, there is pulsatile release of $\mathrm{GnRH}$ from the hypothalamus, which stimulates the synthesis and release of FSH and LH from the pituitary gland. FSH will then stimulate the maturation of ovarian follicles, as well as stimulate inhibin production and the conversion of androgens to estrogens (E2) by granulosa cells. Negative feedback on the pituitary and hypothalamus is exerted by E2 and inhibin, which is only expressed when circulating estrogen levels are low. After about 10 days, once high estrogen levels are achieved, there is positive feedback exerted on the pituitary gland, stimulating the release of $\mathrm{LH}$ and triggering ovulation. After ovulation, $\mathrm{LH}, \mathrm{FSH}$, and GnRH levels decrease, and the follicle turns into a corpus luteum. The corpus luteum begins to secrete inhibin and progesterone. Inhibin has a negative feedback and inhibits the secretion of FSH, while progesterone has a negative feedback on the hypothalamus, inhibiting the secretion of $\mathrm{GnRH}$, affecting the release of FSH and $\mathrm{LH}$. Progesterone also stimulates the growth of the endometrial lining. Under the assumption there is no fertilization, the corpus luteum degrades to allow new follicles to undergo 
maturation and progesterone is no longer inhibiting $\mathrm{GnRH}$ release. This causes the endometrial lining to shed (menstruation) and allows for the initiation of the next cycle. The combination of estrogen and progesterone suppresses gonadotropin synthesis and release through their effects on the HPA axis. Estrogen inhibits the release of FSH from the pituitary, in turn inhibiting follicular development. Without the development of ovarian follicles, there is no LH and thus, ovulation will not occur. Progestins in the combination formulation and even in the progestin-only formulations prevent ovulation by inhibiting $\mathrm{LH}$ release. This will prevent the LH surge and subsequently prevent ovulation. Additionally, progestins causes thickening of cervical mucus and endometrial atrophy. The thickening of the cervical mucus prevents sperm penetration and endometrial atrophy interferes with implantation, helping to prevent pregnancy.

\subsubsection{Senescence}

Perimenopause is often referred to as "Menopausal Transition". Ovarian follicles produce estrogen and inhibin, in response to $\mathrm{FSH}$, allowing inhibin to prevent further synthesis and secretion of FSH. The inevitable decrease of ovarian follicles causes a gradual decline in circulating estrogen levels and inhibin production. Decreased inhibin production leads to subsequent loss of the negative feedback loop to the hypothalamus, increasing $\mathrm{GnRH}$ secretion. Women begin to experience irregular menstruation at this stage and these changes in hormonal regulation ultimately leads to a woman's last menstrual period. At the menopausal stage, the ovaries become depleted of ovarian follicles, massively impacting estrogen and inhibin levels to the point a woman no longer ovulates or menstruates. Postmenopause is characterized by the loss of ovarian sensitivity to $\mathrm{FSH}$ and $\mathrm{LH}$, as well as the 
lack of the negative feedback that is typically exerted on the HPA axis, by E2 and inhibin. These changes lead to increased production and release of $\mathrm{GnRH}, \mathrm{FSH}$, and $\mathrm{LH}$, as well as extremely low levels of estrogen. The combination of progesterone and estrogen is used to "normalize" the menstrual cycle, which during the menopausal periods of a woman's life is irregular. Therefore, treatment with the combination of progesterone and estrogen will help with peri-menopausal symptoms. Furthermore, the cyclic use of progesterone, two weeks on, two weeks off, will often keep the normal menstrual cycle going. Daily therapy with $0.625 \mathrm{mg}$ of conjugated equine estrones (CEE) + 2.5-5mg of medroxyprogesterone acetate (MPA) will eliminate cyclic bleeding, control vasomotor symptoms, prevent genital atrophy, maintain bone density, and promote a favorable lipid profile. Progesterone's effects on the pituitary apparently contribute to its protective effect against osteoporosis, hypertension, hirsutism, etc. Thus, the complementation of progestin treatment is used to treat estrogen excess or deficiency to help alleviate vasomotor symptoms.

\subsection{Progestogens}

The term "progestogen" is used to describe both natural progesterone and synthetic derivations of progesterone, or progestins (Stanczyk et al., 2003). MPA is an example of a progestin, found in both birth control formulations and hormone therapies (HT).

\subsubsection{Progesterone}

Progesterone is a well-characterized female reproductive hormone that plays a major role in ovarian functions, such as implantation and menstruation. It is synthesized in the ovary (corpus luteum and granulosa cells), the testis, and the adrenal cortex from circulating cholesterol, as well as the placenta during pregnancy (Hu et al., 2010). The 
reproductive synthesis of progesterone is controlled by communication between the hypothalamus, anterior pituitary gland, and ovaries, forming the hypothalamic-pituitaryovarian axis. A surge in luteinizing hormone (LH) increases free cholesterol, which is ultimately converted to progesterone (Kuo et al., 2012). Once synthesized, progesterone targets the female genital tract, the pituitary gland, mammary glands, osteoblasts, and the CNS (Taraborrelli, 2015). There is overwhelming evidence that the brain synthesizes steroids, like progesterone, de novo from cholesterol by neural cells of both the CNS and peripheral nervous system (PNS) (Reddy, 2010). Almost all brain cholesterol is found in oligodendrocytes and plasma membranes. Thus, progesterone synthesis occurs in oligodendrocytes, astrocytes, glial cells, Purkinje neurons, and in the peripheral nervous system, progesterone synthesis occurs in Schwann cells (Zwain and Yen, 1999). After the initial findings by Zwain and Yen et al., (1999) demonstrating neural cells have the capacity to synthesize steroids, several lines of strong evidence have followed, in both animal and human studies, reporting progesterone is synthesized in these neural cells.

Synthesis of progesterone in the brain modulates intracellular signaling pathways, channels, and transcription, in turn impacting neuronal activity. In neural cells, progesterone binds to intracellular progesterone receptors. These progesterone receptors are either in the nucleus where they regulate the transcription of progesterone-sensitive genes, or they directly interact with membrane-associated kinases (reviewed by Jacobsen and Horwitz, 2013). Progesterone enters the cell and binds to progesterone receptors (PR) that are distributed between the nucleus and the cytoplasm. To activate gene transcription, the ligand-receptor complex binds to a progesterone response element (PRE) in target 
promoters. This leads to an up-regulation or down-regulation of gene transcription and subsequently leads to tissue responses. Progesterone may also act via non-genomic signaling, in which it activates membrane progesterone receptors (mPR), which are usually directly linked to intracellular signal transduction or second messenger systems, mediating rapid tissue responses.

\subsubsection{Synthetic Progestins}

Synthetic progestins are commonly used in place of natural progesterone due to their ability to inhibit ovulation and their greater bioavailability, half-life, and activity postadministration (Pasqualini et al., 1996; Sitruk-Ware et al., 2000). Since 1938, the year the very first progestin was synthesized, many generations of progestins have been synthesized, differing in their chemical structures and biological effects (Inhoffen and Hohlweg, 1938). Structurally, synthetic progestins either relate to progesterone or testosterone and these structural differences influence their relative binding affinity (RBA) to sex steroid receptors, which in turn impacts their transcriptional activity. In addition to the PR, progestins interact with androgen receptor (AR), estrogen receptor (ER), glucocorticoid receptor (GR), and mineralocorticoid receptor (MR). Like progesterone, progestins enter the cell and bind their cognate receptors that are distributed between the nucleus and the cytoplasm. To activate gene transcription, the ligand-receptor complex binds to a hormone response element (PRE) in target promoters, or bind receptors in the cytoplasm, yielding an up-regulation or down-regulation of gene transcription. Furthermore, although natural progesterone holds a strong RBA for mPRs, it is currently inconclusive whether progestins mediate their actions via mPRs. Within a list of 30 steroidal compounds, the RBAs for mPRs failed to exceed $50 \%$ 
(Thomas et al., 2007). The non-genomic membrane bound signaling mechanisms of synthetic progestins remain to be elucidated.

\subsection{Hormones and Cognitive Decline}

Age-related hormonal changes have been found to negatively impact cognitive function and impact risk for AD. There is an abundance of literature which focuses on the effects of estrogen-related compounds, androgens, and progesterone on the brain.

\subsubsection{Estrogens}

The neuroprotective effects of estrogen-related compounds are well-documented in the literature. Estrogens have been reported to protect against neuronal death (Simpkins et al., 2009, 2010, 2012; Suzuki et al., 2009), antagonize AD-related pathology (Pike et al., 2009), and regulate spine density, LTP, and neurotransmitters, in turn, protecting cognitive function (Cooke and Woolley, 2005). Estrogens were initially linked to $A \beta$ once it was reported that E2 modifies APP processing, by either increasing enzymes that reduce levels of $A \beta$ or decreasing enzymes that increase levels of $A \beta$, ultimately promoting the nonamyloidogenic pathway (Desdouits-Magnen et al., 1998; Jaffe et al., 1994; Greenfield et al., 2002; Gandy et al., 2003; Thakur and Mani et al., 2005; Bernstein et al., 2009; Amtul et al., 2010; Nord et al., 2010). Estrogen-related compounds have also been shown to promote A $\beta$ clearance by inducing NEP (Liang et al., 2010; Grimm et al., 2013), IDE (Amtul et al., 2010; Zhao et al., 2011), and transthyretin expression, which sequesters $A \beta$ to prevent aggregation (Schwarzmann et al., 1994; Quintela et al., 2009; Amtul et al., 2010; Oliveira et al., 2011). 
Clinically, there are lower circulating levels of E2 in the blood and the brain, in women with AD, when compared to age-matched controls (Yue et al., 2005; Rosario et al., 2011). Surgical removal of the ovaries from wild-type (WT) female animals, which significantly reduces the levels of circulating E2 and progesterone, has been shown to yield increased levels of $A \beta$ (Petanceska et al., 2000). Many studies have further demonstrated how E2 can prevent and rescue pathology in AD mouse models (Levin-Allerhand et al., 2002; Xu et al., 2002; Carroll et al., 2007; Carroll and Pike et al., 2008;). Hogervorst et al., (2002) sought out to investigate the effect of ET (estrogens alone) versus HT (CEE+ progestogen) on cognitive function in postmenopausal women. From this double-blinded randomized controlled trial, they reported modest positive cognitive outcomes from HT. The following year, the Women's Health Initiative Memory Study (WHIMS) evaluated the cognitive outcomes of post-menopausal HT consisting of CEE with or without MPA. The findings from this large-scale clinical trial suggests HT fails to delay the progression of cognitive decline in women, and revealed HT increases the risk for dementia (Rapp et al., 2003; Shumaker et al., 2003). These findings have encouraged further investigation and discussion of why the discrepancy between basic research and clinical research data exists (Gleason et al., 2015; Henderson et al., 2016). The age in which HT is initiated, the hormonal components within the formulation, and the treatment regimen are suggested to be the most important influential factors on cognitive outcomes (Carroll et al., 2010; Craig and Murphy, 2010; Whitmer et al., 2011). 


\subsubsection{Androgens}

Androgenic compounds have also been shown to hold neuroprotective roles by promoting the growth of neurons, regeneration of axons, and function of synapses (MacLusky et al., 2004; Huppenbauer et al., 2005; Marron et al., 2005; Brannvall et al., 2005; Hajszan et al., 2008; Hatanaka et al., 2009). Additionally, androgens protect against age-related insults and AD-related pathology, such as $A \beta$ toxicity and production, by targeting APP processing and metabolizing enzymes (Gouras et al., 2000; Pike et al., 2001; Nguyen et al., 2005; Park et al., 2007; Zhang et al., 2014; McAllister et al., 2010). Clearance mechanisms of $A \beta$ are also affected by androgenic compounds, where androgens have been shown to increase the expression of NEP, which holds an androgen response element in the promoter region of the NEP gene (Shen et al., 2000; Yao et al., 2008; McAllister et al., 2010). The literature also indicates testosterone regulates circulating $A \beta$ in the CSF and plasma of castrated male animals (Wahjoepramono et al., 2008) and anti-androgen therapy causes an accumulation of circulating $A \beta$ (Almeida et al., 2004). Moreover, age-related depletion of hormones, in the brain, leads to increased levels of $A \beta$, in rat brains (Rosario et al., 2009). In a comparative study, where the effects of various sex steroid hormones on $A \beta$ levels were assessed, testosterone was shown to reduced levels of $A \beta$, to a higher degree, when compared to E2, in castrated 3xTg-AD mice (Rosario et al., 2010). Overall, low levels of testosterone have been linked to increased levels of $A \beta$, worsened cognitive outcomes, impaired brain metabolism, and increased risk of AD (Moffat et al., 2004; Verdile et al., 2012; Tan et al., 2013; Butchart et al., 2013). 


\subsection{Knowledge Gap: Progestogens and Cognitive Function}

As previously mentioned, the term "progestogen" is used to describe both natural progesterone and synthetic derivations of progesterone, or progestins (Stanczyk et al., 2003). Progesterone has been shown to be neuroprotective in stroke, TBI, and neurodegenerative diseases such as AD (Goodman et al., 1996; Jiang et al., 1996; Roof et al., 1996; Chen et al., 1999; Roof et al., 2000; Kumon et al., 2000; Nilsen et al., 2002a,b; Nilsen et al., 2003; Morali et al., 2005; Pettus et al., 2005; Kaur et al., 2007; Singh et al., 2013). Synthetic progestins are utilized by healthy women to control ovarian function earlier in life, and to limit menopausal symptoms later in life. The brain is a major target for synthetic progestins. Due to their lipophilicity, they have been shown to easily crosses the bloodbrain-barrier and rapidly diffuses throughout the CNS, into brain regions such as the hypothalamus, hippocampus, amygdala, and cortex, as well as the spinal cord (Sohrabji, 2007; Wilson et al., 2008; Banks et al., 2012; Diotel et al., 2018). Despite the multiple generations of progestins that have been synthesized, there needs to be further investigation of their effects on brain functions. Notably, the cognitive effects of progestogens, when administered alone, is currently understudied, where majority of investigations study the effects of progestogens when administered with an estrogen.

MPA is an example of a progestin, found in both birth control formulations and HT. MPA, which is used to reduce the risk of cancers resulting from unopposed estrogen therapy, is the most commonly used progestogen in HT regimens (Gambrell et al., 1986; Hirvonen et al., 1996). Despite the fact MPA structurally resembles progesterone more than any other progestin, there are vast differences in their pharmacological and 
pharmacokinetic profiles (Hackenberg et al., 1990; Schindler et al., 2003; Winneker et al., 2003; Koubovec et al., 2005; Jodhka et al., 2009). It has also become increasingly clear that although progesterone is considered neuroprotective, MPA is not. MPA has been shown not only to inhibit brain-derived neutrophic factor (BDNF), which negatively impacts neurogenesis, but also fails to alter glutamate-induced intracellular $\mathrm{Ca}^{2+}$ influx, while progesterone's neuroprotective effects have been shown to be dependent on both neutrophin signaling and attenuation of glutamate-induced increases in $\mathrm{Ca}^{2+}$ (Kaur et al., 2007; Jodhka et al., 2009). The neuroprotective effects of E2 are also antagonized by MPA but substantiated by progesterone (Miyagawa et al., 1997; Rosano et al., 2000; Nilsen et al., 2002b; Littleton-Kearney et al., 2005).

Allopregnanolone, a metabolite of progesterone, is a neuroactive steroid. Dysregulation of allopregnanolone has been associated with the pathophysiology of neurodegenerative disorders, including Alzheimer's disease. Allopregnanolone levels are significantly decreased, both in women and in rats, after long-term administration of hormonal contraceptives, with levels being reduced by almost $80 \%$ (Follesa et al., 2002; Rapkin et al., 2006; Porcu et al. 2012; Santoru et al., 2014). When administered allopregnanolone, 3xTg-AD mice were shown to have improved performances on cognitive tasks through the regeneration of hippocampal nerve cells (Wang et al., 2010). In vitro, allopregnanolone was found to increase the number of neural progenitor cells from the hippocampus of rats and human cerebral cortex (Wang et al., 2005). Additionally, there are several studies which demonstrate restoration of allopregnanolone levels is essential for a therapeutic benefit in AD. These findings suggest that the reduction of allopregnanolone 
may play a significant role in the pathogenesis of AD. It is currently unclear if MPA is a substrate for the progesterone metabolizing enzymes, 5-alpha-reductase and 3-alphahydroxysteroid dehydrogenase. In lieu of MPA's inability to be converted to neuroactive steroid metabolites, coupled with its reduced potential to convert brain progesterone/neuroprogesterone to allopregnanolone, it is evident MPA has limited neuroprotective efficacy.

Clinically relevant exogenous hormones are globally used, but their long-term effects aren't well understood. While limited research with MPA suggests a negative impact on the brain, these studies need to be extended to model systems relevant to women in their reproductive prime, as majority of efforts have been in menopausal animal models and menopausal women (Shumaker et al., 2003, 2004; Braden et al., 2010, 2011, 2017; Lowry et al., 2010; Olanrewaju et al., 2013). Thus, there is an unmet need for studies focusing on the effects of chronically using MPA, earlier in life, and before menopause. Furthermore, as previously stated, estrogens can modulate the levels of $A \beta$-degrading enzymes, such as NEP and IDE. Merlo and Sortino (2012) utilized estrogen to induce the A $\beta$-degrading activity of MMP-9, establishing MMP-2 and MMP-9's contribution to the neuroprotective effect of estrogen, in vitro, and demonstrating estrogen can also modulate the levels of additional $A \beta$-degrading enzymes. There is accumulating evidence, in systems other than the CNS, that MPA can modulate the levels of MMP-9 (Di Nezza et al., 2003; Hwang-Levine et al., 2011; Allen et al., 2019). Thus, investigation of the effects on MPA on MMP-9 secreted from glia remains to be pursued. 
The population of AD patients is progressively increasing, while simultaneously advancing the burden on caregivers, healthcare providers, and the healthcare system. Due to the multifactorial and heterogenous nature of $A D$, the identification of risk factors for $A D$ is essential for understanding how to potentially prevent and delay the progression of this disease. A major issue in the field of $A D$ is nearly two-thirds of $A D$ patients are female. To attribute the gender disparity in AD to survival bias would be logical, however AD-related changes in the brain are initiated nearly 20 years before an official diagnosis, thus the survival bias cannot fully explain the sex- and gender-specific differences (Hof et al., 1996; Perl et al., 2010; Bateman et al., 2012). Collectively, the precise explanation as to why women currently bear the greatest AD burden remains yet to be clearly determined.

The overarching goal of this dissertation work was to characterize the effects of exogenous progestin treatment on AD-related pathology, utilizing hormonal modulation of A $\beta$-degrading enzymes. The first strategy was an in vitro evaluation of the effects of the commonly used progestin, MPA, on MMP-9. Chapter 2 examined the outcomes of MPA on MMP-9 secretory, proteolytic, and A $\beta$-degrading activities. The second strategy was an in vivo assessment, evaluating the effects of chronic administration of exogenous progestins on AD-related cognitive function. Chapter 3 further evaluated the in vivo effects of longterm, subcutaneous administration of MPA on cognitive functions in WT and 3xTg-AD mice. Chapter 4 summarized the findings from the studies evaluating the acute and chronic effects of MPA on AD-related pathology, in vitro and in vivo, and outlines the limitations, important takeaways, and future directions of these studies. 


\section{Chapter 2}

\section{Medroxyprogesterone Acetate Impairs Amyloid Beta Degradation in a Matrix Metalloproteinase-9 Dependent Manner}

Keyana N. Porter, Saumyendra N. Sarkar, Duaa A. Dakhlallah, Mya E. Vannoy, Dominic D. Quintana, and James W. Simpkins

Porter, KN. Sarkar, SN. Dakhlallah, DA. Vannoy, ME. Quintana, DD. Simpkins, JW. 2020. Medroxyprogesterone Acetate Impairs Amyloid Beta Degradation in a Matrix Metalloproteinase-9 Dependent Manner. Front. Aging Neurosci. 12:92. 


\subsection{Abstract}

Despite the extensive use of hormonal methods as either contraception or menopausal hormone therapy $(\mathrm{HT})$, there is very little known about the potential effects of these compounds on the cellular processes of the brain. Medroxyprogesterone Acetate (MPA) is a progestogen used globally in the hormonal contraceptive, Depo Provera, by women in their reproductive prime and is a major compound found in HT formulations used by menopausal women. MPA promotes changes in the circulating levels of matrix metalloproteinases (MMPs), such as MMP-9, in the endometrium, yet limited literature studying the effects of MPA on neurons and astroglia cells has been conducted. Additionally, the dysregulation of MMPs has been implicated in the pathology of Alzheimer's disease $(A D)$, where inhibiting the secretion of MMP-9 from astroglia reduces the proteolytic degradation of amyloid beta. Thus, our hypothesis is that exposure to MPA disrupts proteolytic degradation of amyloid beta through the downregulation of MMP-9 expression and subsequent secretion. To assess the effect of progestins on MMP-9 and amyloid beta, in vitro, C6 rat glial cells were exposed to MPA for 48 hours and then the enzymatic, secretory, and amyloid beta degrading capacity of MMP-9 was assessed from the conditioned culture medium. We found that MPA treatment inhibited transcription of MMP-9, which resulted in a subsequent decrease in the production and secretion of MMP-9 protein, in part through the glucocorticoid receptor. Additionally, we investigated the consequences on amyloid beta-degrading activity and found that MPA treatment decreased proteolytic degradation of amyloid beta. Our results suggest MPA suppresses amyloid beta degradation in an MMP-9-dependent manner, in vitro, and potentially compromises the clearance of amyloid beta in vivo.

Keywords: matrix metalloproteinases, medroxyprogesterone acetate, amyloid beta $(A \beta)$, amyloid beta $(A B)$ degradation, Alzheimer's disease, hormone therapy, glucocorticoid receptor 


\subsection{Introduction}

Alzheimer's disease (AD) is a neurodegenerative disease with three key pathological hallmarks: the progressive accumulation of extracellular deposits (amyloid plaques); aggregates of intracellular protein (neurofibrillary tangles); and loss of neurons and synapses (reviewed by Haas et al., 2007). Extracellular amyloid plaques are mostly composed of amyloid beta peptide (AB), which is generated by proteolytic cleavage of amyloid precursor protein (APP) (De Strooper, 2010). APP is recognized by alpha-secretase ( $\alpha$-secretase), which cleaves the precursor protein, promoting the non-amyloidogenic cleavage of APP, which has been shown to promote neuroprotection and memory enhancement (Ghiso and Frangione, 2002). However, as reviewed by Chen et al., (2015), with aging, $\alpha$-secretase becomes progressively inefficient. This causes APP to be truncated by non-specific proteases such as $\beta$-secretase and $\gamma$-secretase, promoting the amyloidogenic cleavage of APP (Holsinger et al., 2002; Yang et al., 2003; Zhang et al., 2014; Carroll and Li, 2016). Cleavage of APP at the $\beta$ - and $\psi$-secretase sites produces the 40 or 42 amino acid fragments of $A \beta\left(A \beta_{1-40}\right.$ and $\left.A \beta_{1-42}\right)$ and subsequently, $A \beta$ peptide is released into the extracellular space (Chen, 2015; Xu et al., 2016). Extracellular A $\beta$ assumes several conformational states ranging from monomers to soluble oligomers and fibrils. These polymers of $A \beta$ quickly aggregate and form the amyloid plaques which are characteristic of the disease (Pryor et al., 2012).

The clearance of $A \beta$ is mediated by several $A \beta$-degrading zinc-metalloproteinases, including endothelin-converting enzyme (ECE), angiotensin-converting enzyme (ACE), insulindegrading enzyme (IDE), neprilysin (NEP) and matrix metalloproteinases (MMPs) (Saido and 
Leissring, 2012). MMPs are a family of highly regulated, zinc-dependent enzymes that are produced by neurons and astroglia (Dzwonek et al., 2004). These enzymes are initially secreted as catalytically inactive pro-enzymes (proMMP-9), which are bound to endogenous inhibitors, named tissue inhibitors of metalloproteinases (TIMPs), through the C-terminal domain of each molecule. A disturbance of this complex (proMMP-9•TIMP), by a proMMP activator (MMP-3), causes the pro-enzyme (proMMP-9) to dissociate, which becomes fully activated, and proceeds with the degradation of its substrates (Ogata et al., 1995;

Rosenblum et al., 2007). Functionally, MMPs are extremely diverse, with regulatory roles in many important cellular processes including synaptic plasticity and cognition, neuroinflammation, blood-brain barrier (BBB) integrity, cell migration, survival and apoptosis, as reviewed by Vafadari et al., (2016). MMPs have been found to be overexpressed during various pathological conditions such as stroke, epilepsy, schizophrenia, and neurodegeneration (Vafadari et al., 2016). Their expression can be induced by a large variety of factors, including cytokines, growth factors, metal ions, antibiotics, and hormones (Van Den Steen et al., 2002; Vandooren et al., 2017). In the context of $A D, A \beta$ exposure induces the expression and secretion of MMPs from cultured neurons, reactive astroglia, and neuroblastoma cells (Deb and Gottschall, 1996; Deb et al., 2003; Talamagas et al., 2007; Mizoguchi et al., 2009). When secreted from astroglia, proMMP-9 is immediately cleaved into an active form (MMP-9) in the extracellular compartment, thus contributing to the maintenance of the balance between $A \beta$ production and clearance (Ogata et al., 1995; Akiyama et al., 2000; Wegiel et al., 2000). In contrast to other $A \beta$-degrading proteases, MMP-9 is known to degrade $A \beta$ fibrils in vitro, $A \beta$ plaques in 
situ, and A $\beta$ in vivo (Backstrom et al., 1996; Yan et al., 2006; Yin et al., 2006; HernandezGuillamon et al., 2015). Consequently, modulation of MMP-9 levels can therefore impact the clearance of $A \beta$ and promote its deposition.

Medroxyprogesterone Acetate (MPA) is a widely used, synthetic progestin that is the primarily found in the contraceptive, Depo Provera ${ }^{\circledR}$, and in HT, Prempro ${ }^{\circledR}$ and Premphase ${ }^{\circledR}$. Merlo et al., (2012) utilized estrogen to induce the A $\beta$-degrading activity of MMP-9, establishing MMP-2 and MMP-9's contribution to the neuroprotective effect of the hormone in vitro (Merlo and Sortino, 2012). Our work focused on MPA, and its ability to modulate the levels of MMP-9, and by extension the degradation of A $\beta$. MPA exerts adverse effects on cognition, substantiating the risk of dementia in menopausal women (Shumaker et al., 2003, 2004), promoting memory impairments in menopausal animal models (Braden et al., 2010, 2011, 2017), and inducing amnesia in case study reports of premenopausal women (Gabriel and Fahim et al., 2005). MMP-9 has been shown to be critical for cellular processes involved in learning and memory, as it regulates dendritic spine morphology, maintains late phase long term potentiation (LTP), and controls postnatal brain development (Nagy et al., 2006; Michaluk, 2010; Kamat et al., 2014; Gorkiewicz et al., 2015; Reinhard et al., 2015; Kaczmarek, 2016). MPA has also been shown to alter MMP-9 activity and production in BV2 microglial, cancer, and epithelial cells (Di Nezza et al., 2003; HwangLevine et al., 2011; Allen et al., 2019). Additionally, the secretion of MMP-9 was found to be negatively impacted by MPA in the aforementioned cell types (Deb and Gottschall, 1996; Deb et al., 2003; Hwang-Levine et al., 2011; Allen et al., 2019). It is currently unknown if hormonal modulation of glial-secreted MMP-9, using MPA, impacts degradation of A 3 . The 
pharmacological inhibition of MMP-2 and MMP-9, using both specific and non-specific enzymatic inhibitors, has also been shown to attenuate astroglia cell-mediated $A \beta$ degradation (Yin et al., 2006). Therefore, MPA-mediated reduction of glial-secreted of MMP-9 would likely result in dysregulated APP processing, fostering conditions which would disrupt clearance of $A \beta$. On the premises that: 1) this commonly used progestin negatively impacts the secretion of this $A \beta$-degrading enzyme, in the aforementioned cell types and 2) inhibition of glial secretion of MMP-9 reduces the proteolytic degradation of amyloid beta, our central hypothesis is that MPA diminishes the secretion of MMP-9, thereby reducing the degradation of amyloid beta.

Here, we provide evidence that illustrates the connection of MPA, MMP-9, and A $\beta$. We found that MPA influences $A \beta$ degradation by modulating the expression and/or activity of A $\beta$-degrading enzymes in a glial cell line (C6). C6 rat glial cells are a commonly used glial cell line and have also been identified as a useful cell line to study hormone action in glia (Kumar et al., 1986; Buchanan et al., 2000; Su et al., 2012b). We demonstrate that MPA significantly reduces enzymatic activity and secretion of MMP-9, and that MPA significantly reduces the degradation of $A \beta$. Together, these data implicate MPA in a negative effect on the A $\beta$-degrading enzyme, MMP-9. These data support a potential role where MPA perturbs $A \beta$ clearance mechanisms, indicating the necessity for in vivo investigations pertaining to MPA's influence on AD-related pathology.

\subsection{Materials and Methods}


Cell Culture. The C6 rat glial cell line was obtained from the American Type Culture Collection (ATCC \#CCL 107). C6 cells were maintained in Hyclone Dulbecco's modified Eagle's medium (DMEM)/high glucose (Fisher Scientific) supplemented with $10 \%$ fetal bovine serum (FBS; Atlanta Biologicals) and 1\% penicillin/streptomycin (Fisher Scientific). Once cells reached $80 \%$ confluency, they were trypsinized and spun down at $325.5 \mathrm{xg}$ for 3 min. Cells were counted with a Nexcelom Bioscience Cellometer AutoT4 (Lawrence) and seeded at a density of $1 \times 10^{6} \mathrm{cell} /$ well in 6 -well cluster plates, incubated overnight at $37^{\circ} \mathrm{C}$ in $5 \% \mathrm{CO}_{2}$.

Treatments. Serum-supplemented media was removed and the cell monolayer was washed once with phosphate buffer saline (1X PBS) and fresh-serum free media (OptiMEM; Fisher Scientific), containing either $0.1 \%$ dimethyl sulfoxide (DMSO; Millipore Sigma), various concentrations of Medroxyprogesterone Acetate (MPA; $10 \mathrm{nM}, 100 \mathrm{nM}, 1 \mu \mathrm{M}$, and $10 \mu \mathrm{M}$ ) (Millipore Sigma), GM6001 (100 $\mu \mathrm{M}$; Calbiochem, La Jolla, CA), or RU486 (2 $\mu \mathrm{M}$; Millipore Sigma). All treatments were diluted in serum-free medium to a final DMSO concentration of 0.1\%. Cell viability was always above $90 \%$ as assessed by Calcein AM (Fisher Scientific). C6 cells were incubated at $37^{\circ} \mathrm{C}$ in $5 \% \mathrm{CO}_{2}$ for 48 hours (48-h) or 72 -h. The conditioned media was collected and used to assess MMP-9 enzymatic activity (by gelatinase zymography), quantify extracellular MMP-9 (by ELISA), and assess proteolytic degradation of amyloid beta (by Western blot). Supernatants were stored at $-80^{\circ} \mathrm{C}$ and thawed on ice during experimental analyses. Repeated freeze thaw cycles were avoided by aliquoting the 
samples. The cells were collected and used to quantify intracellular MMP-9 protein levels (by ELISA) and assess MMP-9 mRNA expression (by qRT-PCR).

Cell viability. Cell viability was assessed using Calcein AM (Life Technologies) and reconstituted at $2 \mathrm{mM}$ in dimethylsulfoxide (DMSO). About 50,000 C6 cells were seeded in a black-walled clear bottom 96 well plate (Corning; \#3601). C6 cells were treated with 10 nM$10 \mu \mathrm{M}$ of MPA. After exposure to MPA $(10 \mathrm{nM}-10 \mu \mathrm{M})$ for $72-\mathrm{h}$, the plate was washed three times with PBS $1 \mathrm{X}$. A total of $100 \mu \mathrm{L}$ of $1 \mu \mathrm{M}$ Calcein AM was added to the wells. The plate was incubated at $22^{\circ} \mathrm{C}$ in the dark for $30 \mathrm{~min}$. The plate was read using a BioTek Synergy $\mathrm{H} 1$ Hybrid reader (Winooski, VT, USA).

Lactate Dehydrogenase Assay. Cell death after MPA treatment was assessed using the Pierce $^{\mathrm{TM}}$ lactate dehydrogenase (LDH) Cytotoxicity Assay (Fisher Scientific) kit. Reaction substrates were prepared as per the manufacturer's instructions. LDH assay was performed with the media from the black-walled clear bottom 96 well plate. Forty-five minutes prior to the end of the 72-h exposure period, $10 \mu \mathrm{L} 10 \times$ lysis buffer was added to one control well, and the plate was placed back in the incubator. At the conclusion of the exposure period, 50 $\mu \mathrm{L}$ of media was carefully removed from each well and transferred to a new 96 well clear bottom assay plate. Next, $50 \mu \mathrm{L}$ of the $\mathrm{LDH}$ reaction mixture was added to each well, and the plate was incubated for $30 \mathrm{~min}$ at room temperature, protected from light. The reaction was stopped by adding $50 \mu \mathrm{L}$ of LDH stop solution to each sample. The plate was read using the BioTek Synergy H1 Hybrid reader (BioTek) at absorbance of $490 \mathrm{~nm}$ and $680 \mathrm{~nm}$. 
Assay of MMP-9 activity by gelatin zymography. The gelatinolytic/proteolytic activity of MMP-9 secreted into the culture medium was determined with gelatin zymography by electrophoresis of serum-free conditioned media (CM) collected from confluent 66 cells, in accordance with Frankowski et al., (2012). Briefly, CM freed of cell debris by centrifugation were mixed with Laemmli sample buffer (Bio-Rad; \#1610747) lacking reducing agents. Electrophoresis in precast Novex polyacrylamide zymogram gels (Invitrogen) was performed at a constant voltage of $125 \mathrm{~V}$ for $90 \mathrm{~min}$ in SDS Tris-Glycine Buffer, followed by a series of three 15 minutes washes in $1 \mathrm{X}$ renaturation buffer (Invitrogen). The gels were transferred to $1 X$ Developing buffer (Life Technologies) for 30 minutes with gentle shaking and then placed at $37^{\circ} \mathrm{C}$ for an $18 \mathrm{~h}$ incubation. The following day, the gels were stained in a solution with $45 \%$ ethanol, $54 \%$ sterile $\mathrm{diH}_{2} \mathrm{O}, 1 \%$ acetic acid, and 0.125 g Coomassie brilliant blue R250 (Sigma) between 45 minutes to 1 - $h$. The gels were then transferred to de-staining solution \#1, containing $25 \%$ ethanol, $10 \%$ acetic acid, and $65 \% \mathrm{diH}_{2} \mathrm{O}$ for 45 minutes. Destaining solution \#1 was decanted and then replaced by a de-staining solution \#2, containing $5 \%$ ethanol, $7.5 \%$ acetic acid, and $87.5 \% \mathrm{diH}_{2} \mathrm{O}$ for $1-3 \mathrm{~h}$. Areas of gelatinolytic degradation appeared as transparent bands on the blue background. Gels were imaged using ChemiDoc ${ }^{\mathrm{TM}}$ XRS+ System (Bio-Rad, Hercules, CA). Images were acquired using BioRad Quantity One ${ }^{\mathrm{TM}}$ software. The Novex Sharp Pre-stained Protein Standard (Invitrogen) was used to identify MMP species or the MMP-9 Active, Human, Recombinant (Millipore Sigma; \#PF024-5UG) was used as a reference standard, showing MMP-9 gelatinolytic activity at $67 \mathrm{kDa}$. The bands in the gel are quantified using Image $1.38 \mathrm{X}(\mathrm{NIH})$. 
Intracellular and extracellular MMP-9 levels. CM samples and cell lysates were analyzed with a matrix metalloproteinase-9 (MMP-9) ELISA kit following the product manuals (R\&D Systems Quantikine ${ }^{\mathrm{TM}}$; \#RMP900). Results were calculated from the standard calibration curves on internal standards. After adding stop solution, optical density (OD) was measured at 450-nm with correction wavelength at 550-nm immediately using BioTek Synergy H1 Hybrid plate reader (BioTek). The final readings were obtained by subtracting $450-\mathrm{nm}$ from 550-nm OD reading to correct for optical imperfections of the microplate reader. A standard curve was generated with reagents provided in the kit and the sample values were read against the standard to determine MMP-9 concentrations in each treatment.

RNA isolation and Quantitative Real-Time PCR. Cells were seeded at $1 \times 10^{6}$ cells/well in a 6well plate and left to reach $~ 80 \%$ confluency over the course of 24-h. Media was gently aspirated from cells and replaced by $2 \mathrm{~mL}$ of OptiMEM Reduced Serum Media (Fisher Scientific), supplemented with 1\% penicillin/streptomycin (Fisher Scientific), or the various concentrations of MPA. After a 12-h exposure period, cells were collected using a Cell Lifter (Corning, Corning, NY, USA), washed with $1 \mathrm{X}$ PBS, and pelleted by centrifugation at $325.5 \times \mathrm{G}$ for $3 \mathrm{~min}$, followed by the addition of $1 \mathrm{~mL}$ QIAzol Lysis Reagent for RNA purification (Qiagen).

Total RNA was purified by using the miRNeasy Mini Kit (Qiagen) as per the manufacturer's instructions. For all lysate samples, $200 \mu \mathrm{L}$ chloroform was added, and vortexed for $1 \mathrm{~min}$ 
followed by incubation on Ice for 5-10 min. Next, the samples were centrifuged for $15 \mathrm{~min}$ at $12,000 \times \mathrm{g}$ at $4^{\circ} \mathrm{C}$. The upper aqueous phase $(\sim 300 \mu \mathrm{L})$ was transferred to a new collection tube and mixed with $600 \mu \mathrm{L} 100 \%$ ethanol. The solution was then placed on a RNeasy MinElute spin column and centrifuged at $8,000 \times G$ for $15 \mathrm{~s}$. The flow through was discarded and $700 \mu \mathrm{L}$ buffer RW1 (20\% Ethanol, $900 \mathrm{mM}$ guanidinium isothiocyanate (GITC), $10 \mathrm{mM}$ Tris- $\mathrm{HCl} \mathrm{pH} \mathrm{7.5)} \mathrm{was} \mathrm{added} \mathrm{to} \mathrm{the} \mathrm{spin} \mathrm{column} \mathrm{and} \mathrm{centrifuged} \mathrm{at} \mathrm{8,000 \times G} \mathrm{for} 15 \mathrm{~s}$. Two washes of $500 \mu \mathrm{L}$ buffer RPE (80\% Ethanol, $100 \mathrm{mM} \mathrm{NaCl}, 10 \mathrm{mM}$ Tris-HCl pH 7.5) were then performed, with the first lasting $15 \mathrm{~s}$ and the second lasting $2 \mathrm{~min}$. The RNeasy MinElute spin column was then placed in a new collection tube and spun at $8,000 \times 6$ for 5 min to dry the column membrane. Lastly, the column was placed in another collection tube and $40 \mu \mathrm{L}$ RNase-free water was added to the center of the membrane and incubated for $1 \mathrm{~min}$, and then centrifuged at $8,000 \times$ Gfor 1 min ending with the purified RNA eluted in the collection tube.

RNA concentrations for each sample were measured using Nano drop 2,000 spectrophotometer (Thermo Scientific). For cDNA synthesis, $0.75 \mu \mathrm{g}$ total RNA was reverse transcribed using the miScript II RT kit (Qiagen). A reaction mix (20 $\mu \mathrm{L}$ total volume) was made using $4 \mu \mathrm{L} 5 \times$ miScript HiFlexBuffer, $2 \mu \mathrm{L}$ 10× miScript Nucleics Mix, $2 \mu \mathrm{L}$ miScript Reverse Transcriptase Mix, and $12 \mu \mathrm{L}$ Template RNA/nuclease-free water. Prior to use for quantitative real-time PCR (qRT-PCR), cDNA was diluted in nuclease-free water, at a ratio of 1:10. 
Expression of MMP-9 mRNA was determined using target specific RT2 primer assays and the $\mathrm{RT}_{2} \mathrm{SYBR}^{\circledast}$ Green PCR kit (Qiagen; $5 \mu \mathrm{L}$ SYBR $^{\circledast}$ Green; $0.5 \mu \mathrm{L}$ Target Primer; $4.5 \mu \mathrm{L}$ diluted CDNA). qRT-PCR reactions were performed in duplicate for each sample, using the CFX384 TouchTM RT PCR Detection System (Bio-Rad) for 45 cycles as follows: $15 \mathrm{~s}$ at $94^{\circ} \mathrm{C}, 30 \mathrm{~s}$ at $55^{\circ} \mathrm{C}, 30 \mathrm{~s}$ at $70^{\circ} \mathrm{C}$. Negative control reactions were included as wells containing only master mix and nuclease-free water (no template cDNA). The expression levels of target genes in cell lysates was standardized against Adenylyl cyclase-associated protein 1(CAP-1) (IDT). Quantification of PCR amplified mRNA specific cDNA was done by comparative cycle threshold CT method (2- $\Delta \Delta C T)$. Ct values of mRNA were subtracted from the average $\mathrm{Ct}$ of the internal controls, and the resulting $\triangle \mathrm{CT}$ was used in the equation: relative copy numbers $=(2-\Delta \Delta C T)$.

Amyloid Beta preparation and amyloid beta degradation in astroglia-conditioned medium. Synthetic A $\beta 1-42$ (Invitrogen; \# 30112) was prepared from lyophilized A $\beta 1-42$ monomers that were suspended in $167 \mu \mathrm{L}$ of HPLC grade water (Fisher Scientific) and incubated at room temperature for $5 \mathrm{~min}$. The dissolved $\mathrm{A} \beta 1-42$ was then diluted to $230 \mu \mathrm{M}$ by adding $833 \mu \mathrm{L}$ of Ca2+-free phosphate buffered saline (PBS) and incubated for 48 -h at $37^{\circ} \mathrm{C}$ for polymerization. After polymerization, synthetic A $31-42(230 \mu \mathrm{M})$ at a final concentration of $23 \mu \mathrm{M}$ was freshly prepared and added to either serum-free media (SFM), untreated CM, MPA-treated CM, or GM6001-treated CM of C6 glial cells. The mixtures were then 
incubated at $37^{\circ} \mathrm{C}$ (Yin et al., 2006). After $24 \mathrm{~h}$, samples were collected and residual A $\beta 1-42$ was analyzed by Tris-Glycine-Western blotting.

Western blot was performed using denaturing 4-20\% Novex Wedgewell Tris-Glycine SDS gels (Invitrogen) with 90 min electrophoresis at 125V, 30 mA and iBlot Gel Transfer Gel Transfer Stacks (Invitrogen) with 7 min of electrical blotting. The polyvinylidene difluoride (PVDF) membrane (Invitrogen) with proteins transferred was blocked by Odyssey Blocking Buffer (LI-COR Biosciences; \#927-40000) for 1-h and was incubated with primary 6E10 (1:1000, Biolegend; \#803016) overnight and then with secondary (anti-mouse conjugated with fluorescence) (LI-COR) at room temperature for $90 \mathrm{~min}$. Bands were visualized using LICOR Odyssey IR Imager (LI-COR). Quantification using densitometric analysis was performed using Odyssey imaging systems (LI-COR biosystems, Lincoln, NE). Densitometry signal for each range of oligomers (low molecular weight aggregates (<15 kDa), intermediate-sized oligomers ( $15-55 \mathrm{kDa}$ ), high molecular weight oligomers (>56 kDa), or the entire lane for total $A \beta 1-42$ ) was normalized relative to the signal of the control lane (SFM) and fold change over untreated cells was plotted.

Statistical Analyses. All biological experiments were repeated at least three times with $\mathrm{n}=$ 3-16 plates/wells per treatment. Results from the experiments are reported as means \pm SEM. All quantitative data were assessed for significance using a one-way ANOVA with 
Dunnett's post hoc test. All results were analyzed by GraphPad Prism 8.0 software (GraphPad Software). A p value $<0.05$ was used to establish significance.

\subsection{Results}

\section{Medroxyprogesterone Acetate reduces MMP-9 enzymatic activity}

To study the effect of Medroxyprogesterone Acetate (MPA) on matrix metalloproteinase-9 (MMP-9) activity, we incubated C6 cells for 48 and 72-h with increasing concentrations of MPA (10 nM-10 $\mu \mathrm{M})$. The conditioned media (CM) were then collected and analyzed by gelatin zymography. This technique allows for the visualization of both active and proenzyme (inactive) forms of gelatinases (MMP-2 and MMP-9) (Frankowski et al., 2012). Untreated C6 cells show constitutive expression of active MMP-9 denoted by the $92 \mathrm{kDa}$ gelatinase band (Figure 1 A, B), with no detections of bands representative of MMP-2 activity. Densitometric analysis of zymograms obtained in 5 different experiments indicated that 48-h incubation of $\mathrm{C} 6$ cells with MPA inhibited MMP-9 activity in a dose-dependent manner, with significant reductions occurring at the three highest concentrations (100 nM, $1 \mu \mathrm{M}$, and $10 \mu \mathrm{M}$ ) compared to untreated control cells (Figure $1 \mathrm{C}, \mathrm{D})$. As expected, GM6001, an MMP inhibitor, suppressed MMP-9 enzymatic activity by $80 \%$. This dosedependent, inhibitory effect of both MPA and GM6001 on enzymatic activity persisted at 72-h. 
To determine whether the inhibitory effect of MPA on MMP-9 activity was due to interference with the activation process of MMP-9, post-secretion, we incubated $\mathrm{C} 6$ cells with medium alone. After 48-h, the CM were collected, divided in aliquots, and treated with increasing concentrations of MPA. Media was then incubated for 24 more hours at $37^{\circ} \mathrm{C}$ and analyzed by gelatin gel zymography. The inhibitory effect of MPA was lost, suggesting that MPA does not directly interact with MMP-9 and confirming the interference with the activity of the protease is cell-mediated (data not shown).

To exclude the possibility of drug toxicity as the reason of its inhibitory effect, C6 cells were incubated with increasing concentrations of MPA (10 nM, $100 \mathrm{nM}, 1 \mu \mathrm{M}$, and $10 \mu \mathrm{M})$ or 1 $\mu \mathrm{M}$ GM6001, then cell viability using Calcein AM and LDH release were assessed. MPA did not cause any appreciable cellular toxicity, even at the highest concentration used (10 $\mu \mathrm{M})$ (Supplementary Figure 1).

\section{Medroxyprogesterone Acetate reduces MMP-9 production}

Based on our finding that MPA caused decreased enzymatic activity of MMP-9, we next assessed whether the inhibitory effect of MPA on MMP-9 enzymatic activity was due to a reduction in overall secretion of MMP-9 protein or TIMP-1 inhibition. To measure extracellular and intracellular MMP-9 protein levels, C6 cells were incubated for 48-h with increasing concentrations of the drug. Both the media and the cell lysates were collected and analyzed by ELISA for MMP-9 (pro-MMP-9, TIMP bound MMP-9, and latent MMP-9) 
(Figure 2A, B). The analysis of three experimental replicates showed that MPA significantly inhibited extracellular MMP-9 protein levels (Figure 2A). MMP-9 inhibition ranged from 30\% to $60 \%$ relative to control (Figure $2 \mathrm{~A}$ ). Intracellular MMP-9 protein expression was also significantly decreased after MPA treatment, in a dose-dependent manner (Control:

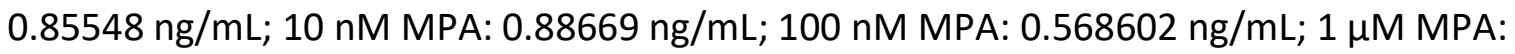
$0.468092 \mathrm{ng} / \mathrm{mL} ; 10 \mu \mathrm{M}$ MPA: $0.369965 \mathrm{ng} / \mathrm{mL}$ ) (Figure 2B). Because intracellular MMP-9 protein levels were negatively impacted by our MPA treatment, we evaluated the expression level of MMP-9 in cells treated with MPA by quantitative RT-PCR. Additionally, our zymography experiments revealed no detection of MMP-2 enzymatic activity, thus we performed an analysis of MMP-2 mRNA expression simply to confirm the lack of MMP-2 expression in the $\mathrm{C} 6$ cells (Figure 3A). Analysis of MMP-9 mRNA expression shows the experimental groups were significantly lower when compared with the control group (Figure 3A). Such repression appears after 12-h of treatment but is not detectable at later time points (24-h) (Figure 3B).

\section{Medroxyprogesterone Acetate's effects are mediated through the Glucocorticoid Receptor}

To investigate a potential mechanism by which MPA causes repression of MMP-9 transcription, we first considered a receptor-mediated approach. At the molecular level, MPA elicits its biological effects through multiple receptors, including the progesterone receptor (PR), androgen receptor (AR), and glucocorticoid receptor (GR) (Africander et al., 
2011). Notably, these cells are devoid of the PR and AR (Su et al., 2012b). The GR, however, is present in C6 cells and MPA binds to the GR with a high affinity, acting as a partial to full agonist for the GR (Koubovec et al., 2004, 2005; Su et al., 2012b, 2012a; Louw-du Toit et al., 2014). We tested the hypothesis that MPA's effects on enzymatic activity and production of MMP-9 are mediated by the GR (Figure 4). C6 cells were pretreated with mifepristone, RU486 ( $2 \mu \mathrm{M})$, for $30 \mathrm{~min}$, followed by MPA treatment for another 48-h, using the lowest and highest concentrations of MPA at which significant effects were seen on enzymatic activity, intracellular protein, and mRNA levels (100 nM and $10 \mu \mathrm{M})$. Densitometric analysis of the zymogram (Figure 4A) shows pharmacological inhibition of the GR with RU486 attenuated MPA's effect on MMP-9 activity (100 nM MPA: 51.61\%; 100 nM MPA/RU486: 82.23\%; $10 \mu \mathrm{M}:$ 41.99\%; $10 \mu \mathrm{M}$ MPA/RU486: 64.79\% (Figure 4B). This suggests our findings are potentially due, in part, to a GR-mediated mechanism.

\section{Medroxyprogesterone Acetate antagonizes the degradation of $A \beta$}

Previous reports have suggested that MMP-9 is capable of degrading amyloid beta, in vitro (Backstrom et al., 1996). To explore the possibility that inhibition of MMP-9 would interfere with amyloid beta degradation, $\mathrm{C} 6$ wells were treated with MPA for 48-h and the CM were incubated with freshly prepared synthetic human $A \beta_{1-42}$ for $24-h$ at $37^{\circ} \mathrm{C}$. The doses selected for this experiment were the lowest and the highest dose at which we observed significant effects on enzymatic activity, intracellular protein, and mRNA levels (100 nM and $10 \mu \mathrm{M})$. Using the anti-A $\beta 6 \mathrm{E} 10$ antibody, $A \beta$ levels were then measured by Tris-Glycine Gel-Western 
blotting (Figure 5A). This approach yielded the resolution of groups of oligomers as indicated in Figure 5A, consistent with the literature (Prangkio et al., 2012). Incubation of $A \beta$ with $C M$ resulted in a significant reduction in $A \beta$ levels, causing the appearance of several A $\beta$ fragments, which are indicative of MMP cleavage (Backstrom et al., 1996). This A $\beta$-degrading activity was antagonized by MPA (100 nM and $10 \mu \mathrm{M})$. Densitometric analysis (Figure 5B-E) established that CM modestly altered low molecular weight aggregates $(<15$ kDa), while MPA-treated CM samples showed no effect (Figure 5B). However, MPA-treated CM samples overall mitigated proteolytic cleavage of intermediate-sized oligomers ( $\sim 15-55$ $\mathrm{kDa}$ ) (Figure 5C), high molecular weight oligomers (>56 kDa) (Figure 5D), and total (Figure 5E) $A \beta$ species. Incubation of $A \beta_{1-42}$ with $C M$ significantly abated $A \beta$ levels and MPA treatment weakened this effect. To verify the contribution of MMPs to $A \beta$ degradation in $C M$, we incubated $C M$ with freshly prepared synthetic human $A \beta_{1-42}$ for $24-h$ at $37^{\circ} \mathrm{C}$ in the presence or absence of the broad-spectrum, MMP inhibitor, GM6001, and found that the A $\beta$-degrading activity in CM was attenuated (Supplemental Figure 2). Additionally, we incubated SFM with freshly prepare synthetic human $A \beta_{1-42}$ for $24-h$ at $37^{\circ} \mathrm{C}$ in the presence or absence of active recombinant MMP-9 protein (rMMP-9). These data confirmed findings in the literature that MMP-9 possesses A $\beta$-degrading activity (data not shown) (Backstrom et al., 1996; Yan et al., 2006; Hernandez-Guillamon et al., 2015). Next, we assessed the possibility of a direct action of MPA on A $\beta$. We treated SFM with MPA for 48-h. incubated the supernatant with $23 \mu \mathrm{M} \mathrm{A} \beta_{1-42}$, for an additional $24-\mathrm{h}$ at $37^{\circ} \mathrm{C}$, and we found MPAtreated SFM failed to digest $A \beta$ (data not shown). We determined our observed impairment of proteolytic degradation occurred by an indirect, cell-mediated mechanism. 


\subsection{Discussion}

Ninety percent of the cells in the central nervous system (CNS) are glia (Haydon, 2001). Glial cells (astrocytes, oligodendrocytes and microglia) communicate with neurons to regulate synaptic plasticity and neurotransmission (Fields et al., 2014). Accumulating evidence suggests MMP secretion from astroglia contribute to the degradation and clearance of amyloid plaques (Yan et al., 2006; Yin et al., 2006; Wang et al., 2014), emphasizing the critical role of induction and secretion of MMPs in the brain. There are several reports showing astroglia conditioned media (CM) possesses $A \beta$-degrading activity, in part, through the secretion of MMPs (Backstrom et al., 1996; Yin et al., 2006; Fragkouli et al., 2014). Moreover, MMP-9 possesses $\alpha$-secretase-like activity and cleaves APP at several sites, promoting the non-amyloidogenic processing of the precursor protein and clearance of $A \beta$ (Backstrom et al., 1996; Yin et al., 2006; Talamagas et al., 2007; Filippov and Dityatev, 2012).

The present study investigated the expression of MMP-9 in C6 glial cells treated with MPA. Several reports have demonstrated that MPA can alter MMP-9 levels, where levels are increased in macrophages and, alternatively, reduced in BV2 microglial, endometrial cancer, and primary amnion epithelial cells (Di Nezza et al., 2003; Hwang-Levine et al., 2011; Allen et al., 2019). Consistent with these reports, we found that MMP-9 protein expression and enzymatic activity is lowered by treatment with MPA. However, majority of the previous in vitro studies have used non-CNS cell lines to observe the effect of MPA on MMP-9, with 
little to no investigation of the effects of MPA on glial secretion of MMP-9. Additionally, due to the lack of focus in a CNS-related system, previous work failed to investigate the potential for MPA to influence AD-related pathology via the hormonal modulation of MMP9.

To our knowledge, this is the first in vitro analysis of the effect of MPA on MMP-9's enzymatic activity and production, in a $\mathrm{C} 6$ glial cell line. Our results show that MMP-9 is expressed in C6 glial cells in control conditions and is suppressed by exposure to MPA at both the mRNA and the protein level. We found that MPA suppresses MMP-9 activity in a dose-dependent manner (Figure 1). Decreased MMP-9 activity was reflected by impaired synthesis of the MMP-9 protein (Figure 3A, B) and was further supported with data showing a suppression of MMP-9 mRNA (Figure 3A). We observed a spurious increase at the highest concentration of MPA (10 $\mu \mathrm{M})$, which may be indicative of off-target effects because of such a high dose of the hormone. In agreement with our hypothesis, experiments on CM confirmed the inhibitory effects of MPA on the enzymatic activity of MMP-9. Conversely, we found MPA did not cause secretory inhibition of MMP-9, but rather suppressed the transcription of MMP-9 at the mRNA level.

The glucocorticoid receptor (GR) is typically found, in an inactive state, in the cytoplasm, and, upon ligand binding, it becomes activated and transrepresses pro-inflammatory genes. This is thought to be substantiated either through direct DNA-binding, by binding a 
glucocorticoid response element (GRE) or the nuclear factor kappa B (NF-KB) response element (KBRE) (Meijsing et al., 2009; Surjit et al., 2011; Watson et al., 2013; Weikum et al., 2017; Sacta et al., 2018; Hudson et al., 2018) or 2), or through a DNA independent, direct protein-protein interaction/crosstalk with other transcription factors, including NF-KB and AP-1 (McEwan et al., 1997; Webster and Cidlowski, 1999; De Bosscher et al., 2003; Liu and Xu, 2012; Trevor and Deshane, 2014). The initial finding of MPA's capability of interfering with the activities of NF-KB or AP-1, at the promoter level, suggested MPA represses cytokine-induced, AP-1 driven genes, as well as NF-KB-driven genes, without impacting the DNA-binding activity of NF-KB, in a GR-dependent manner (Koubovec et al., 2004). Conversely, Simoncini et al., (2004) demonstrated MPA's anti-inflammatory effects and MPA's ability to reduce hydrocortisone-dependent nuclear translocation of NF-KB in human endothelial cells. $\mathrm{C} 6$ cells have spontaneous NF-KB nuclear activity, suggesting the presence of constitutive NF-KB activity, which explains the constitutive basal expression of MMP-9 observed in our experiments (Robe et al., 2004). We treated C6 cells with a GR antagonist, which attenuated MPA's effects on MMP-9 enzymatic activity (Figure 4). In this case, we suspect there may be a GR-dependent transrepression of MMP-9, via interaction with either NF-KB or AP-1, which are generally accepted as regulators of MMP-9 expression (Jonat et al., 1990; Paliogianni et al., 1993; Yokoo and Kitamura, 1996; Barnes, 1998; Bond et al., 2001; Ronacher et al., 2009; Africander et al., 2011; Li et al., 2012; Mittelstadt and Patel, 2012). The literature also extensively supports the notion that glucocorticoids alter MMP-9 expression (Rosenberg et al., 1996; Park et al., 1999; Eberhardt et al., 2002; de Paiva et al., 2006), as well as MMP-3 (Richardson and Dodge et al., 2003; Koyama et al., 2017), 
which is the enzyme responsible for the conversion of MMP-9 from an inactive to an active state. Therefore, it's plausible the progestin binds to the cytosolic $G R$, activates the $G R$, and then activated GR binds to NF-KB, in turn, negatively interfering with the transcriptional enhancer and, in turn, promoting transrepression of MMP-9. Notably, C6 cells have also been shown to express mRNA for the membrane progesterone receptors (mPR) (Su et al., 2012a). Salazar et al., (2016) demonstrated MPA elicits progestin-induced intracellular signaling in PR-negative breast epithelial cells, suggesting a potential mode of action via membrane progesterone receptors. It is possible that our observations may be partially due to non-genomic signaling through membrane progesterone receptors, however, there is currently a lack of information regarding MPA's relative binding affinity to membrane progesterone receptors and a lack of evidence relating to MPA's propensity to elicit its effects via non-genomic membrane bound signaling. Future studies should aim to further delineate the precise mechanisms by which our observations in the present study occur.

To our knowledge, this is the first investigation of the effects of MPA treatment on MMP-9's $A \beta$-degrading activity. Our objective was to relate progestin-induced reductions of active MMP-9 levels with a loss of $A \beta_{1-42}$ degradation. We were interested in observing the effects after exposure to high concentrations of $A \beta(23 \mu \mathrm{M})$ and, using Western blot analysis, we were able to confirm the diminished ability of MPA-treated C6 cells to degrade $A \beta_{1-42}$ (Figure 5). A key finding of our current study was that MPA-treated CM samples did not significantly alter low molecular weight aggregates (Figure 5B). However, MPA treatment impaired the degradation of intermediate-sized and high molecular weight oligomeric $A \beta$ 
species.. Of interest is the observed effect on oligomeric $A \beta$, which is suggested to be the culprit of the neurodegeneration seen in AD (Lesné et al., 2008, 2013; Lublin and Gandy, 2010). Our data suggests MPA treatment potentially promotes AD-related pathology. Our inclusion of the non-specific MMP inhibitor, GM6001, yielded a greater effect on degrading activity. This affirmed our observations were, in part, MMP-9 dependent, as GM6001 was shown to inhibit the enzymatic activity of MMP-9 by $80 \%$ (Figure 1 ). Enzymatic activity of MMP-9 was inhibited to the same extent by GM6001 and $10 \mu \mathrm{M}$ MPA, which was reflected in their hindrance of $A \beta$ degradation. Degradation of $A \beta$ was not fully thwarted with GM6001 treatment, implicating the potential for additional proteases that exist in CM. As previously discussed, in addition to MMPs, $A \beta$ can be degraded by other proteases, including ECE, IDE, and NEP (Saido and Leissring, 2012). Prior literature supports a minimal contribution of IDE, NEP, and ECE as extracellular, secreted amyloid beta scavengers from astrocytes. According to Yin et al., (2006), in addition to NEP, ECE, and IDE, there may be proteases that have yet to be experimentally identified, contributing to astrocyte-mediated degradation of $A \beta$, due to the fact that NEP, ECE, and IDE were undetected with Western blot analysis of conditioned media of neonatal mouse astrocytes. Moreover, it has been reported that cultured cells may be incapable of secreting IDE, and it is still undetermined whether NEP is capable of degrading oligomeric amyloid beta (Saido et al., 2012; Song et al., 2018). There is evidence that estrogen promotes amyloid beta degradation through the induction of NEP (Liang et al. 2010), and there is also evidence that IDE is induced by $17 \beta$ estradiol, reducing amyloid beta load in vivo (Zhao et al. 2011). Thus, the literature generally supports the idea that hormones are capable of regulating these specific amyloid 
beta degrading enzymes and suggests MPA could also potentially regulate additional amyloid beta degrading enzymes. However, we focused on MMP-9, because MMP-9 is the only amyloid beta scavenger shown to possess the ability to degrade $A \beta$ fibrils in vitro, $A \beta$ plaques in situ, and $A \beta$ in vivo, making it the most unique and distinguished of the known scavengers (Backstrom et al, 1996; Yan et al, 2006; Yin et al., 2006; Hernandez-Guillamon et al., 2015). Overall, our findings suggest MMPs are involved in the degradation of $A \beta$ in $C M$ and MPA impedes on the degradation, through the downregulation of MMP-9 production, in a GR-dependent manner.

We noted several limitations in the present study. First, our synthetic $A \beta$ formulation and its aggregated forms may not fully represent the in vivo phenomena, due to variability in oligomer generation (refer to Supplementary Figure 8). Additionally, the effective concentrations of MPA in our study ranged from $10 \mathrm{nM}$ to $10 \mu \mathrm{M}$. Although our working concentrations are relatively high, peak serum concentrations of MPA fall between $10 \mathrm{nM}$ and 100 nM (Tomasicchio et al., 2013), after women receive an intramuscular injection of $150 \mathrm{mg}$, every three months. These are concentrations at which our observed effects on MMP-9 and A $\beta$-degrading activity occurred. Furthermore, our in vitro model utilizes a transformed glial cell line. The utilization of primary cells are more reliable than cell lines, however, this would require pharmacological induction of MMP-9 expression or transfection of an MMP-9 expression vector. As previously mentioned, C6 cells have spontaneous NF-kB nuclear activity, suggesting the presence of constitutive NF-KB activity, which explains the constitutive basal expression of MMP-9 observed in our experiments 
(Robe et al., 2004), and circumvented the necessity for induction or transfection of MMP-9 expression. Although in vitro experiments using cell culture are useful, providing pertinent information, ultimately in vivo experiments are needed to confirm these effects.

Based on our findings, progestin-induced downregulation of MMPs is partially responsible for hampering the proteolytic cleavage of $A \beta_{1-42}$ and supports a possible link between MPA administration and AD-related pathology. The importance of MMP-mediated degradation of toxic $A \beta_{1-42}$ species and its potential neuroprotective effect is abolished with MPA-induced reduction in MMP-9 expression and production. Most importantly, this commonly used progestin suppresses both the transcription and the activation of glial MMP-9, which is responsible for reduced $A \beta$ degradation. Taken together, our study confirmed $A \beta$-degrading activity was reduced, in part, through the impairment of MMP-9 production.

In summary, our work demonstrates the necessity for a further delineation of MPA's effects on MMP-9 production. We also raise awareness for the unmet need of in vivo investigation for the potential cognitive and pathological outcomes of MPA. There is currently an abundance of literature which focuses on the effects of estrogen, estradiol, testosterone and progesterone on the brain, and particularly amyloid beta production and clearance (Vest et al., 2013; Li et al., 2014; Giatti et al., 2016; Uchoa et al., 2016). There is still a paucity of published research addressing the potential effects of progestins, more specifically MPA, on the brain. While limited research with MPA suggests a negative impact 
on the brain, these studies need to be extended to model systems relevant to women in their reproductive prime, as majority of efforts have been in menopausal animal models and menopausal woman (Shumaker et al., 2003, 2004; Braden et al., 2010, 2011, 2017; Lowry et al., 2010; Olanrewaju et al., 2013). Our finding that MPA limits MMP-9 production could ultimately negatively impact synaptic plasticity in vivo, as MMP-9 has been shown to be considered "indispensable" for neuronal plasticity (Nagy et al., 2006; Michaluk et al., 2011; Kamat et al., 2014; Gorkiewicz et al., 2015; Lepeta and Kaczmarek, 2015; Kaczmarek, 2016). Thus, independent of AD-related pathology, MPA's suppression of MMP-9 production could still prove harmful for learning and memory. Herein, we encourage additional investigations related to MPA's effects on the brain. Future efforts should be extended to model systems relevant to AD-related pathology. It is conceivable that the prolonged use of MPA will progressively subdue the proteolytic degradation of A $\beta$ by MMP9, in vivo, promoting AD-related pathology. This hypothesis is currently being tested in our laboratories.

\section{Acknowledgments}

This work was supported by the NIH grants P20 GM109098, P01 AG027956, and U54 GM104942.

Author Contributions

KNP designed studies, conducted studies, and composed manuscript. SNS, DAD, MEV, and DDQ aided with studies and revised the manuscript. JWS designed studies and revised the manuscript.

Conflict of Interest Statement

The authors declare that the research was conducted in the absence of any commercial or financial relationships that could be construed as a potential conflict of interest. 


\subsection{Figures}

A $\quad$ B

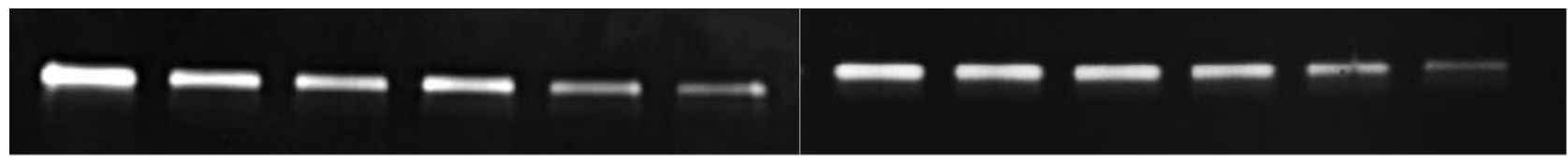

C

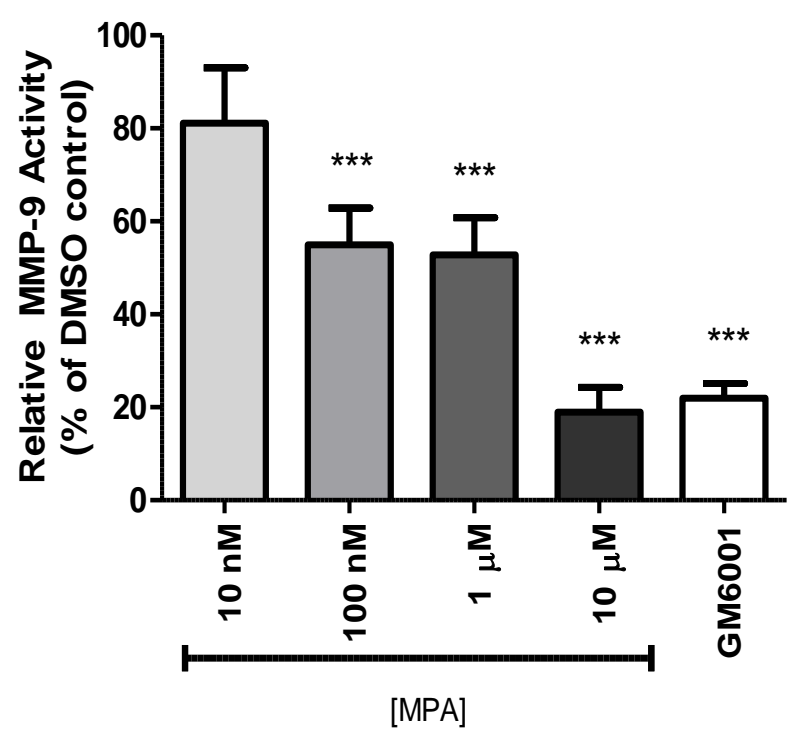

D

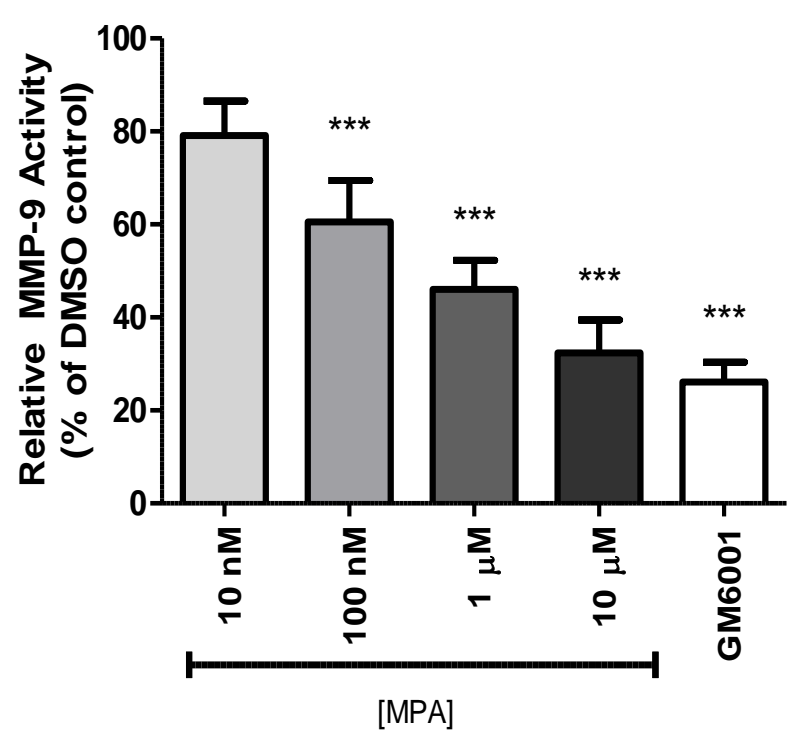

Figure 1. MPA reduced the gelatinolytic activity of MMP-9 in C6 glial cells. (A, B) Representative zymograms showing MMP-9 activity of MPA-treated conditioned media (CM) determined by gelatin zymography. After a 48-h (left) or 72-h (right) incubation period in serum-free media, supernatants obtained from $1 \times 106$ cells were analyzed by gelatin zymography. DMSO at $0.1 \%$ was used as the vehicle control and constitutively showed MMP-9 gelatinolytic activity (lane 1). Upon MPA treatment, MMP-9 gelatinolytic activity was significantly decreased (lanes 2-5). GM6001 (lane 6) was used as a negative control for active MMP-9. (C, D) Densitometric analysis of conditioned media from astroglia determined by gelatin zymography. $\mathrm{C} 6$ glial cells were incubated for 48-h (left) or 72-h (right) with increasing concentrations of MPA and GM6001, a non-specific MMP inhibitor. Results are expressed as percentage of activity of treated to untreated cells (mean \pm SEM). MMP-9 gelatinolytic activity of untreated cells is expressed as $100 \%$. MPA significantly decreased MMP-9 enzymatic activity in a dose-dependent manner $(P<0.001)$ compared with that found in untreated cells. Results are representative of five independent experiments. $* * * p<0.001$. 
A

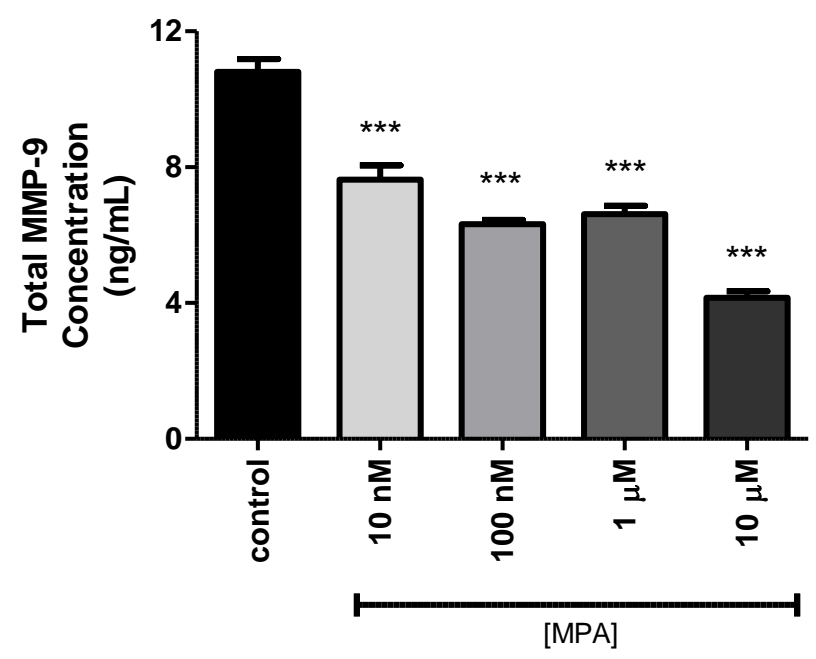

B

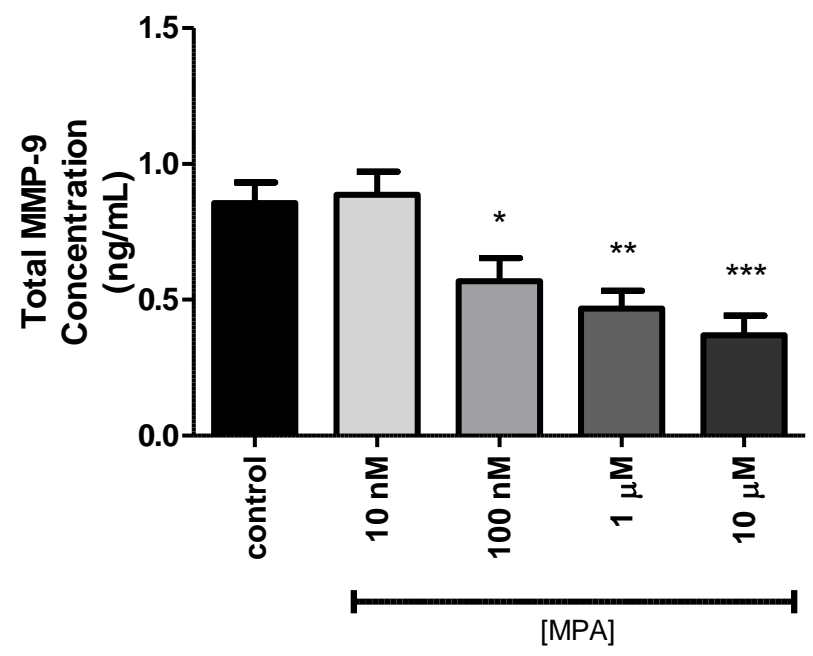

Figure 2. The effect of 48-h MPA treatment on MMP-9 protein in C6 glial cells. (A) Extracellular MMP-9 secretion was detected using ELISA kit. MPA inhibited the expression of extracellular MMP-9 in treated $\mathrm{C} 6$ glial cells. Results are representative of three independent experiments. (B) The effect of 48-h MPA treatment on intracellular MMP-9 in C6 glial cells. MMP-9 secretion was detected using ELISA kit. Results are expressed as means \pm SEM. MPA inhibited the expression of intracellular MMP9 in treated C6 glial cells. Results are representative of five independent experiments. ${ }^{*} p<0.05,{ }^{* *} p$ $<0.01$, and ${ }^{* * *} p<0.001$. 
A

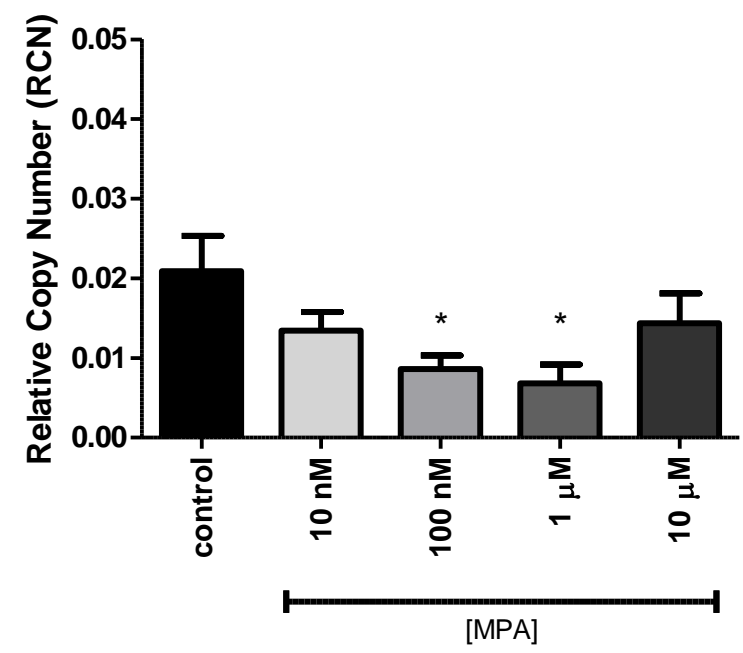

B

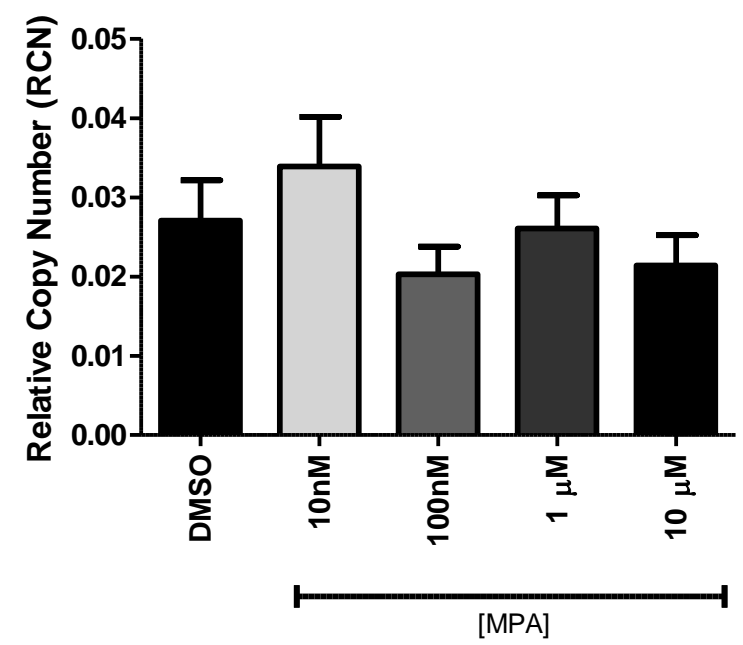

Figure 3. The effect of MPA treatment on MMP-2/-9 mRNA in C6 glial cells. (A) The mRNA levels of MMP-2 and MMP-9 were measured by qRT-PCR in C6 glial cells treated with MPA for $12-\mathrm{h}$, using MMP-2 and MMP-9 specific primers. (B) At 24-h, the suppression of MMP-9 mRNA levels is no longer detected. Data are represented by mean \pm SEM of 3 independent qRT-PCR experiments performed in duplicates. The expression levels are represented relative to GAPDH reference gene. Results are representative of five independent experiments. ${ }^{*} p<0.05$ and ${ }^{* *} p<0.01$. 

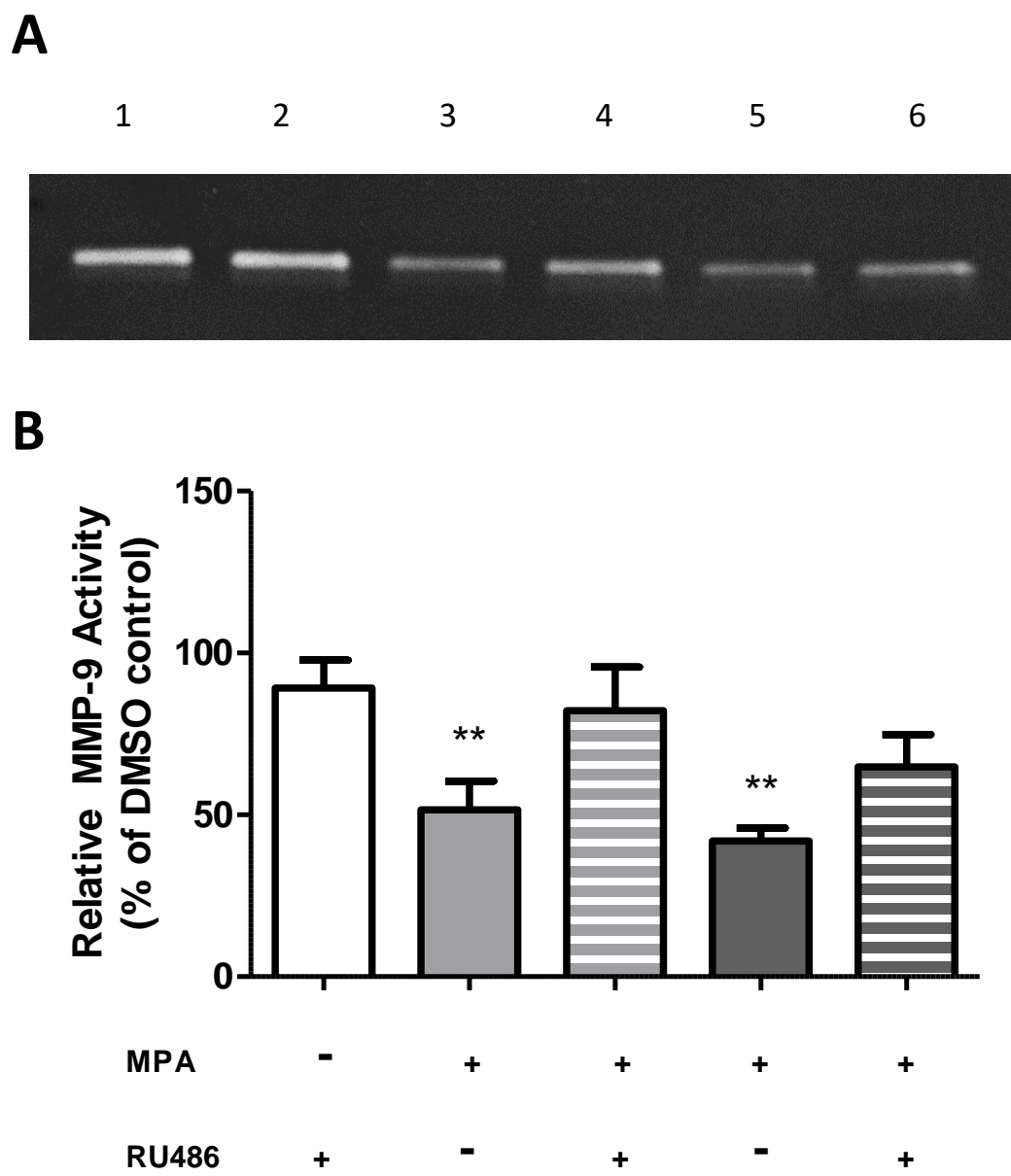

Figure 4. RU486 pretreatment antagonized the effects of MPA on MMP-9 activity. (A) Representative zymogram showing MMP-9 activity of MPA-treated conditioned media (CM). DMSO at $0.1 \%$ was used as the vehicle control and constitutively showed MMP-9 gelatinolytic activity (lane 1). $C 6$ cells were pretreated with mifepristone, RU486 ( $2 \mu \mathrm{M})$, for $30 \mathrm{~min}$, followed by MPA treatment for another 48-h. Treatment with RU486, alone, did not alter MMP-9 enzymatic activity (lane 2). Upon MPA treatment, MMP-9 gelatinolytic activity was significantly decreased (100 nM, lane 3; $10 \mu \mathrm{M}$, lane 5). Pretreatment with RU486 (2 $\mu \mathrm{M})$ antagonized MPA's effect on enzymatic activity (100 nM, lane 4; $10 \mu \mathrm{M}$, lane 6). (B) Densitometric analysis of conditioned media from astroglia determined by gelatin zymography. Results are expressed as percentage of activity of treated to untreated cells (mean \pm SEM). MMP-9 gelatinolytic activity of untreated cells is expressed as $100 \%$. RU486 treatment is represented by the white bar, $100 \mathrm{nM}$ MPA treatments are represented by the light grey bars (solid and striped), $10 \mu \mathrm{M}$ MPA treatments are represented by the dark grey bars (solid and striped). Results are representative of three independent experiments. $* * p<0.01$. 
A

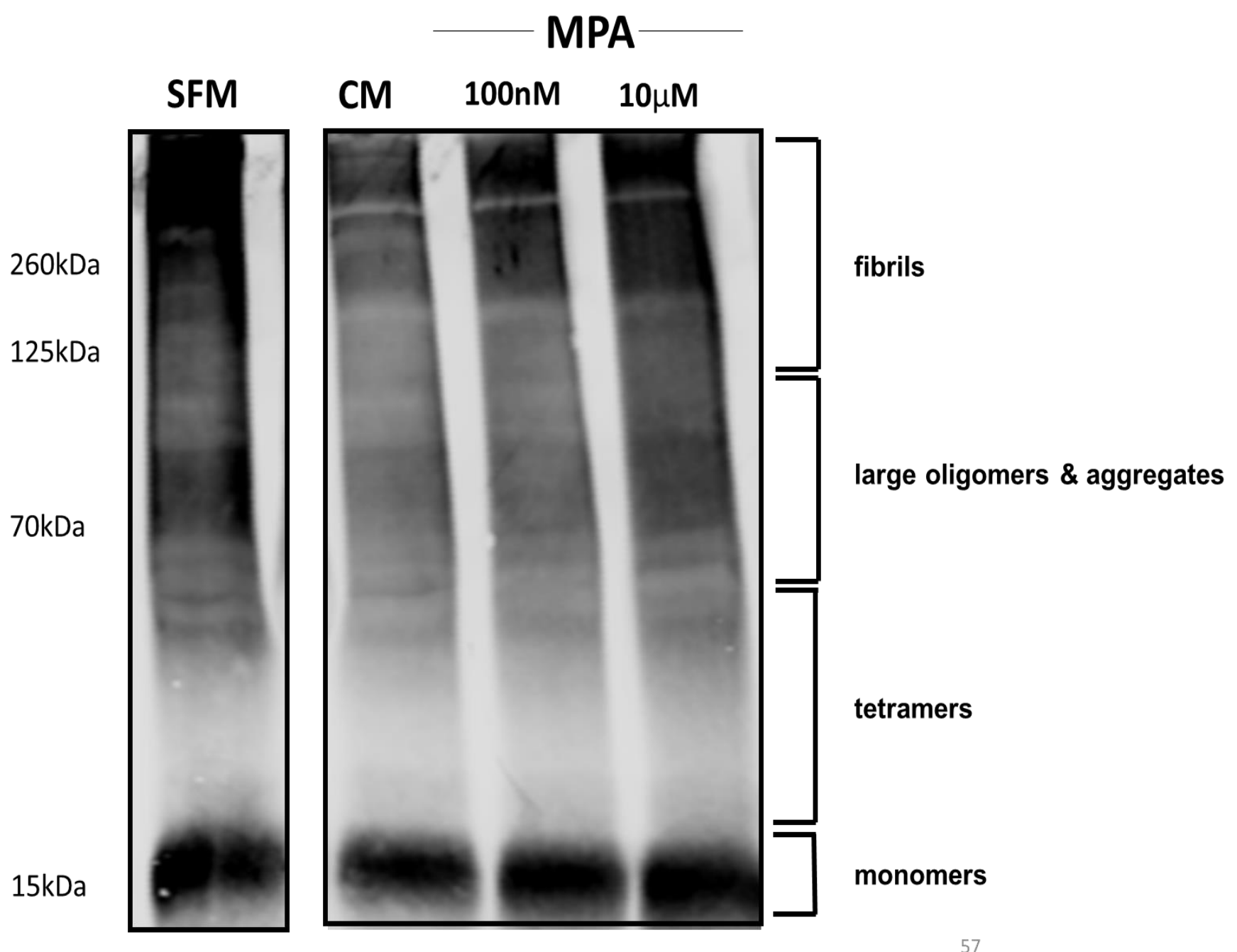


B

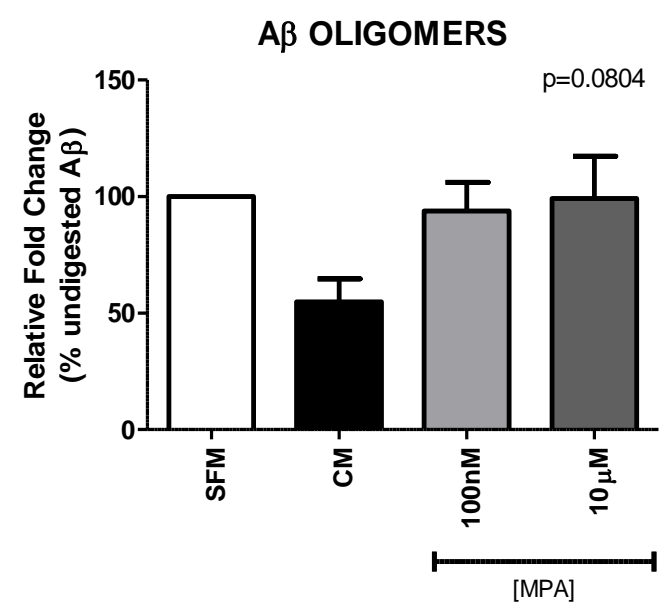

D

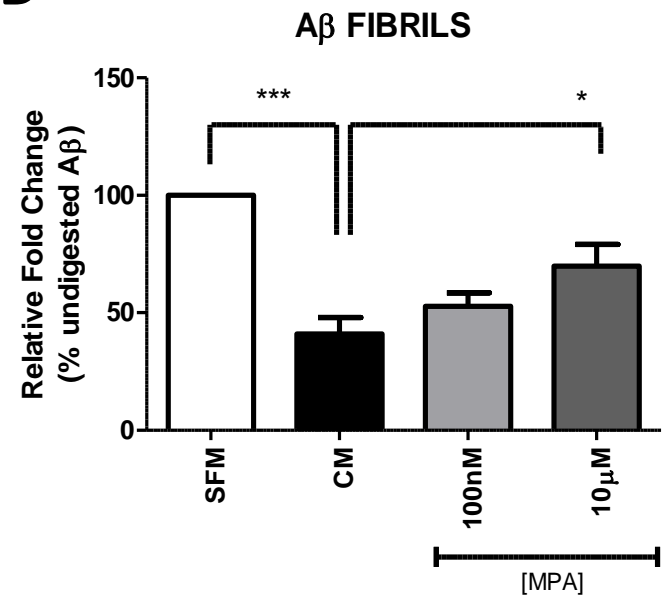

C

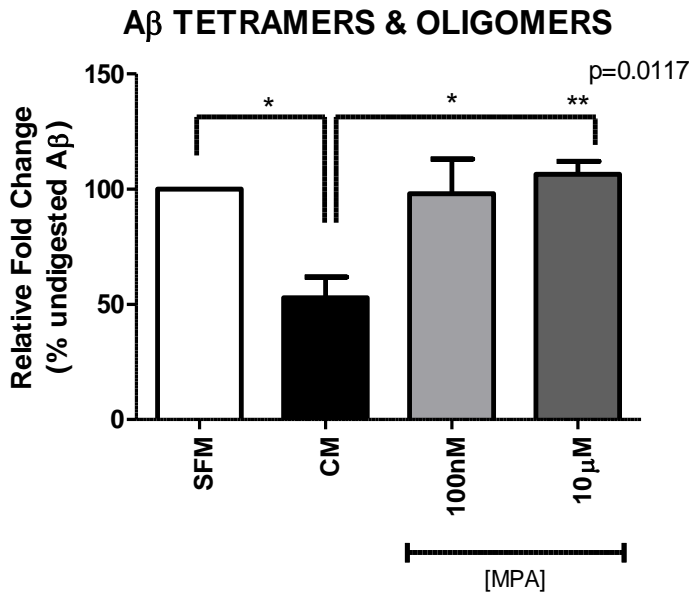

$\mathbf{E}$

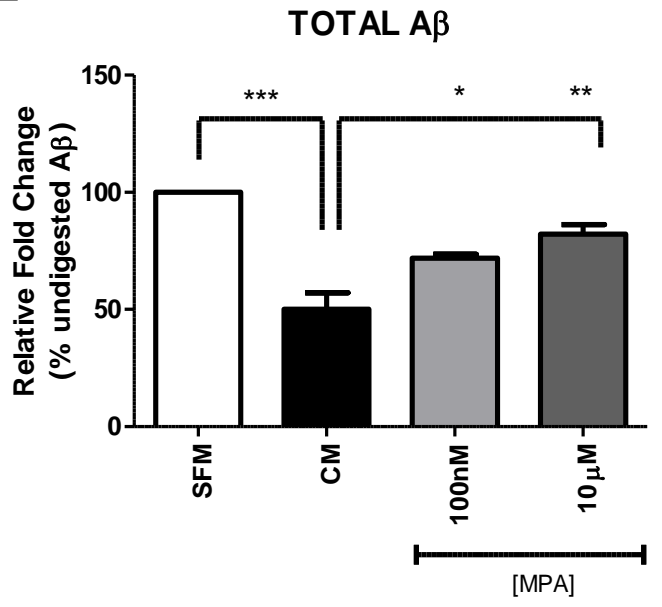

Figure 5. Western blot detection and densitometric analysis of $A \beta$ using $6 E 10$ antibody (A) Representative Western blot of $A \beta$ using $6 E 10$ antibody. Freshly prepared synthetic human $A \beta 1-42$ $(23 \mu \mathrm{M})$ was added to serum-free media (SFM) (lane 1), SFM that had been conditioned by incubation with $\mathrm{C} 6$ glial cells (CM) (lane 2), or CM that had been treated with MPA (100 nM or 10 $\mu \mathrm{M}$ ) for 48-h (lanes 3 and 4). The mixture was then incubated for $24 \mathrm{~h}$ at $37^{\circ} \mathrm{C}$, and residual $A \beta$ was analyzed by Tris-Glycine-Western blotting. Incubation of $A \beta_{1-42}$ with $C M$ significantly decreased $A \beta$ levels. MPA treatment attenuated this effect. (B-E) Densitometric analysis shows that MPA-treated $\mathrm{CM}$ samples induced significant differences in $A \beta$ species. Incubation of $A \beta 1-42$ with $C M$ significantly decreased $A \beta$ levels. MPA treatment attenuated this effect. Results are representative of three independent experiments. ${ }^{*} p<0.05,{ }^{* *} p<0.01$, and ${ }^{* * *} p<0.001$. 
A

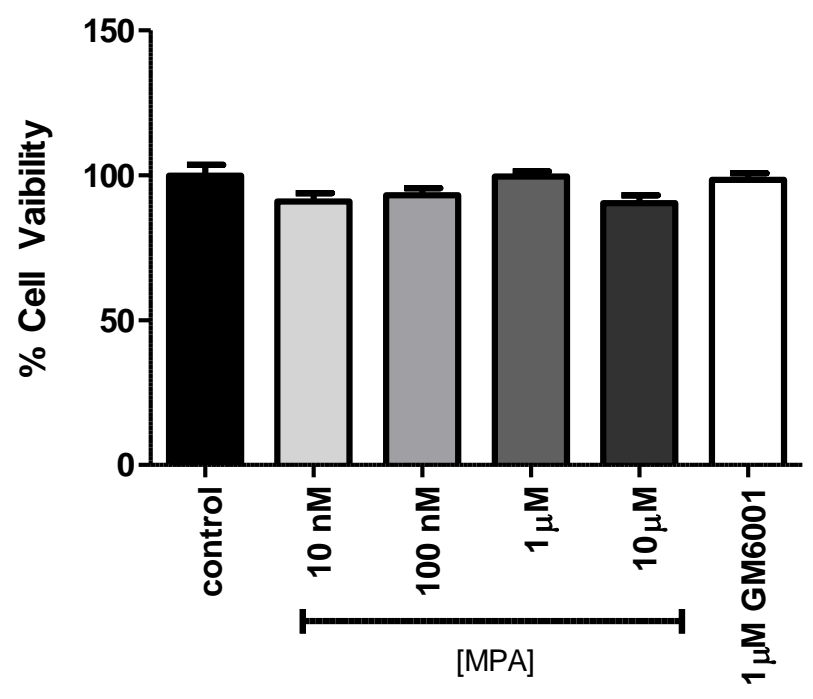

B

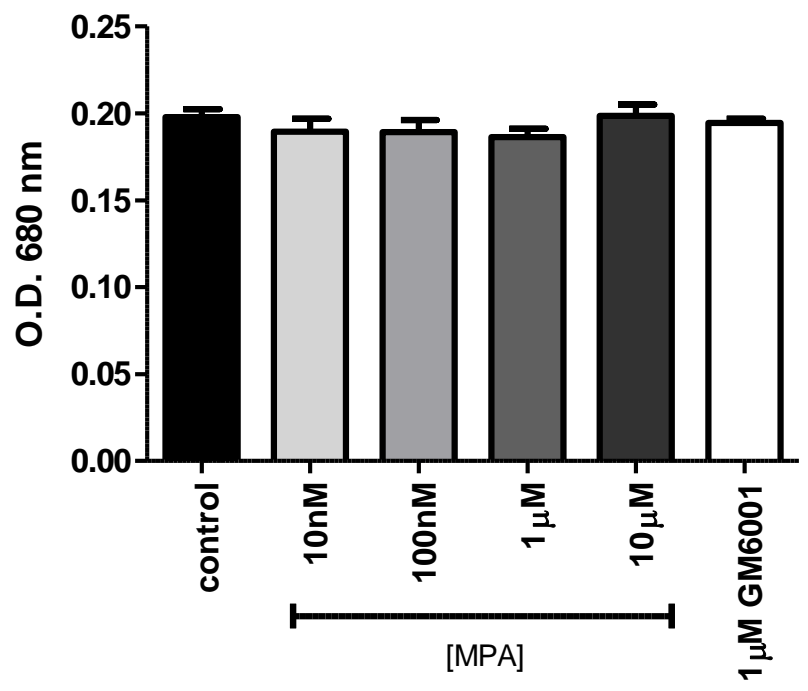

Supplementary Figure 1. Effect of MPA on cell viability and LDH release. (A) Calcein AM and (B) Lactate dehydrogenase (LDH) data indicate that significant cell death did not occur at any concentration of MPA treatment. 
A

\section{SFM CM GM6001}

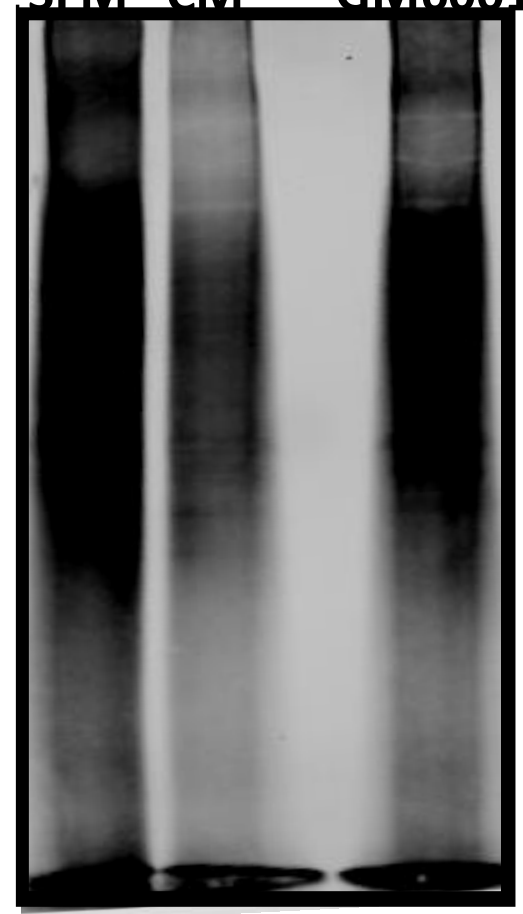

B

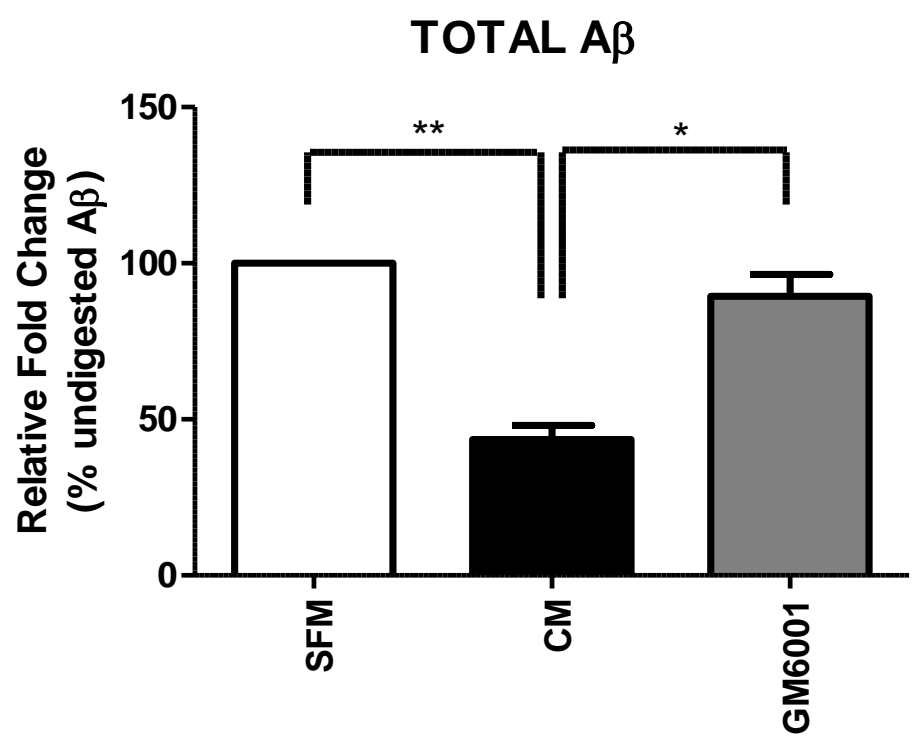

Supplementary Figure 2. Western blot detection and densitometric analysis of A $\beta$ using 6E10 antibody. Representative Western blot of $A \beta$ using $6 \mathrm{E} 10$ antibody. Freshly prepared synthetic human AB1-42 was added to serum-free media (SFM) (lane 1), SFM that had been conditioned by incubation with $\mathrm{C} 6$ glial cells (CM) (lane 2), or CM that had been treated with $1 \mu \mathrm{M}$ GM6001 (lane 3) for 48 - $h$. The mixture was then incubated for $24 \mathrm{~h}$ at $37^{\circ} \mathrm{C}$, and residual $A \beta$ was analyzed by (A), TrisGlycine-Western blotting. (B) Densitometric analysis of overall total $A \beta 1-42$ shows that incubation of $A \beta 1-42$ with CM significantly decreased $A \beta$ levels and GM6001 treatment attenuated this effect. Results are representative of two independent experiments. ${ }^{*} p<0.05,{ }^{* *} p<0.01$. 


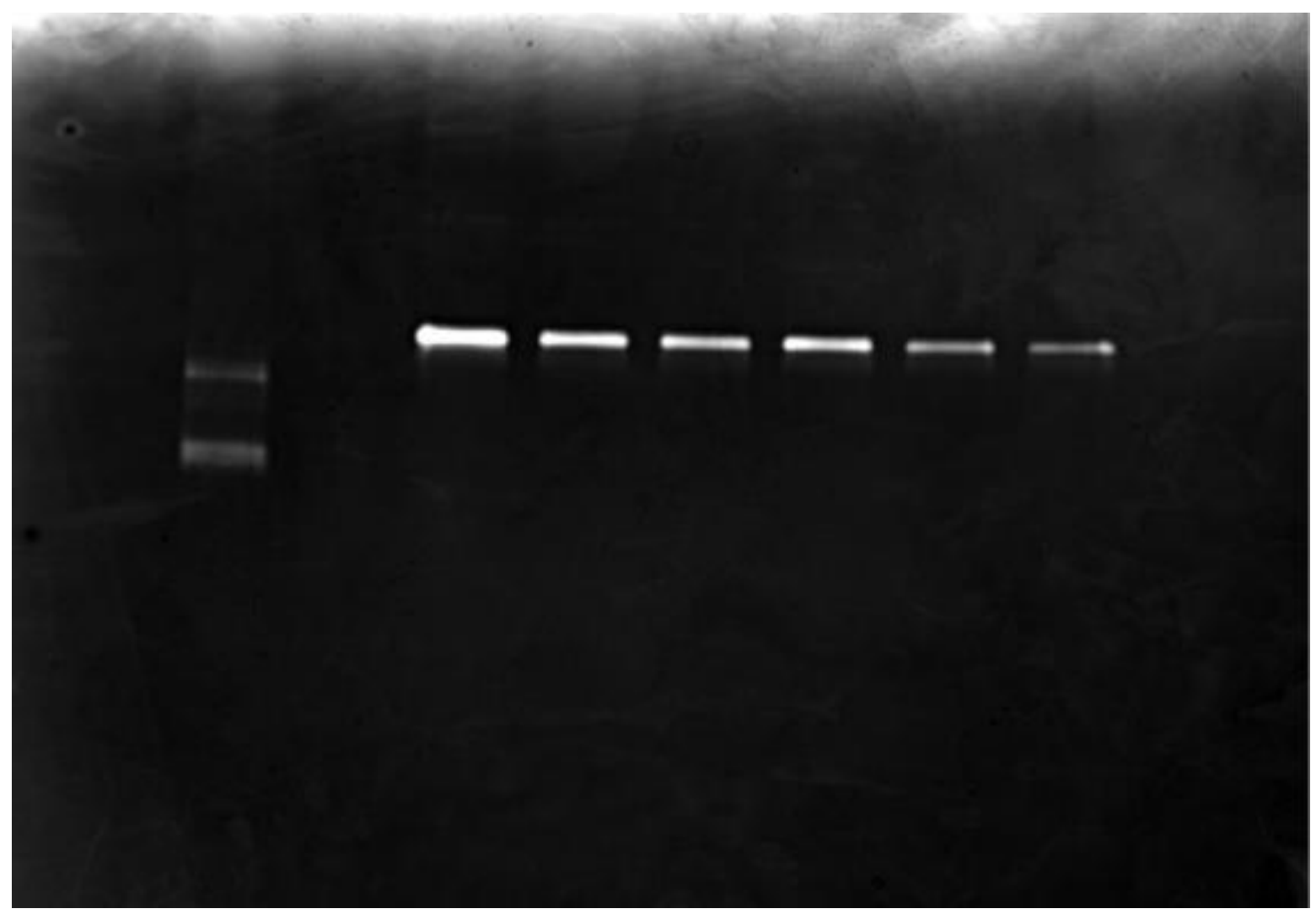

Supplementary Figure 3. Full Zymogram Gel of Cropped Image as Shown in Figure 1A. Using a 10well gel, MMP-9 Active, Human, Recombinant (Millipore Sigma; \#PF024-5UG) was used as a reference standard showed MMP-9 gelatinolytic activity at $67 \mathrm{kDa}$ (lane 1). DMSO at $0.1 \%$ was used as the vehicle control and constitutively showed MMP-9 gelatinolytic activity (lane 3). Upon MPA treatment, MMP-9 gelatinolytic activity was significantly decreased (lanes 4-7). GM6001 (lane 8) was used as a negative control for active MMP-9. 


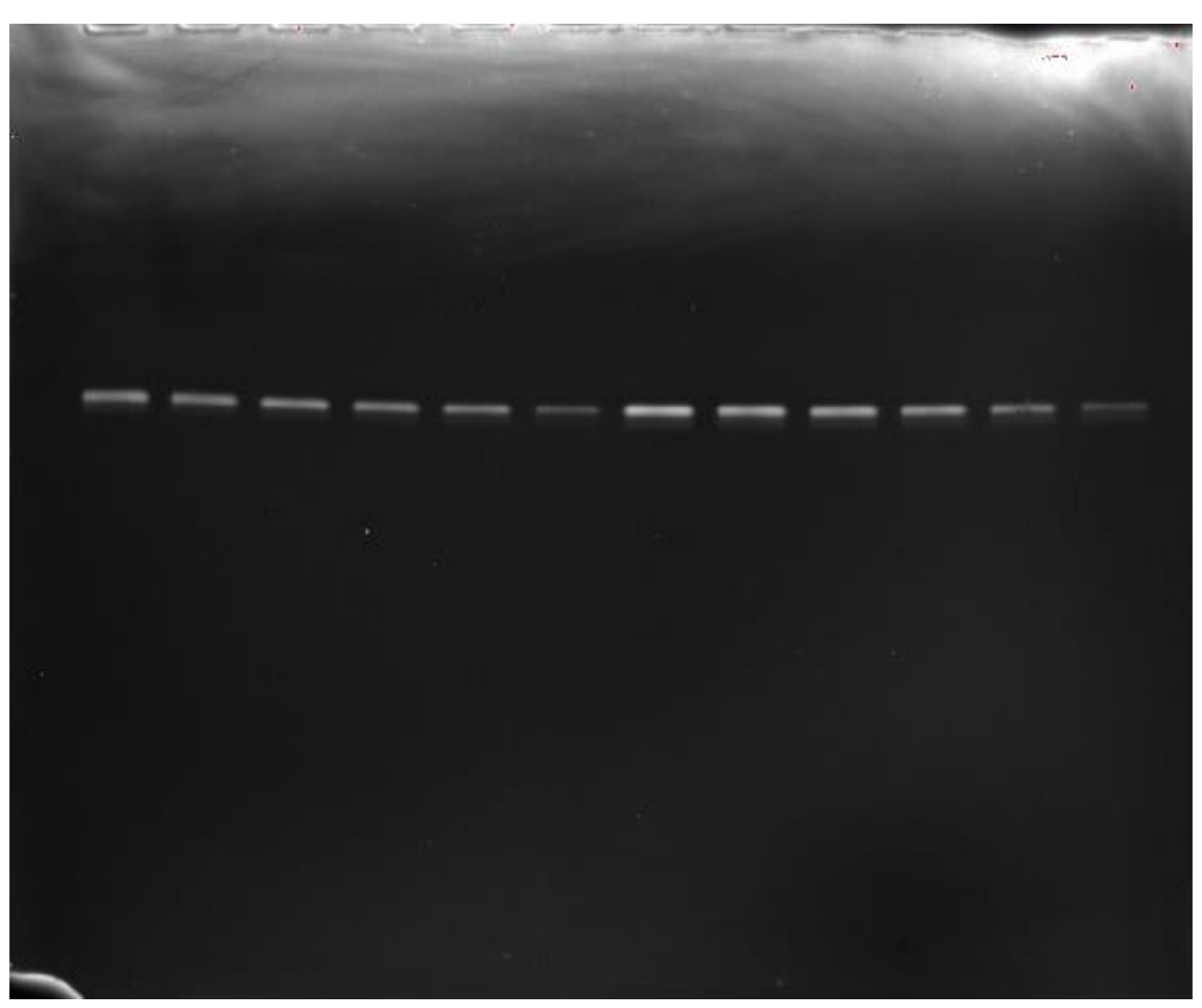

Supplementary Figure 4. Full Zymogram Gel of Cropped Image as Shown in Figure 1B. Using a 12well gel, DMSO at $0.1 \%$ was used as the vehicle control and constitutively showed MMP-9 gelatinolytic activity (lanes 1 and 7). Upon MPA treatment, MMP-9 gelatinolytic activity was significantly decreased (lanes 2-5 and 8-11). GM6001 (lanes 6 and 12) was used as a negative control for active MMP-9. 


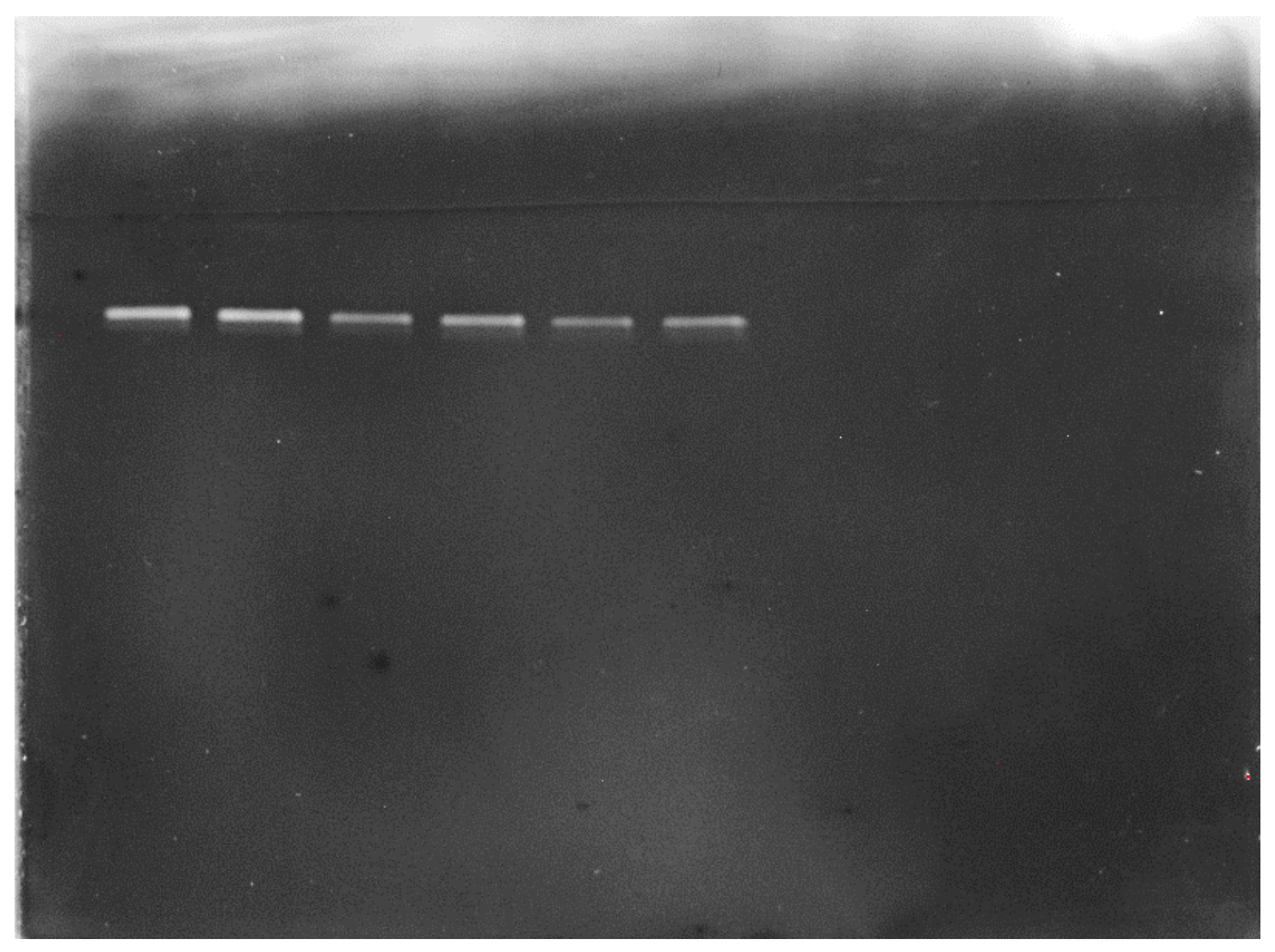

Supplementary Figure 5. Full Zymogram Gel of Cropped Image as Shown in Figure 4. Using a 10-well gel, DMSO at $0.1 \%$ was used as the vehicle control and constitutively showed MMP-9 gelatinolytic activity (lane 1). C6 cells were pretreated with mifepristone, RU486 (2 $\mu \mathrm{M})$, for $30 \mathrm{~min}$, followed by MPA treatment for another 48-h. Treatment with RU486, alone, did not alter MMP-9 enzymatic activity (lane 2). Upon MPA treatment, MMP-9 gelatinolytic activity was significantly decreased (100 $\mathrm{nM}$, lane 3; $10 \mu \mathrm{M}$, lane 5). Pretreatment with RU486 (2 $\mu \mathrm{M})$ antagonized MPA's effect on enzymatic activity (100 nM, lane 4; $10 \mu \mathrm{M}$, lane 6). 


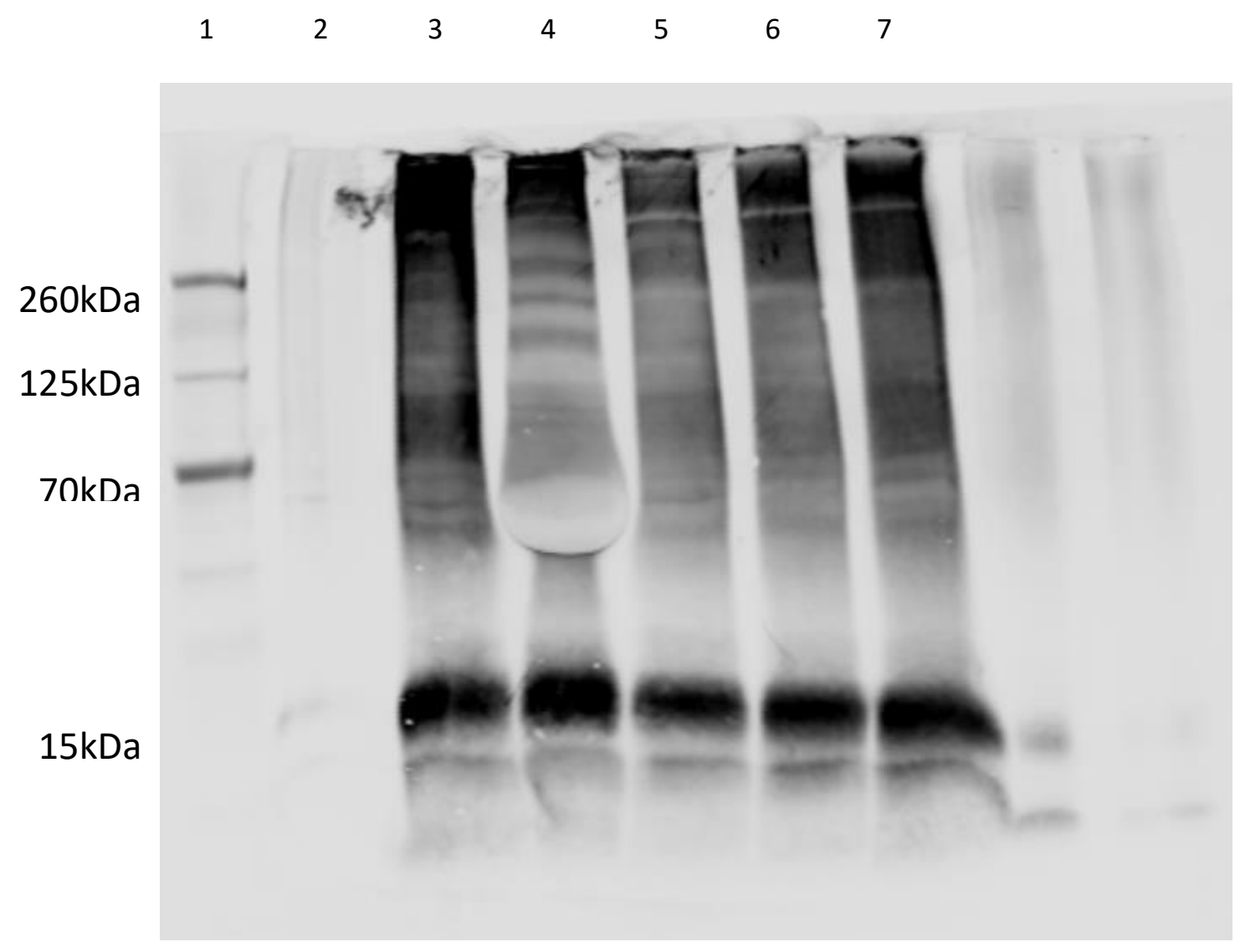

Supplementary Figure 6. Full Western Blot Gel of Cropped Image as Shown in Figure 5A. Li-Cor PreStained Protein Ladder (ChameleonTM Duo; \#928-60000) (lane 1). Freshly prepared synthetic human AB1-42 (23 $\mu \mathrm{M}$ ) was added to serum-free media (SFM) (lane 3), 10\% fetal bovine serum (FBS) media (lane 4), SFM that had been conditioned by incubation with C6 glial cells (CM) (lane 5), or to 100 nM MPA-treated CM (lane 6) or 10 M MPA-treated CM (lane 7). 


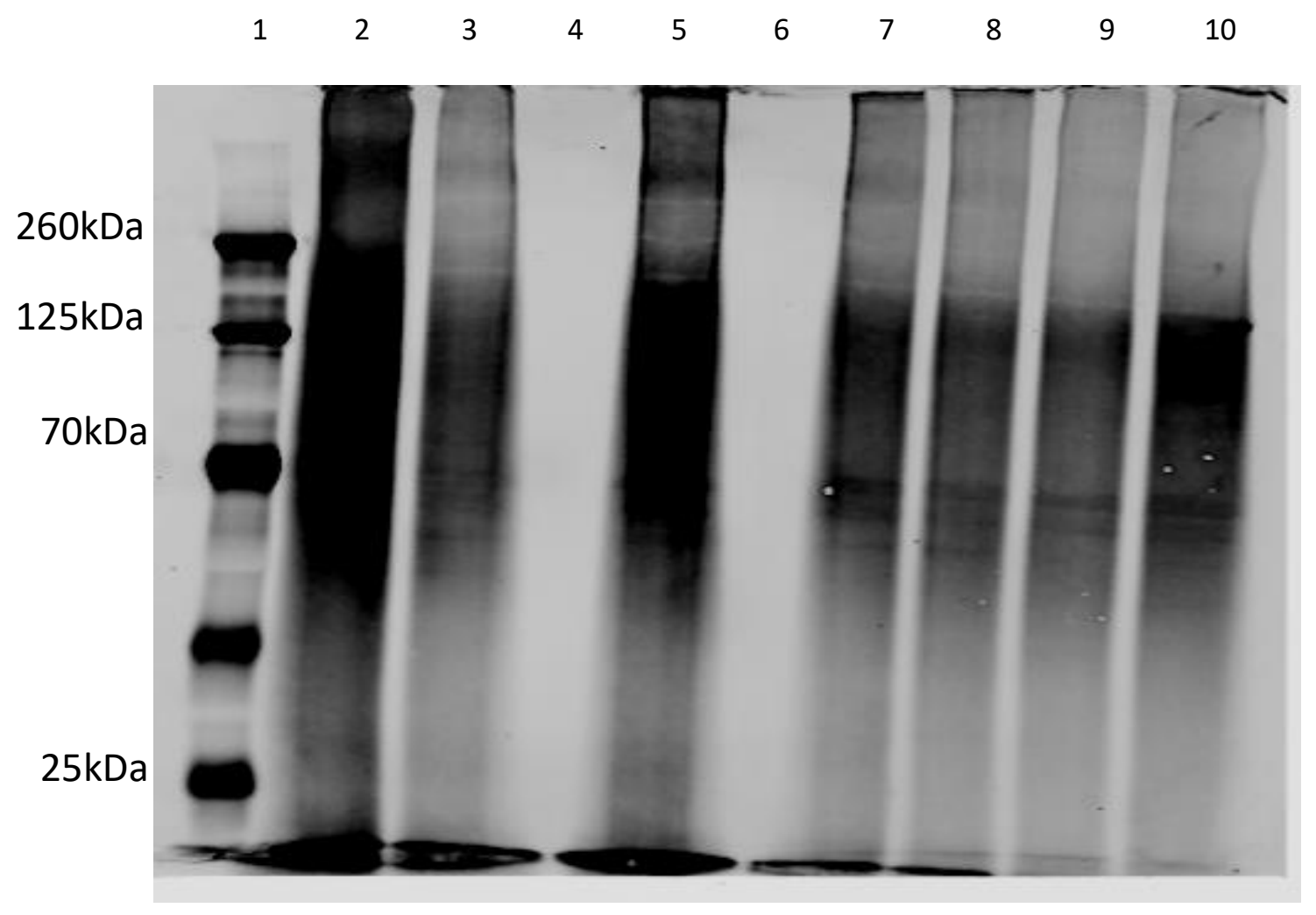

Supplementary Figure 7. Full Western Blot Gel of Cropped Image as Shown in Supplementary Figure 2. Li-Cor Pre-Stained Protein Ladder (ChameleonTM Duo; \#928-60000) (lane 1). Freshly prepared synthetic human Aß1-42 (23 $\mu \mathrm{M})$ was added to serum-free media (SFM) (lane 2), SFM that had been conditioned by incubation with $\mathrm{C} 6$ glial cells (CM) (lane 3), CM that had been treated with $1 \mu \mathrm{M}$ GM6001 (lane 5), or to CM that had been treated with MPA (10 nM, $100 \mathrm{nM}, 1 \mu \mathrm{M}$, and $10 \mu \mathrm{M})$ for 48-h (lanes 7-10). As noted in our Discussion, our synthetic $A \beta$ preparation exhibited variability in oligomer generation. The variability in aggregates stems from using HPLC grade water as our solvent, instead of alcohols, such as hexafluoroisopropanol (HFIP), which removes preexisting aggregates and beta sheet secondary structures from $A \beta 1-42$, yielding the peptide in one specific form of $A \beta 1-42$ species. The solvent used to dissolve the lyophilized peptides determines the initial conformation of amyloid beta and also the aggregation kinetics (Wei and Shea, 2006). Additionally, while performing replicates of our studies, our amyloid preparation was stored at -80 . Amyloid peptides are shown to be sensitive to temperature and freezing even at low concentrations, where freezing the peptide allows for uncontrolled oligomerization, causing the variation in aggregates once it goes through a freeze/thaw cycle (Filippov et al. 2008). We observed changes in the preparation as an effect of time and temperature. During our experiments and replicates, we found that if the preparation was not freshly made with each replicate, our Western blot detection would then lack the sensitivity required to detect MPA-induced effects on degradation. The effects of CM and GM600, however, could still be detected, using a synthetic preparation that was not freshly made. 


\section{Chapter 3}

\section{The In Vivo Effects of Long-Term Administration of Medroxyprogesterone Acetate on Matrix Metalloproteinases in Cognitive Function}

Keyana N. Porter, Deborah Corbin, Saumyendra N. Sarkar, Dominic D. Quintana, and James W. Simpkins 


\subsection{Abstract}

Progestins, outside of their action on the endometrium, have multiple nonreproductive functions. Currently, the literature provides only a superficial understanding of the interplay between synthetic progestins and the central nervous system (CNS). While the central effects of progesterone are wellcharacterized, the effects of synthetic progestins on the brain are understudied. Recently, we have demonstrated that in vitro treatment of glial cells with medroxyprogesterone acetate (MPA), a widely used synthetic progestin, resulted in repression of matrix metalloproteinase-9 (MMP-9) expression and activity, as measured by their capabilities to degrade amyloid beta. Matrix metalloproteinases (MMPs) are expressed by neurons and glial cells, in the adult brain, and released in response to enhanced neuronal activity under physiological and pathological conditions. Moreover, synaptic connections rely heavily on the function of MMP-9. We reasoned that MPA's suppression of MMP-9 expression would also negatively impact cognitive and synaptic function, through the downregulation of MMP-9. The present investigation evaluated the long-term effects of MPA on MMP-2/-9 and cognitive function in intact, wild-type (WT) mice and the triple transgenic (3xTg-AD) mouse model of AD. We hypothesized that long-term administration of MPA accelerates the onset of cognitive dysfunctions in 3xTg-AD mice. Additionally, we hypothesized MPA would induce cognitive impairments in the WT mice, through negative impacts on synaptic function via action on MMP-9. Our results indicate that MPA treatment promotes cognitive dysfunction and increases cortical MMP-9, in vivo. These results raise the possibilities of yielding similar effects in women who exercise the chronic use of MPA to prevent pregnancy or in HT formulations. 


\subsection{Introduction}

Alzheimer's disease (AD) is the most prevalent form of dementia, characterized by progressive cognitive decline. The pathological hallmarks of $A D$ are extracellular plaques stemming from the accumulation of amyloid beta, neurofibrillary tangles as a result of aggregated hyperphosphorylated tau, neurodegeneration, and the loss of synapses. As the main constituent of amyloid plaques, $A \beta$ plays a key role in the pathogenesis of $A D$ (Selkoe et al., 2001). Accumulation of $A \beta$ occurs nearly a decade prior to the onset of clinical symptoms and originates from the proteolytic cleavage of amyloid precursor protein (APP), by beta- and gamma-secretases (Scheltens et al., 2016), which promotes the amyloidogenic cleavage of APP. This produces 40 or 42 amino acid fragments of $A \beta\left(A \beta_{1-40}\right.$ and $\left.A \beta_{1-42}\right)$ and subsequently, A $\beta$ peptide is released into the extracellular space (Chen, 2015; Xu et al., 2016). Extracellular $A \beta$ assumes several conformational states ranging from monomers to soluble oligomers and fibrils, which rapidly form aggregates and ultimately amyloid plaques which are characteristic of the disease (Pryor et al., 2012). In a series of experimental models of $A D$ and in $A D$ patients, there is a growing body of evidence that neuronal dysfunction stems from soluble oligomeric $A \beta$ (Mucke et al., 2000; Gong et al., 2003; Cheng et al, 2007). APP is also recognized by alpha-secretase ( $\alpha$-secretase), which cleaves the precursor protein, promoting the non-amyloidogenic cleavage of APP and producing the Nterminal fragment soluble APP $\alpha$ (sAPP $\alpha$ ). sAPP $\alpha$ has been reported to promote neuroprotection and cognitive resilience (Ghiso and Frangione, 2002). Decreased expression of SAPPa in the cerebrospinal fluid (CSF) of familial (FAD) and sporadic AD (SAD) patients (Lannfelt et al., 1995; Almkvist et al., 1997; Colciaghi et al., 2002; Fellgiebel et al., 2009), due 
to reduced $\alpha$-secretase activity and increased $\beta$-secretase activity (Tyler et al., 2002), is highly correlated to memory impairments in aged rodents and AD patients (Almkvist et al., 1997; Anderson et al., 1999; Fellgiebel et al., 2009).

MMPs are a family of zinc-dependent enzymes responsible for the degradation and remodeling of extracellular matrices (ECM). In AD, MMP-2 and MMP-9 facilitate the clearance and degradation of $A \beta$, influence the aggregation kinetics of tau protein, and additionally regulate synaptic connections (Deb and Gottschall, 1996; Deb et al., 2003; Talamagas et al., 2007; Mizoguchi et al., 2009). In contrast to other A $\beta$-degrading proteases, MMP-9 is known to degrade $A \beta$ fibrils in vitro, $A \beta$ plaques in situ, and $A \beta$ in vivo (Backstrom et al., 1996; Yan et al., 2006; Yin et al., 2006; Hernandez-Guillamon et al., 2015). Overexpression of MMP-9 in a transgenic mouse model of AD (5xFAD) resulted in reduced levels of oligomeric $A \beta$ and cognitive resilience, in specifically female animals when compared to their male counterparts. Additionally, MMP-9 overexpression caused increased levels of SAPP $\alpha$, via enhanced cleavage of APP at $\alpha$-secretase cleaving sites. In theory, modulation of MMP-9 levels potentially impacts the progression of AD pathogenesis. Conversely, MMP-9 has been shown to be increased in AD brains (Backstrom et al., 1996). Moreover, there are reports that treatment with a non-specific MMP inhibitor, GM6001, improves A $\beta$-induce cognitive impairment and neurotoxicity, in vivo. The authors hypothesize MMP-9 likely performs nonselective cleavage of the ECM and neural membranes, leading to neuronal dysfunction and cognitive impairment and suggesting a causal role of MMP-9 in A $\beta$-induced cognitive deficits and neurotoxicity (Mizoguchi et al., 
2009). Despite the discrepancies within the literature, taken together, modulating the levels of MMP-9 impacts AD pathogenesis.

Medroxyprogesterone Acetate (MPA) is globally used as the progestin component of hormonal therapy used by menopausal women, Prempro $^{\circledR}$ and Premphase ${ }^{\circledR}$, as well as a hormonal contraceptive, Depo Provera ${ }^{\circledR}$. There is accumulating evidence that MPA increases the risk of dementia for menopausal women (Shumaker et al., 2003, 2004), induce cognitive impairment in ovariectomized animal models (Braden et al., 2010, 2011, 2017), and exert amnestic effects in case studies of premenopausal women (Gabriel and Fahim et al., 2005). MPA's cognitive-impairing effects may be mediated via its action on modulating the levels of MMPs. In vitro evidence suggests that estrogen-related compounds induce MMP-2 and MMP-9 expression, promoting the degradation of $A \beta$ and produce neuroprotective effects (Merlo et al., 2012). We have previously reported that hormonal modulation of MMP-9, utilizing MPA, decreases the production and proteolytic activity of MMP-9, in turn, impairing the A $\beta$-degrading activity of MMP-9 (Porter et al., 2020). Alarmingly, the long-term consequences of using MPA during a woman's reproductive prime is understudied, as a majority of the previous investigations utilized menopausal animal models and women during the stages of menopause. In this study, we used a transgenic mouse model of AD to investigate the effects of chronic MPA treatment, in intact animals, on cognitive function. Our central hypothesis is that MPA-treated animals, both the WT and AD groups, will show cognitive impairments. Here, we demonstrate MPA promotes differential effects on memory consolidation. MPA treatment induced a modest memory impairment in WT animals, while yielding a beneficial effect in 3xTg-AD animals. 


\subsection{Materials and Methods Animals and drug treatments}

Triple $\mathrm{APP}_{\text {swe, }} \mathrm{PS}_{\mathrm{M} 146 \mathrm{~V}}$ and Taup301L transgenic mice (3xTg-AD) and wild-type (WT;

B61129SF2/J) mice were obtained from Jackson Laboratory (Bar Harbor, Maine, USA). The mice were maintained at constant temperature with an alternating 12 hours light-dark cycle. Food and water were available ad libitum. Twenty mice were assessed in this study; female 3xTg-AD $(n=10)$ and WT $(n=10)$. Mice (5 animals/group) were randomly assigned and injected subcutaneously (s.c.) with either saline or $4 \mathrm{mg}$ depot medroxyprogesterone acetate (MPA; Pfizer, New York, NY) once every 4 weeks, from the age of 3-months to 6months. This dose was selected to approximate serum MPA levels achieved by DepoProvera injection for prevention of undesired pregnancy. The $4 \mathrm{mg}$ dose also produces mean serum concentration of $21.37 \mathrm{ng} / \mathrm{ml}$, respectively (the level that approximates the peak serum concentrations of MPA in women using Depo-Provera for prevention of undesired pregnancy) (Miguel et al., 2012). Control mice received the same volume of vehicle. To confirm the effectiveness of MPA injections on ovulation, vaginal smears were taken for the seven days post-injection 1 . Smears were classified as proestrus, estrous, metestrus, or diestrous per prior protocol (Goldman et al., 2007; Acosta et al., 2009). Animals treated with MPA were confirmed to be in a constant diestrous phase, while vehicle-treated animals showed a normal cyclicity. All animal experiments were performed in accordance with the National Institutes of Health guide for the care and use of laboratory animals. The animal studies were approved by the Institutional Animal Care and Use Committee of West Virginia University. 


\section{Morris Water Maze}

After the last injection, mice were tested in the Morris water-maze (MWM) task. The apparatus and the protocol used a circular pool (140 cm in diameter) situated in a room with discrete extramaze cues and filled with water $\left(23 \pm 1^{\circ} \mathrm{C}\right)$, that was rendered opaque by the addition of white paint. A clear movable platform (10 cm in diameter) was submerged 1 $\mathrm{cm}$ below the water surface. The protocol we followed consisted of a 1-day visible phase (four 60-second trials per day in the presence of a movable visible platform), a 4-day acquisition phase (four 60-second trials per day in the presence of a hidden platform, located in a fixed position (NE quadrant) relative to visible extramaze cues) and a 2-day reversal phase (opposite to the target quadrant i.e. the quadrant where the platform was located during acquisition). Each daily session consisted of four trials and four different starting positions were used in a random order. Each trial had a maximum duration of $60 \mathrm{~s}$ and mice who failed to locate the platform within $60 \mathrm{~s}$ were gently placed on the platform. At the end of each trial, mice remained on the platform for $15 \mathrm{~s}$. The behavior of animals during the water-maze was analyzed by the behavioral analysis system AnyMaze (Stoelting, Wood. Dale, IL, USA). In order to assess learning in the visible and the acquisition phase of the task, two parameters were analyzed; latency (time in seconds to reach the platform) and swim distance (in meters). The values obtained were averaged per mouse within each daily session. To ensure that behavior in the water-maze did not simply reflect changes in activity, swim speed was also scored; these values were also averaged per mouse within each of the phases of the task (visible, acquisition and reversal). 


\section{Enzyme-linked immunosorbent assay (ELISA)}

Hemispheric tissue samples were weighed and frozen immediately in liquid nitrogen and stored at $-80^{\circ} \mathrm{C}$. Using a ratio of $\sim 1 \mathrm{~g}$ of tissue to $20 \mathrm{~mL}$ reagent, total proteins were extracted using T-PER tissue protein extraction reagent (Thermo Scientific) added with Halt Protease Inhibitor Cocktail (Thermo Scientific) and 1 mM EDTA (Invitrogen). Supernatant was collected after centrifugation at $10,000 \mathrm{~g}$ for $15 \mathrm{~min}$ at $4^{\circ} \mathrm{C}$, the total protein concentrations were measured using a BCA protein assay kit (Thermo Scientific), and samples were normalized to $65 \mu \mathrm{g} / \mathrm{mL}$ of total protein. Levels of MMP-2 and MMP-9 in mouse hippocampal and cortical homogenates were analyzed using commercially available high-sensitivity ELISA kits (Quantikine, R\&D Systems), according to the manufacturer's instructions. The optical density in each well was read at $450 \mathrm{~nm}$ (with $540 \mathrm{~nm}$ correction) using a microplate reader.

Blood samples were drawn via cardiac puncture into heparinized before perfusion. Plasma was isolated from blood in heparinized tubes, on ice. Samples were then centrifuged for 20 minutes at $2000 \times \mathrm{g}$ within 30 minutes of collection. An additional centrifugation step of the plasma at $10,000 \times \mathrm{g}$ for 10 minutes at $2-8^{\circ} \mathrm{C}$ is recommended for complete platelet removal. Plasma samples were aliquoted and stored at at $-80^{\circ} \mathrm{C}$ until analysis. All measurements were done in duplicate. Similar standard curves for each of the proteins were required in each plot. The immunoassays were carried out following the manufacturer's instructions. All assays were done in duplicate. 


\subsection{Results}

\section{In Vivo Assessment of Medroxyprogesterone Acetate-Induced Effects on Memory and}

\section{Learning}

To test the effects of MPA on memory and spatial learning impairments, we performed the Morris Water Maze test (Figure 1). The acquisition trials (hidden platform: HP) of the MWM task is used to assess learning capacity, which analyzes the escape latency (time required to reach the platform) (Figure 2 and 3) and swimming distance to the platform (Figure 4). Our latency data reveals a significant MPA-induced learning deficit in the WT animals on Day 1 of reversal training (Figure $2 \mathrm{~A}$ ), and this is controlled by similar rates of swim speed (Figure 2B). Additionally, on the final day of acquisition training, MPA enhanced learning in AD animals (Figure $3 \mathrm{~A}$ ) and this is controlled for with no significant differences in swim speed (Figure 3B). On the specific days where we observed significant differences in swim speed (Figure $2 \mathrm{~B}$ and $3 \mathrm{~B}$ ), we were unable to interpret escape latency data without existing confounding factors and solely relied on the analysis of swim distance across all trials. Swim distance was averaged across all 5 trials, for Day 1 and Day 4, the initial and final days of acquisition training. The WT animals treated and untreated, both successfully improved in performance over the course of training, as indicated by the significant reductions in their swim distances (Figure 4A). However, treatment with MPA revealed a modest enhancement in spatial memory/learning, in $A D$ animals (Figure 4B). We compared performances on trial 1 only, of the first and fourth day (final) of acquisition training (Figure 4C, D), to reflect memory retention and memory consolidation. In the WT animals, the swim distance of vehicle-treated animals significantly decreased from Trial 1 of Day 1 to Trial 1 of Day 4 
(Figure 4C). However, this effect was not seen in the WT- MPA animals. Although

statistically insignificant, MPA-treated WT animals had increased swim distance on Trial 1 of Day 4 of training, relative to vehicle-treated animals. However, in AD animals, MPA-treated animals show improved memory consolidation, as their swim distance in significantly reduced, from Trial 1 of Day 1 to Trial 1 of Day 4 (Figure 4D). Trial 5 of Day 1 and Day 4 is indicative of short-term or working memory, and all our animals show no effect of treatment in those trials (data not shown).

There were no statistically significant differences in swim distance between groups in the reversal training (Figure 4E, F). Noticeably, average swim distance, on Day 1 of Reversal training, shows WT mice treated with MPA appear to not entirely abandon their initial learning, though the differences in swim speed are not statistically significant (Figure $2 \mathrm{~A}$ and 4E). There were no major differences in swim distance observed in the AD animals (Figure 4F). By Day 2 of Reversal training, all four animal groups had relatively low swim distances, with no significant effects of treatment (data not shown).

The entries into the former location of the platform (NE) were quantified in order to assess cognitive flexibility. The untreated WT animals show a significant reduction in the number of entries they made into the NE quadrant, while the target quadrant was now the South, but the statistical significance of this effect is not observed in the MPA-treated WT animals (Figure 5A). Additionally, although statistically insignificant, MPA-treated WT animals had higher number of entries into the previous platform zone (NE quadrant), Day 2, Trial 5 (Figure 5C). There was no effect of treatment on the number of entries, in the AD animals 
(Figure 5B, D). Collectively, these data imply MPA positively impacts memory consolidation in $A D$ animals, while only potentially impairing memory consolidation in the acquisition training and cognitive flexibility during the reversal training, in WT animals.

\section{The Effect of MPA on MMP-2/-9 Expression}

The levels of MMPs are modulated by a variety of stimuli, including hormones that are utilized as either contraception or menopausal hormone therapy (HT), both natural and synthetic. To study the effect of Medroxyprogesterone Acetate (MPA) on matrix metalloproteinase-2 and -9 (MMP-2/-9) expression, we administered MPA subcutaneously to WT and 3xTg-AD mice, once a month, for 3 consecutive months. MMP-2 and MMP-9 have been strongly associated with learning and memory in past studies. To measure hippocampal and cortical MMP-2/-9 protein levels, brain homogenates were analyzed by ELISA for MMP-2/-9 (pro-MMP-2/-9, TIMP bound MMP-2/-9, and latent MMP-2/-9; Figures 6 and 7). Our analysis showed that MPA significantly reduced protein expression of MMP-2 in the frontal cortex (Figure 6C) and the hippocampus (Figure 6D) of AD animals but show no effect of treatment on MMP-2 in WT animals (Figure 6A, B). MPA treatment significantly increased protein expression of MMP-9 in the frontal cortices of both WT and AD animals, with a more robust effect in WT animals. A 2-fold increase in WT MPA animals was shown, relative to control (Figure 7A), while MMP-9 expression in AD animals was also increased, to a lesser extent (Figure 7C). There were no effects of treatment observed in the hippocampal samples of either genotype of animals (Figure 7B, D). 


\subsection{Discussion}

The present study investigated the effects of MPA administration on WT and 3xTg-AD mice. Several reports have demonstrated that MPA can alter MMP-9 levels, where levels are increased in macrophages and, conversely, decreased in several other cell lines (Di Nezza et al., 2003; Hwang-Levine et al., 2011; Allen et al., 2019). We have previously shown that 48 and 72-hour exposure of C6 glial cells to MPA decreased MMP-9 protein expression and enzymatic activity (Porter et al., 2020). In our previous study, we used a relatively acute treatment paradigm, while in our current study we investigated the chronic effects of MPA treatment in vivo. Furthermore, most of the previous in vivo studies have used ovariectomized female animals to observe the effect of MPA on the brain, with little to no investigation of the effects of MPA on brain MMP-9. Additionally, due to the lack of focus in a CNS-related system, previous work failed to investigate the potential for MPA to influence cognitive function and AD-related pathology via the hormonal modulation of MMP-9. Herein, we provide data showing the effects on brain MMP profiles and cognitive function in intact female mice.

To our knowledge, this is the first in vivo investigation of the effect of chronic MPA administration on brain MMP-2/-9's expression, in vivo. Our results show that MMP-9 is expressed in the brains of WT and AD animals in the hippocampus and cortex of vehicletreated animals and is increased by exposure to MPA at the protein level. Interestingly, the observed effects go against our original hypothesis, as we confirmed stimulatory effects of MPA on the expression of MMP-9, whereas in the glial cell line, we observed inhibitory effects of MPA, as shown by the suppression of transcription of MMP-9 at the mRNA level. 
Conversely, we found MPA significantly reduced hippocampal and cortical MMP-2 protein expression, in the $A D$ animals, however the biological significance of our observations is minimal despite their statistical significance.

In the cytoplasm, the glucocorticoid receptor (GR) is considered inactive, until a ligand for the receptor binds and activates the receptor. In turn, this typically promotes an anti-inflammatory response through the induction of anti-inflammatory gene expression or transrepression of proinflammatory genes. Recent work from our group revealed MPA's effects on MMP-9 expression are modulated in a GR-dependent manner, where the pretreatment of C6 glial cells with a GR antagonist (RU486), prior to MPA exposure, attenuates MPA's effects on MMP-9 expression (Porter et al., 2020). Moreover, glucocorticoids have been shown to alter MMP-9 expression (Rosenberg et al., 1996; Park et al., 1999; Eberhardt et al., 2002; de Paiva et al., 2006), as well as MMP-3 (Richardson and Dodge, 2003; Koyama et al., 2017), which is the enzyme responsible for the conversion of MMP-9 from an inactive to an active state. The literature extensively supports the notion that MPA binds to the GR with a high affinity, acting as a partial to a full agonist for the GR (Koubovec et al., 2004, 2005; Su et al., 2012a, b; Louw-du Toit et al., 2014). Alternatively, Zhao et al. indicates MPA's agonistic behavior is highly dependent on the expression levels of the GR. Decreasing the expression levels of the GR converts MPA from an agonist to an antagonist of GR-mediated transrepression (Zhao et al., 2003). Therefore, it is plausible that the discrepancy between the effects seen in our in vitro study and our in vivo study are attributed to alterations in the expression of the GR, as an effect of our chronic MPA 
treatment, in both the WT and AD animals. Future studies should aim to further delineate the precise mechanisms by which our observations in the present study occur.

To our knowledge, this is the first investigation of the effects of MPA treatment on cognitive function, and AD-related pathology, in intact female animals. Our objective was to relate progestin-induced elevations of active MMP-9 levels with cognitive impairments. We were interested in observing the effects of monthly subcutaneous injections and, using the Morris Water Maze task, we were able to confirm the differential effects on memory consolidation on WT and 3xTg-AD animals.

Our most significant behavior data suggests MPA-treated AD animals were more capable of retaining memory of the platform's location overnight. We sought out to investigate the effect of chronic MPA treatment on cognitive function in the 3xTg-AD mouse model and found a significant effect on memory consolidation and cortical MMP-9 expression. In the transgenic mouse model of AD which has five familial AD-related mutations (5xFAD), overexpression of MMP-9 reduced oligomeric $A \beta$, increased synaptic markers, and prevented cognitive deficits (Fragkouli et al., 2014; Kaminari et al., 2017). Our finding that MMP-9 expression is increased in AD animals, after chronic treatment of MPA, is in line with findings in the literature, where increased MMP-9 expression yields beneficial effects in $A D$ animal models. Although we did not statistically compare the two genotypes used in this study, we noticed the performances between the two vehicle treated groups were not vastly different. The literature states 6 -months of age in this specific animal model should have sufficed to induce obvious impairments in $A D$ animals performing the MWM 
task (Oddo et al., 2003). It is possible that our AD animals may have been selected at a time before an apparent learning and/or memory deficit.

Within our vehicle groups, we assessed the effect of MPA treatment on performance between the first and final day of acquisition training. When swim distance is averaged across all 5 trials, for Day 1 and Day 4, both the vehicle and MPA-treated groups improved over the course of training. However, if we average the performances for Trial 1 of Day 1 and Day 4, the WT vehicle animals show they are progressively retaining information, and this significant change is not observed in the MPA-treated animals. This suggests MPA treatment impairs memory retention in WT animals, but modestly enhances memory consolidation and working memory in AD animals.

Cognitive flexibility is consistently associated with damage to the prefrontal cortex (PFC) (Ragozzino et al., 1999; Birrell and Brown, 2000; Block et al., 2007; Bissonette et al., 2008). Additionally, the frontal cortex is involved in working memory, cognitive flexibility, attention and other cognitive and executive functions that are affected both in patients and animal models with AD (Baddeley et al., 1991; Brugger et al., 1996; Perry and Hodges, 1999). Cognitive flexibility is the ability to update a strategy or adjust a previously learned behavior in response to alterations in a present task (Bissonette et al., 2013). In the MWM, evidence of loss of cognitive flexibility is shown when treated animals failed to adapt their behavior to learn the new location of the platform, after switching it from the target quadrant (NE) to the opposite quadrant. Reversal learning reveals whether animals can extinguish their initial learning of the platform's location and possess cognitive flexibility to 
acquire the new location of the platform. After multiple trials on Day 1 of reversal training, WT mice treated with MPA appear not to entirely abandon their initial learning, though this difference is also not statistically significant. There were no major differences observed in the AD animals. By Day 2 of reversal training, all four animal groups were shown to be capable of uncovering the new location, as shown by lower values of swim distance. Again, although statistically insignificant because of variability within the treatment group, on the last trial of the last day of Reversal training, a portion of the MPA-treated animals continued to enter the previously platformed zone (NE). At this point in training, animals should have grasped the concept that there is no longer any reason to enter the NE quadrant. Figure 4 shows all the other treatment groups have relatively lower number of entries, with less variability within the group.

Our finding that chronic treatment of MPA elevates MMP-9 expression, in WT animals, could ultimately negatively impact the perineuronal net (PNN), as MMP-9 is considered a degrading enzyme for proteins within the PNN. Increased disruption of the PNN in the PFC leads to deficits in cortical GABAergic signaling (Slaker et al., 2015; Sorg et al., 2016; Bozzelli et al., 2018; Paylor et al., 2018; Wen et al., 2018; Holter et al., 2019). Parvalbumin (PV) GABAergic neurons in the PFC are also highly associated with cognitive flexibility (Murray et al., 2015). Thus, decreased GABA levels could play a role in the modest differences observed in the WT animals' MWM performances, correlating their behaviors to the 2-fold increased MMP-9 expression in their cortices. Herein, we encourage additional investigations related to MPA's effects on the PNN. It is conceivable that the chronic use of MPA will progressively increase the proteolytic degradation of the PNN by MMP-9, in vivo, 
which could potentially promote cognitive dysfunctions in memory consolidation and cognitive flexibility.

Our findings imply MPA positively impacts memory consolidation in AD animals. Additionally, because of our lack of statistical power and low sample size, our data are only able to suggest MPA potentially impairs memory consolidation in the acquisition training and cognitive flexibility during the reversal training, in WT animals. The major limitation of our study is the sample size. Although we had sufficient statistical power for our biochemical analyses, unfortunately we did not for our behavior studies. The inclusion of a behavior task more sensitive or tailored towards assessing cognitive flexibility and/or deficits within the frontal cortex is needed.

Although we were able to measure protein, we were unable to make the correlation of protein with enzymatic activity, due to our inability to detect it via zymography. Future studies could assess MMP-9 activity in histological sections via in situ zymography, where frozen sections are coated with fluorescently labeled matrix proteins, incubated, and observed for black patches in the fluorescent background, indicating proteolysis of the matrix protein (George and Johnson, 2010). Alternatively, frozen sections could also be incubated with dye-quenched (DQ) gelatin, which would become fluorescent upon proteolytic cleavage by MMP-9. In this method, MMP-9 activity would be denoted as bright fluorescent spots (Gawlak et al., 2009). We suggest future studies be performed, with a higher sample size, a more in-depth analysis of how MPA alters MMP-9 protein levels and what the consequences are. 
There is currently an unmet need for extensive in vivo investigation for the potential cognitive and pathological outcomes of MPA, in animals that are not ovariectomized. The majority of the literature focuses on the effects of estrogen, estradiol, testosterone, and progesterone on the brain, and particularly amyloid-beta production and clearance (Vest and Pike, 2013; Li and Singh, 2014; Giatti et al., 2016; Uchoa et al., 2016). There is still a paucity of published research addressing the potential effects of progestins, more specifically MPA, on the brain. While limited research with MPA suggests a negative impact on the brain, these studies need to be extended to model systems relevant to women in their reproductive prime, as a majority of efforts have been in menopausal animal models and menopausal woman (Shumaker et al., 2003, 2004; Braden et al., 2010, 2011, 2017; Lowry et al., 2010; Akinloye Olanrewaju et al., 2013). In summary, our work demonstrates the necessity for a further delineation of the consequences of chronic MPA administration's effects on brain MMP-9 and cognitive function, in WT and AD mice. Herein, we encourage additional, elaborate investigations related to MPA's effects on the brain. Future efforts should remain extended to model systems relevant to AD-related pathology, with a stronger sample size, and investigate the role of the PNN. 


\subsection{Figures}

\begin{tabular}{|c|c|c|c|c|c|c|c|}
\hline Phase & $\begin{array}{c}\text { Visible } \\
\text { Platform }\end{array}$ & \multicolumn{3}{|c|}{ Acquisition- Hidden Platform } & \multicolumn{2}{c|}{ Reversal Training } \\
\hline Day & 1 & 2 & 3 & 4 & 5 & 6 & 7 \\
\hline
\end{tabular}

A

Acquisition

B

\section{Reversal}
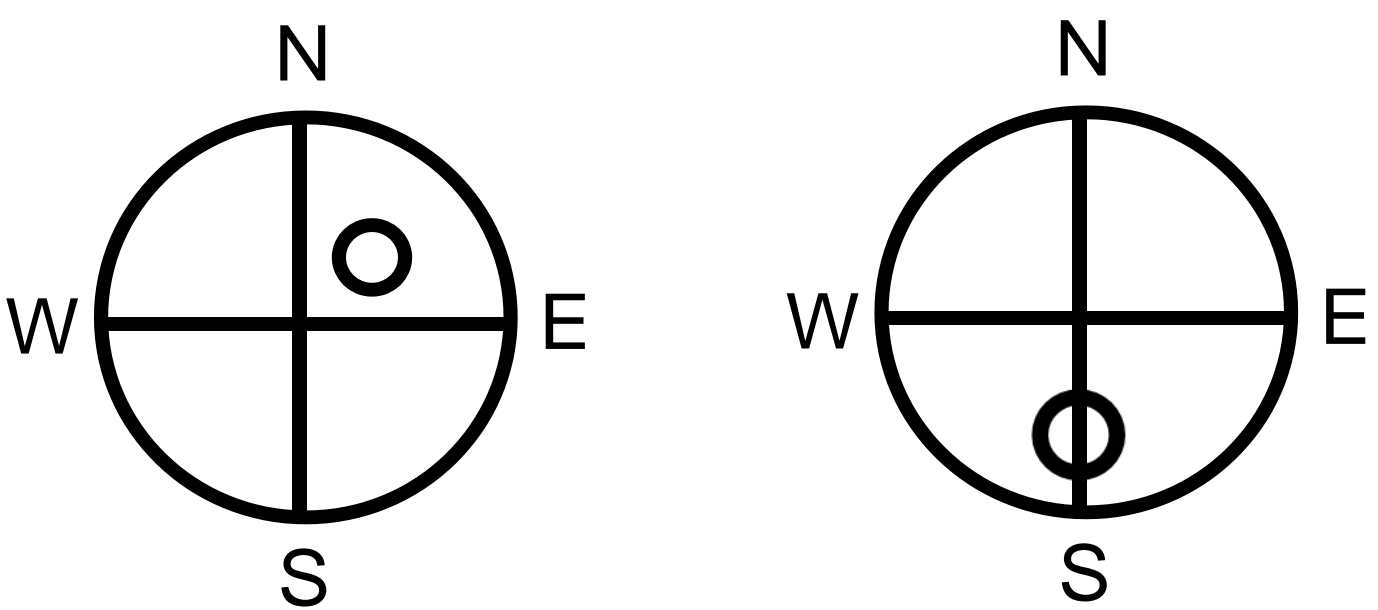

Figure 1. Outline of Morris Water Maze (MWM) task. (A) Acquisition training platform location: NE (B) Reversal training platform location: $\mathrm{S}$ 
A

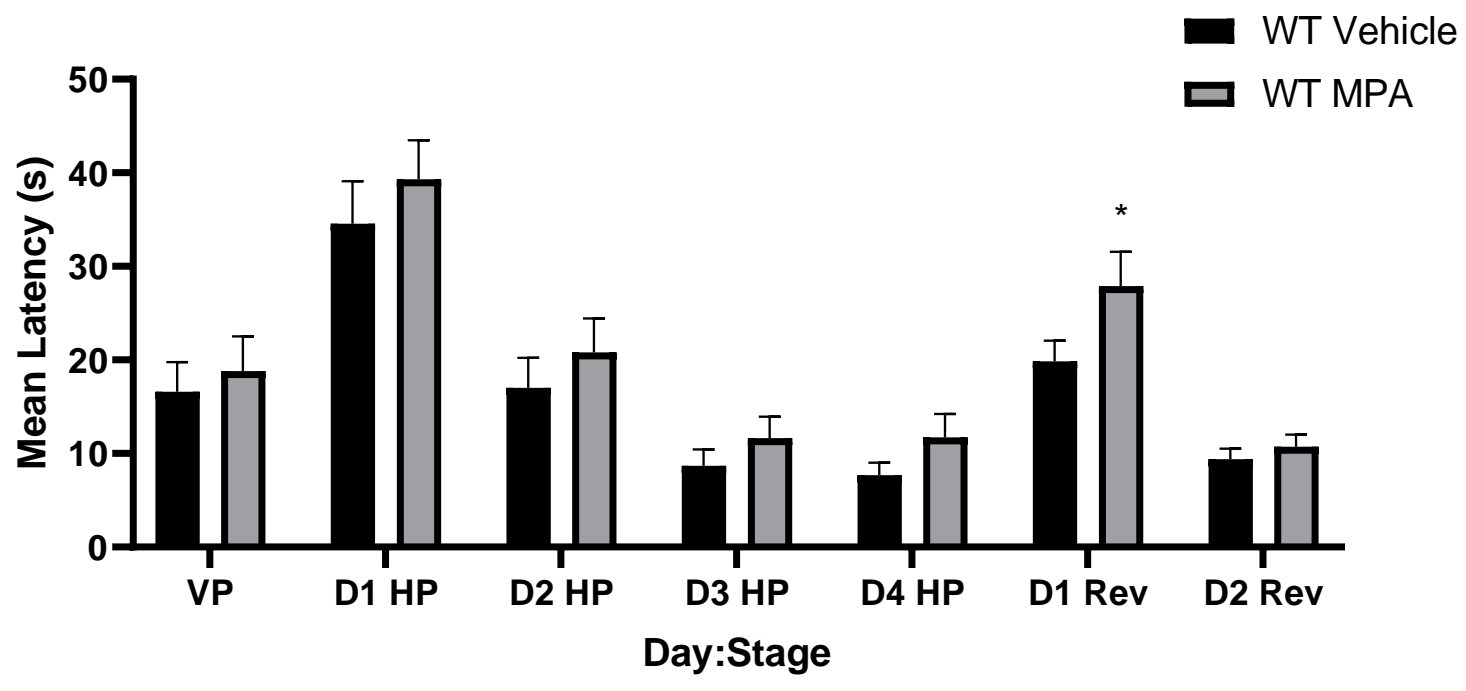

B

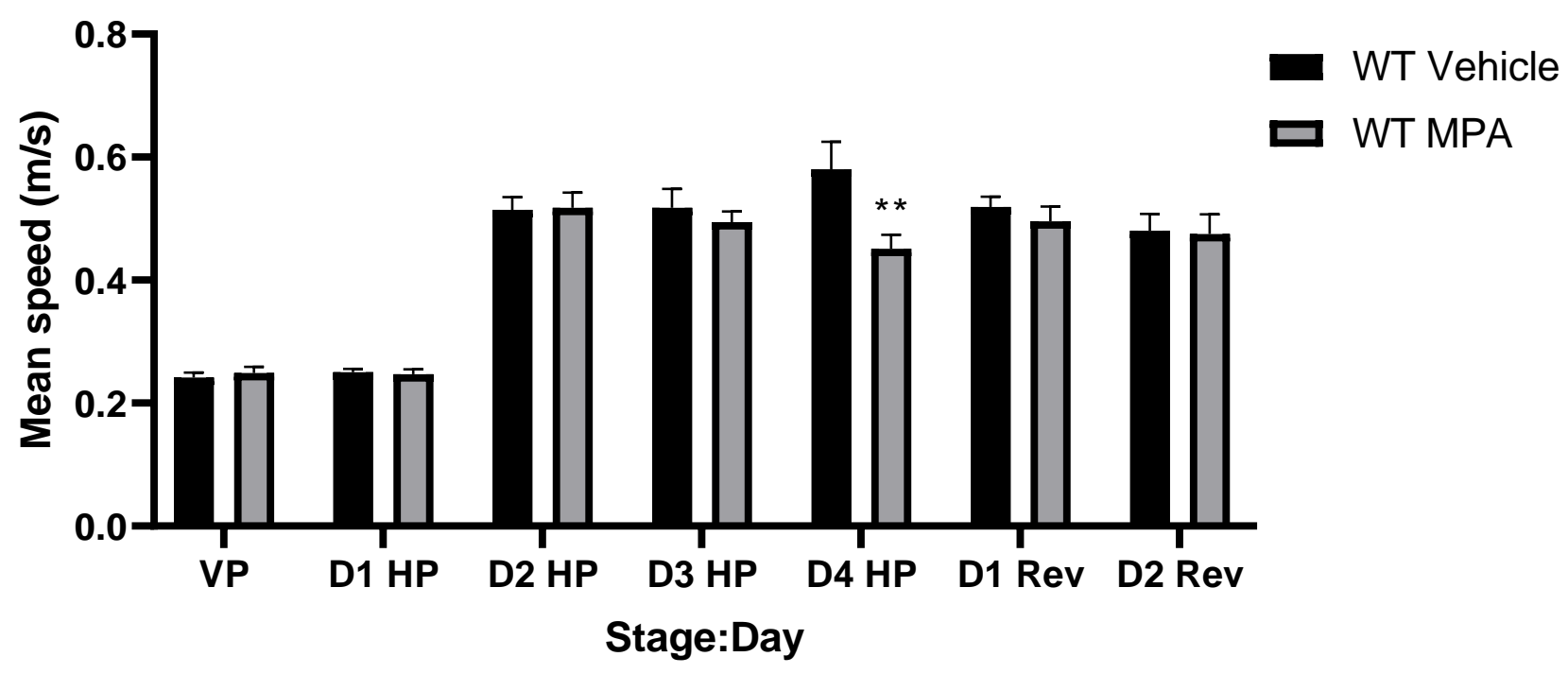

Figure 2. Swim speed and latency to platform in MWM task of WT mice. Mice ( $n=5$ per experimental group) were tested in the MWM. Swim speeds across 5 trials per day were averaged. HP: Hidden Platform, Rev: Reversal. 
A

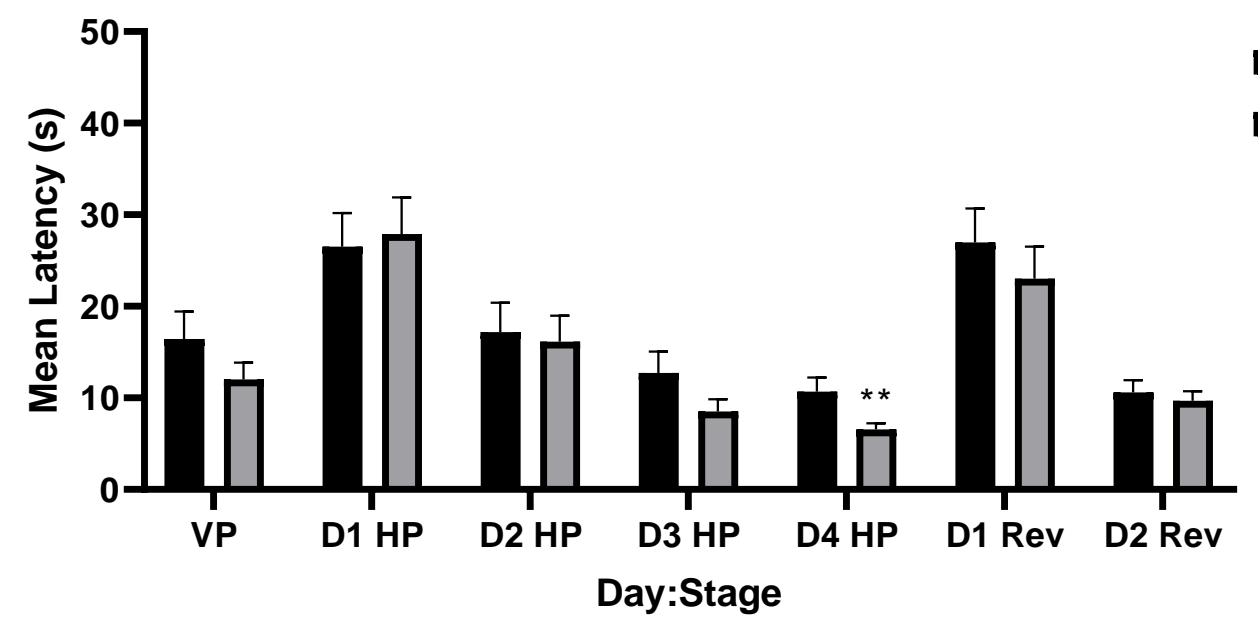

B

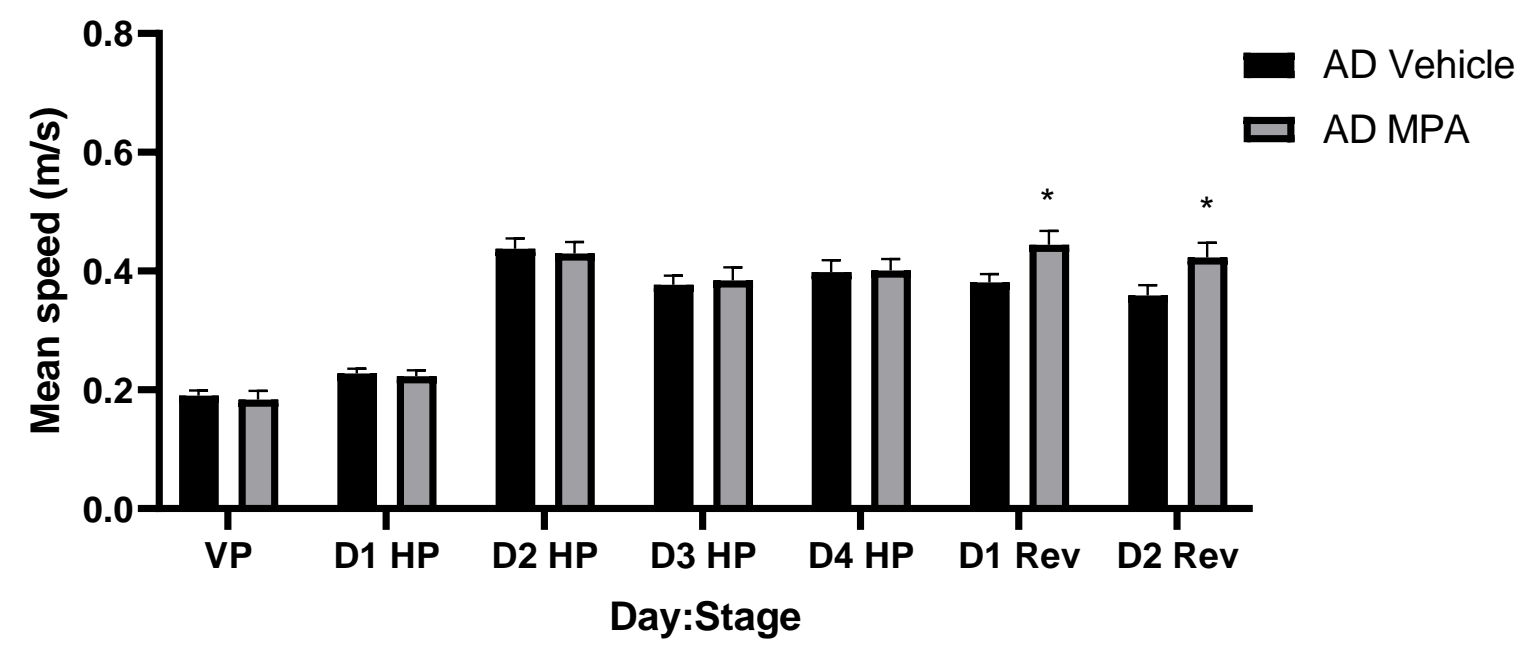

Figure 3. Swim speed and latency to platform in MWM task of AD mice. Mice ( $n=5$ per experimental group) were tested in the MWM. Swim speeds across 5 trials per day were averaged. 
A

Hidden Platform

B
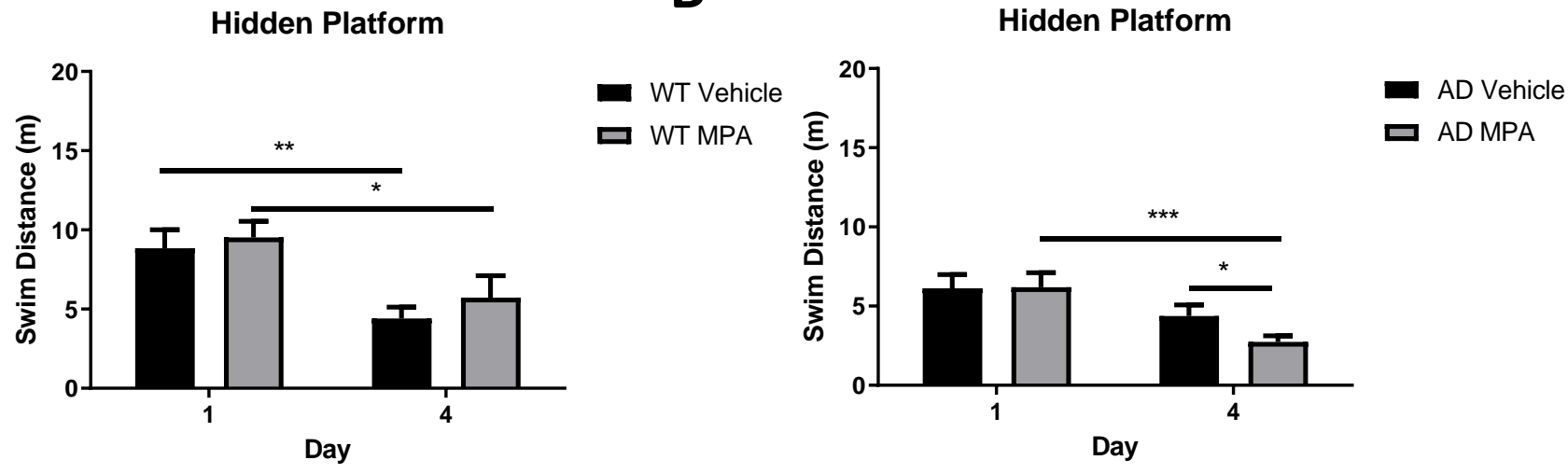

C

Hidden Platform

D

Trial 1
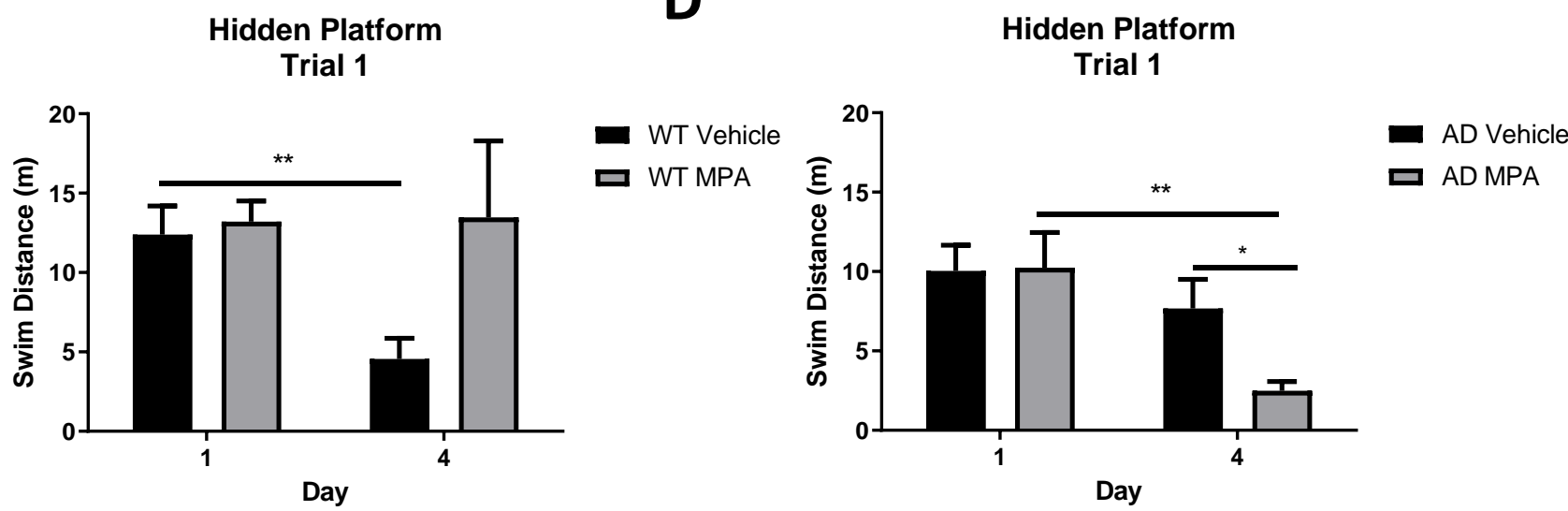

$\mathbf{E}$

$$
\begin{gathered}
\text { Reversal } \\
\text { Day } 1
\end{gathered}
$$

F

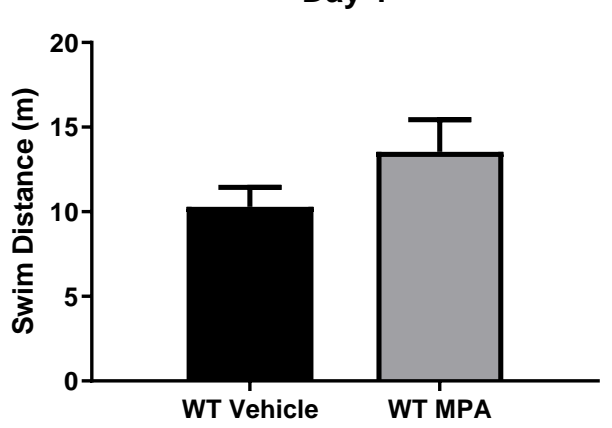

Reversal

Day 1

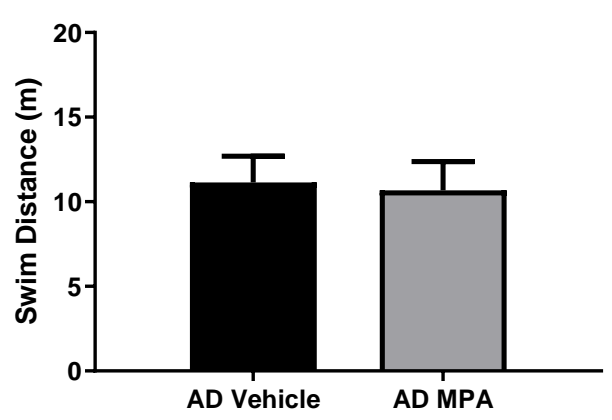

Figure 4. Swim distance in MWM task. Mice ( $n=5$ per experimental group) were tested in the MWM. Mice were trained on the task until optimal performance was maintained for 2 consecutive days, measured by distance swam, before 2 days of reversal training. (A) WT and (B) AD animals' performance the first day of training versus the last day of training (Day 
$1 \vee$ Day 4). (C) WT and (D) AD animals' performance in Trial 1 of the first day of training versus the last day of training (Trial 1 Day $1 \mathrm{v}$ Trial 1 Day 4). (E) WT and (F) AD animals swim distance in reversal training, ${ }^{*} p<0.05, * * p<0.01$, and ${ }^{* * *} p<0.001$, unpaired one-tailed ttest. 

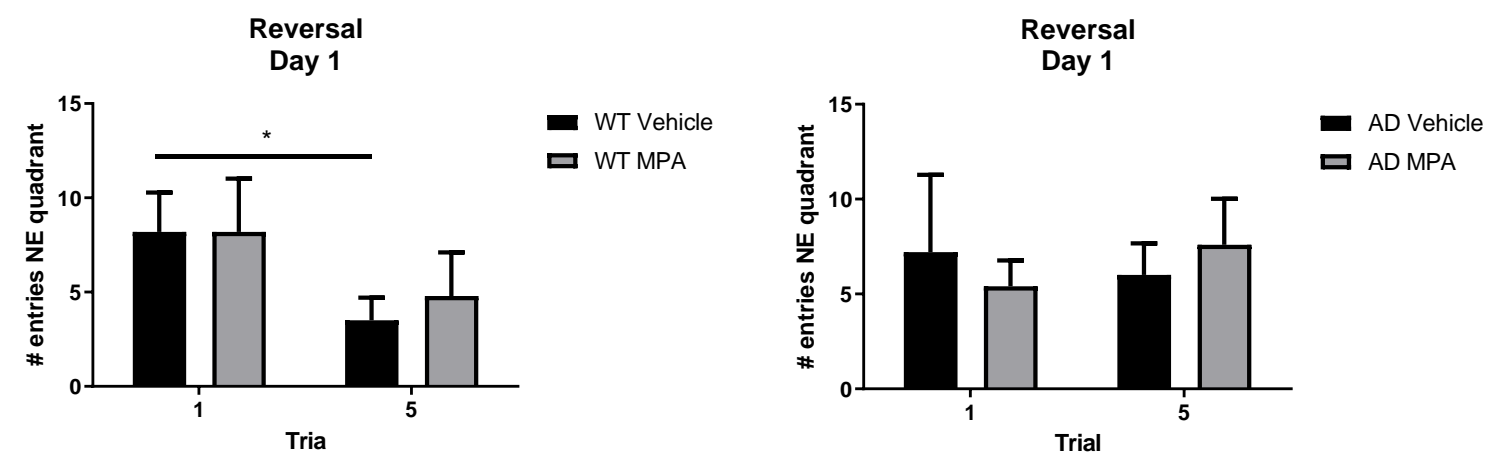

C

Reversal

Day 2 Trial 5

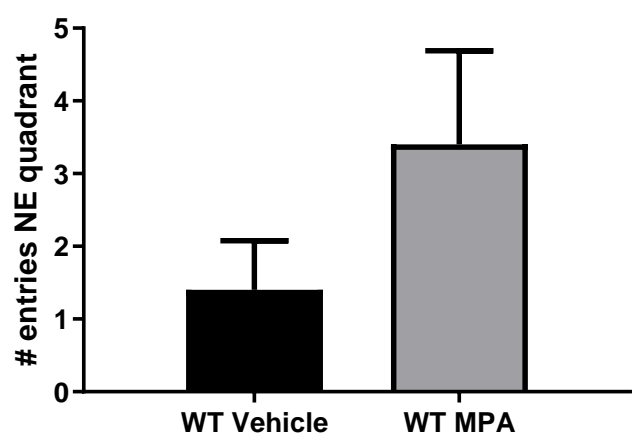

Reversal

Day 2 Trial 5

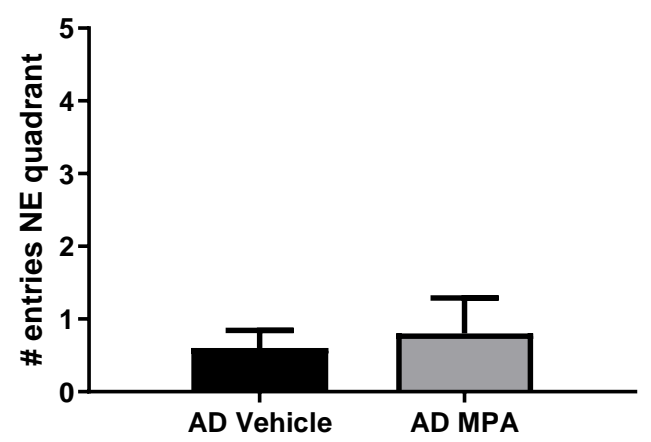

Figure 5. NE quadrant entries during reversal training in MWM task. Mice ( $n=5$ per experimental group) were tested in the MWM. In reversal training, the platform location was changed and the number of entries in the platform's former location (NE) were measured. (A) WT and (B) AD animals' performance the first trial of training versus the last trial of training, on Day 1 (Trial 1 Day 1 v Trial 5 Day 1). (C) WT and (D) AD animals' number of entries into NE on the last trial of all phases of training (last trial of the last day of training; Day 2 Trial 5), *p $<0.05$, unpaired one-tailed t-test. 
A

Cortex

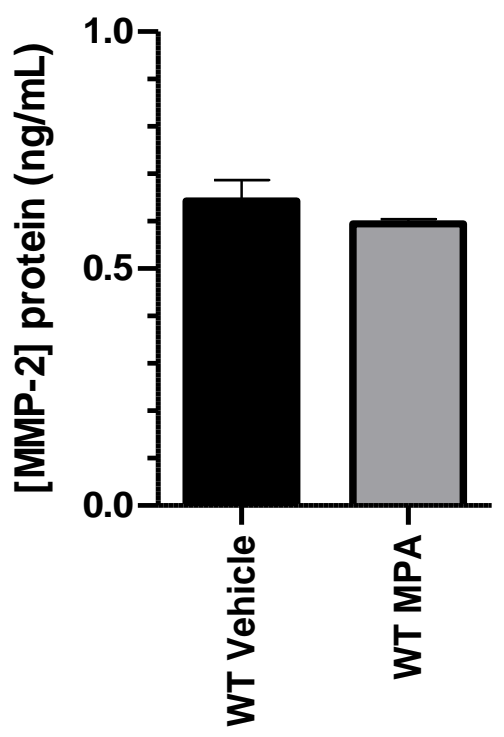

C

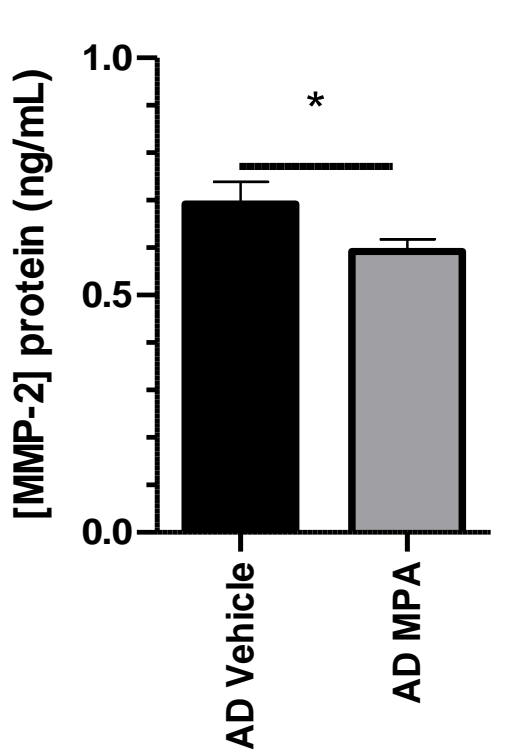

B Hippocampus

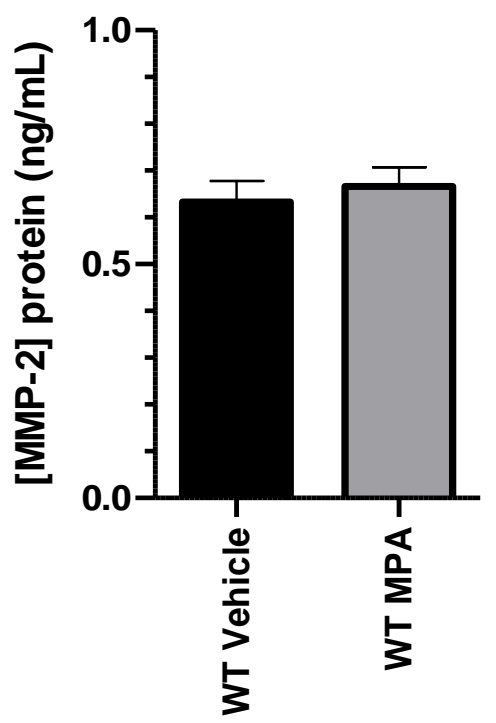

\section{Hippocampus}

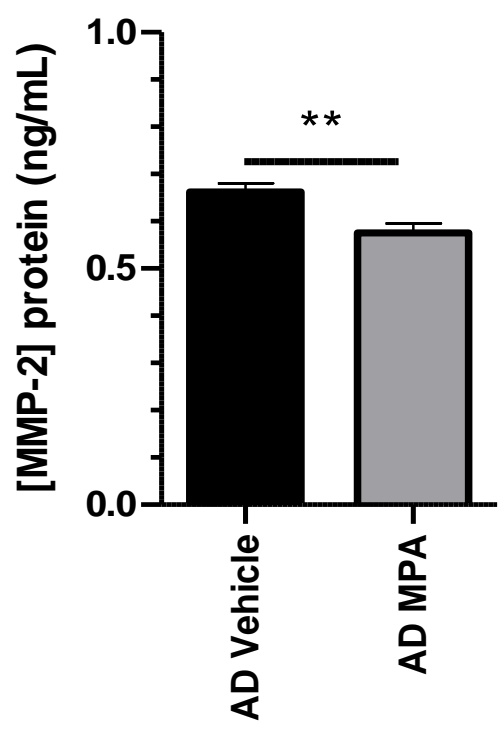

Figure 6. The effect of chronic MPA treatment on MMP-2 expression in the frontal cortex and hippocampus in WT and 3xTg-AD female animals. MMP-2 $(\mathrm{ng} / \mathrm{mL})$ in the specified brain regions was detected using the ELISA kit in WT animals $(A, B)$ and $A D$ animals $(C, D)$ MPAtreated and untreated. MPA treatment had no effects on MMP-2 expression in the frontal cortex of WT animals. MPA treatment significantly decreased MMP-2 in the frontal cortex of 3xTg-AD mice. Results are expressed as means \pm SEM. Results are representative of five animals in each treatment group. ${ }^{*} p<0.05, * * p<0.01$, unpaired one-tailed t-test. 
A

\section{Cortex}

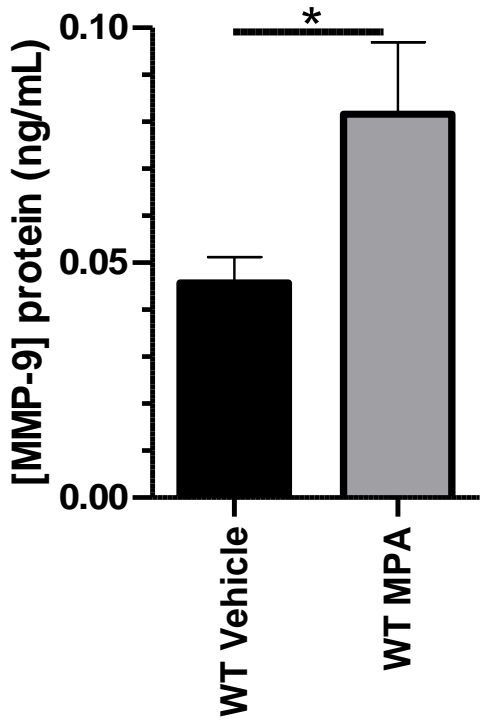

C
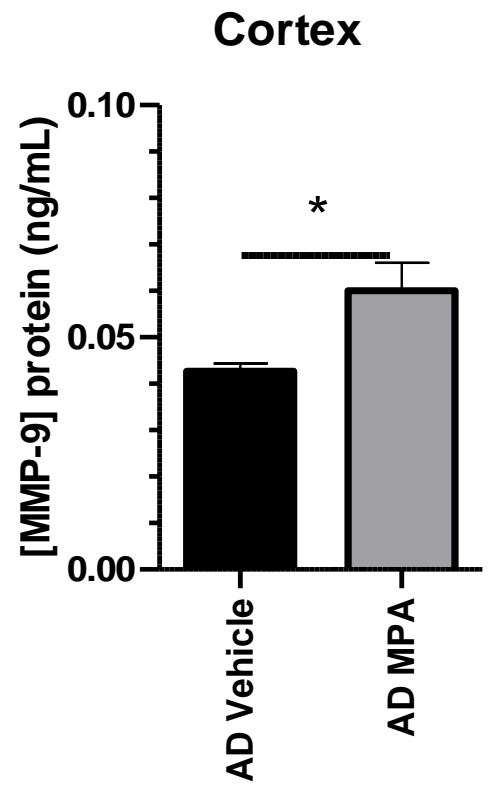

B

Hippocampus

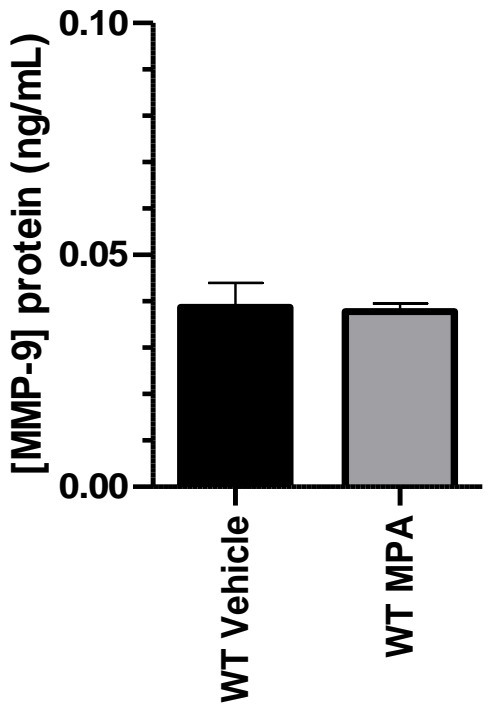

D Hippocampus

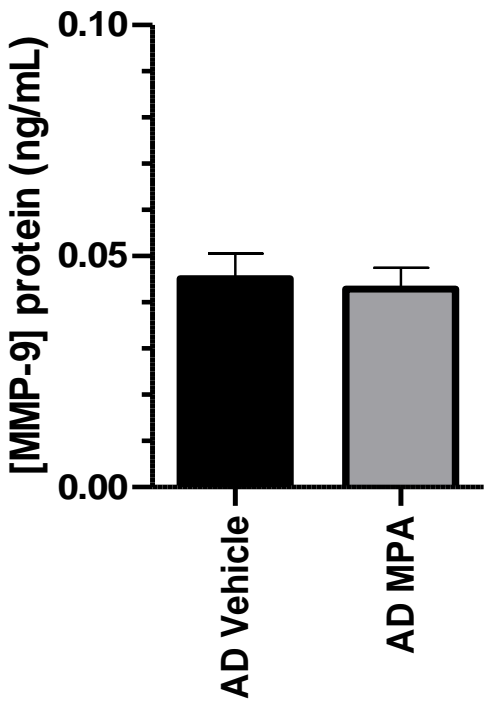

Figure 7. The effect of chronic MPA treatment on MMP-9 expression in the frontal cortex and hippocampus in WT and 3xTg-AD female animals. MMP-9 $(\mathrm{ng} / \mathrm{mL})$ in the specified brain regions was detected using the ELISA kit in WT animals $(A, B)$ and AD animals $(C, D)$ MPAtreated and untreated. MPA treatment had significantly increased MMP-9 expression in the frontal cortex of both WT and AD animals. Results are expressed as means \pm SEM. Results are representative of five animals in each treatment group. ${ }^{*} p<0.05,{ }^{* *} p<0.01$, unpaired one-tailed t-test. 
Chapter 4

General Discussion 
Until the 1980s, women were intentionally excluded from participating in clinical trials. This longtime bias yielded a striking gap in knowledge about women's health and the significant role that sex- and gender-specific differences play in health and disease. The 1993 NIH Revitalization Act required that women be included in all clinical studies and that trials be designed to permit analysis of different effects in women, and in 2016, NIH finally made it an experimental necessity for researchers to factor in sex as a biological variable in animal and human studies, and otherwise required an experimental justification (Mazure et al., 2015). Even though there have been many advances made in women's health research, Alzheimer's disease $(A D)$ is a prime example of a condition for which there is currently limited and superficial understanding of sex differences. Even considering the last decade, sex- and gender-specific differences in AD have been severely understudied. Our ability to address the risks for $A D$ is impeded by the paucity of knowledge regarding how and why the disease differs between men and women. Sex- and gender-specific risk factors are just one of the many areas in which more research into the differences between the sexes in AD is needed. Previous research has often overlooked sex differences in diagnosis, clinical trial design, and treatment outcomes, while also failing to consider the influence of social and environmental factors, in turn, delaying progress in detection and care. In order to optimize the development of current and future interventions in $A D$, these differences cannot be ignored.

The interplay between biological factors (genetic and hormonal influences) and environmental factors is essential to understanding how the risk for AD can be reduced, and how new strategies for prevention and treatment should be developed and implemented. 
Hormonal influences are of interest because there is an extensive amount of literature that suggests menopause and ovariectomy lead to negative cognitive changes and may represent sex-specific risk factors for AD (Pike et al., 2017; Gurvich et al., 2018; Nebel et al., 2018; Rahman et al., 2019). Collectively, sex steroids have been shown to play an important role in the sex differences in the brain and the role of endogenous and exogenous estrogenrelated compounds is well-characterized, yet relatively little is known about the long-term effects of exogenous synthetic progestins on the brain and their role in the pathogenesis of AD. Medroxyprogesterone acetate (MPA) is used by approximately 1 in 5 adolescents and adult women in the United State who are sexually active (Mosher et al., 2010). Globally, nearly 48 million women utilize injectable contraceptives to prevent pregnancy, with the majority of users utilizing MPA as their hormone of choice (United Nations, 2013). Therefore, studies that elucidate the mechanisms and long-term effects of exogenous administration of MPA have the potential to impact millions of women, and further the current mission to promote research on the biological sex differences in disease and improving women's health through science, policy, and education.

Two major studies were designed to begin addressing increasingly relevant knowledge gaps associated with the effects of exogenous progestin treatment. The overall goal of my dissertation project was to investigate the acute and chronic effects of MPA administration on AD-related pathology, in vitro and in vivo, utilizing hormonal modulation of $A \beta$-degrading enzymes, matrix metalloproteinases (MMPs). This chapter includes a discussion of the major findings as related to the literature on MPA, MMPs, and cognitive function, and what implications may be valuable for future epidemiological investigations. 
The chapter concludes with a brief summary and discussion of the limitations of the study, as well as suggestions for future experiments.

This chapter contains discussion and future research possibilities to help answer the research questions:

(R1): What are the outcomes of MPA on MMP-9 secretory, proteolytic, and A $\beta$ degrading activities, in vitro?

(R2): What are the effects of long-term, subcutaneous administration of MPA on cognitive functions and AD-related neuropathology in WT and 3xTg-AD mice?

The central hypothesis of this dissertation was that MPA treatment downregulates MMP-9 expression and subsequent secretion, in turn, disrupting the proteolytic degradation of amyloid beta. Further, the effects on MMP-9 would also negatively impact cognitive function and AD-related neuropathology. The proposed research was based upon the notion that the current literature extensively focuses on the effects of other classes of hormones, with very few studies evaluating the effects of MPA on cognition of women in their reproductive prime, despite evidence that MPA negatively impacts cognitive function in menopausal women and animal models (Braden et al., 2010, 2011, 2017; Gabriel and Fahim et al., 2005; Epperson et al., 2013; Greendale et al., 2009; Espeland et al., 2013; Gleason et al., 2015; Henderson et al., 2016; Manson et al., 2017; Shumaker et al., 2003, 2004).

To address this, I first conducted a survey of what is known about progestins based on the current literature, and how the literature on MPA fell short of characterizing its long- 
term effects in an intact animal model and in younger women. We designed an in vitro exposure model, to determine the impact of MPA on MMP production and activity, and how that may affect amyloid beta degradation. Through this, I was able to show MPA could suppress MMP-9 expression and proteolytic activity, which fell in line with the previously literature, however my experiment was novel in its use of a CNS-related cell line (glial cells). For this study, I used the C6 glial cell line to demonstrate how changes in MMP-9 expression and secretion from glial cells may impact degradation of amyloid beta. We then investigated the consequential effects on amyloid beta degradation and show that MPA impairs the degradation of amyloid beta, in an MMP-9 dependent manner. Furthermore, we revealed MPA's effect on MMP-9 is mediated by the glucocorticoid receptor (GR). The direct impact of this study was to show the potential for MPA to negatively affects cellular processes that are regulated by MMP-9, in vivo, due to the negative impact MPA showed, in vitro. Our ability to easily perform dose-response and time-course studies, in a homogeneous population of cells, presented a highly controlled environment. However, primary cells are more reliable than transformed cell lines. C6 cells have spontaneous NF-kB nuclear activity, suggesting the presence of constitutive NF-KB activity, which explains the constitutive basal expression of MMP-9 observed in our experiments (Robe et al., 2004), and circumvented the necessity for induction or transfection. Considering that we would be required to either induce MMP-9's expression pharmacologically or transfect an MMP-9 expression vector into the primary cells, we decided to perform an in vivo experiment using wild-type and triple transgenic Alzheimer's disease mice, to confirm our observed effects. 
At the molecular level, MPA elicits its biological effects through multiple receptors, including the progesterone receptor (PR), androgen receptor (AR), and glucocorticoid receptor (GR). The hormone binds to the GR with a high affinity, acting as a partial to full agonist for the GR (Koubovec et al., 2004, 2005; Africander et al., 2011; Su et al., 2012b, 2012a; Louw-du Toit et al., 2014). The initial finding of MPA's capability of interfering with the activities of NF-KB or AP-1, at the promoter level, suggested MPA represses cytokineinduced, AP-1 driven genes, as well as NF-KB-driven genes, without impacting the DNAbinding activity of NF-KB, in a GR-dependent manner (Koubovec et al., 2004). We treated C6 cells with a GR antagonist, which attenuated MPA's effects on MMP-9 enzymatic activity (Chapter 2; Figure 4). In this case, our data implicate a GR-dependent transrepression of MMP-9, via interaction with either NF-KB or AP-1, which are generally accepted as regulators of MMP-9 expression (Jonat et al., 1990; Paliogianni et al., 1993; Yokoo and Kitamura, 1996; Barnes, 1998; Bond et al., 2001; Ronacher et al., 2009; Africander et al., 2011; Li et al., 2012; Mittelstadt and Patel, 2012). Therefore, it's plausible the progestin binds to the cytosolic GR, activates the GR, and then activated GR binds to NF-KB/AP-1, in turn, negatively interfering with the transcriptional enhancer and, in turn, promoting transrepression of MMP-9. Future studies should assess MPA's effects on nuclear translocation, utilizing western blot analysis and confocal imaging. Under control conditions, in vitro, $\mathrm{AP}-1$ and $\mathrm{NF}_{k} \mathrm{~B}$ translocate to the nucleus, to bind the promoter region of MMP-9 and, in turn, induce its expression. MPA treatment, in vitro, would hypothetically interfere with this translocation, thus the expression of $\mathrm{AP}-1$ and $\mathrm{NF}_{\mathrm{k}} \mathrm{B}$ subunits, in the nuclear fraction, would decrease, while phosphorylation of proteins in the cytosolic fraction 
would also decrease. This can also be shown with immunofluorescent staining, using antibodies for p65 (transactivator of NFkB), phospho-c-Jun (subunit of AP-1), and c-Fos (subunit of AP-1). Under control conditions, in vitro, staining would be shown in the nucleus, and upon MPA treatment, the staining will be in the cytoplasm. Future studies should aim to further delineate the precise mechanisms by which our observations in the present study occur.

Next, I demonstrated the effects of MPA on cognitive function and brain MMP-9 expression, in vivo. Through this study, I established the correlation between MMP-9 and cognitive function, in intact, female WT and 3x-TgAD mice. The initial goal was to associate reduced MMP-9 expression with cognitive impairments. However, MPA treatment unexpectedly induced MMP-9 expression, in the frontal cortices, of both the WT and AD groups. Additionally, MPA treatment modestly impaired memory consolidation and cognitive flexibility in WT animals, but slightly enhanced memory consolidation in AD animals. The impairment of memory consolidation in the WT animals may be attributed to increased disruption of the perineuronal net (PNN), via the 2-fold increase in MMP-9 expression in the frontal cortex. Notably, with an $\mathrm{n}$ of 5 in each group, our behavior study did not have strong statistical power. Thus, our results serve as preliminary/pilot data and should prompt further elaborate investigation of the effects of chronic MPA on cognitive flexibility and memory consolidation, with an evaluation of the role that the PNN plays in the differential effects we observed between WT and AD animals.

Synthetic glucocorticoids are widely used for treatment of many inflammatory conditions. Generally, hormones which act through the GR have been assumed to be anti- 
inflammatory, however, several studies have shown that there are several factors that allow hormones to exert pro-inflammatory effects. Short-term exposure to glucocorticoids may suppress neuroinflammation, while chronic exposure may promote inflammation in the frontal cortex and hippocampus (Hu et al., 2016). Chapter 2 demonstrated MPA's effects on MMP-9 expression are mediated in a GR-dependent manner. In vitro, MPA caused a dosedependent reduction in MMP-9's expression and enzymatic activity, in turn negatively impacting the degradation of A $\beta$. In vivo, MPA increased MMP-9 protein expression, specifically in the frontal cortex. Depending on dose/concentration, treatment paradigm, the tissue being studied, and the steroid hormone being used, glucocorticoids can promote either anti-inflammatory or pro-inflammatory responses (Dinkel et al., 2002; Cruz-Topete and Cidlowski, 2015; Desmet and De Bosscher, 2017). Additionally, whether proinflammatory GC effects occur in the brain can depend upon the use of synthetic versus endogenous glucocorticoids and the timing of exposure with respect to the inflammatory challenge, but unfortunately the precise mechanism(s) by which the same hormone can promote two opposing responses are not well understood. The discrepancy between our in vitro and in vivo studies are most likely attributed to the differences in treatment paradigms, where our in vitro treatments were relatively acute treatments and our in vivo treatment was a chronic, slow-release depot of the hormone. We expected there would be a cognitive impairment that would be exacerbated with MPA treatment, but in fact, we observed improvement. Moderate levels of glucocorticoids have been shown to enhance both long-term potentiation (LTP) and memory consolidation, while very low or very high levels inhibit these processes (Joels and Krugers, 2007; Wirth et al., 2015). Additionally, too 
little or too much receptor occupancy leads to an impairment as well (de Kloet et al., 1999). Therefore, it is plausible that our chronic MPA treatment alters GR expression. In theory, with a lower number of receptors, the receptor can become saturated at lower circulating levels of hormone. We suspect that as a result of our treatment, the AD animals possessed a higher GR expression when compared to the WT-treated animals. Given the clinical implications of the pro-inflammatory effects of MPA, it is crucial for future investigations to focus on the capacity of MPA to increase CNS inflammation and alter GR expression.

Our in vivo study allowed for the flexibility to assess the effects of MPA, in two genotypes of mice that are genetically homogenous. The dosage of MPA used was standard dosage form that produced a pseudo pregnancy state and was administered at a controlled dosing interval. Additionally, we were able to perform behavior assessments of the animals and follow these assessments with biochemical analyses. Our data suggests aging the animals to 6-months of age did not show a significant cognitive impairment. The effects of MPA on the GR in brain structures involved in the stress response should also be studied. As previously mentioned, hormones exert negative feedback on the HPA axis, through their actions on the endometrium. Studying the effects of MPA on the HPA axis could determine how the GR adapts after chronic MPA treatment, as well as determine if the MWM behavior task itself promotes any alterations in GR expression. This would determine if MPA impairs or suppressed the ability of the animals to respond to stress, including stress that may have been a byproduct of performing in the MWM task. Future studies should further investigate the effects of chronic MPA treatment on GR expression. 
Obviously, mice are not human beings, thus we cannot be aware of the extent that the data would translate into the clinic. We also were forced to make an informed guess as to when cognitive decline would present itself and we seemingly did not observe the mutation-induced cognitive decline in the $A D$ animals. We also selected a single dose and a single dosing regimen. A major limitation of our in vivo study was the number of mice per group, which limited our ability to interpret the behavior data. Our protein measurements show increased MMP-9 expression after MPA treatment. However, the specific cell type(s) this effect is attributed to remains to be determined. Immunofluorescence could be used to identify the cell types where increased MMP-9 expression is located. Although we were able to measure protein, we were unable to make the correlation of protein with enzymatic activity, due to our inability to detect it via zymography. Future studies could assess MMP-9 activity in histological sections via in situ zymography, where frozen sections are coated with fluorescently labeled matrix proteins, incubated, and observed for black patches in the fluorescent background, indicating proteolysis of the matrix protein (George and Johnson, 2010). Alternatively, frozen sections could also be incubated with dye-quenched (DQ) gelatin, which would become fluorescent upon proteolytic cleavage by MMP-9. In this method, MMP-9 activity would be denoted as bright fluorescent spots (Gawlak et al., 2009). Additionally, Oddo et al. reported that at the 6-month timepoint, in 3xTg-AD mice, synaptic dysfunction and LTP deficits are observed, but extracellular A $\beta$ deposits are not in the hippocampus, even though intracellular $A \beta$ immunoreactivity is apparent at this age (2003). We were also unable to achieve detectable levels of amyloid beta in our detergent-soluble samples, of both WT and AD mice. Once we revisited the literature, we found that $A \beta_{42}$ is 
significantly increased at 6 months of age, compared to animals at 2 months, in the detergent-insoluble fraction, the form of $A \beta$ that readily forms aggregates (Billings et al., 2005). Therefore, future studies should prioritize the insoluble fraction of the cortex, and assay for $A \beta_{42}$. Inconsistencies within the literature are most likely due to variability in the method chosen to quantify $A \beta$, which ultimately influences the species you're able to analyze. Additionally, it is plausible that the behavior study itself altered endogenous glucocorticoid release and receptor expression, influencing the responses to MPA treatment. Future studies should aim to assess the effects of chronic MPA treatment on MMPs in Morris Water Maze-trained and -untrained mice. Such studies could provide insight into the role of education (training that is sometimes stressful in humans) and its association with decreased risk for Alzheimer's disease. We suggest a continuation of the experiments we performed, where there are multiple age groups (3-, 6-, 9-, 12-month), multiple hormone treatment regimens (acute and chronic), an assessment of detergent-insoluble $A \beta_{40}$ and $A \beta_{42}$, and a battery of behavior assessments, in order to fully assess the effects of MPA on learning and memory.

My studies on the acute and chronic effects of MPA have confirmed this progestin can modulate the levels of MMP-9, in a CNS-related system, both in vitro and in vivo, as well as differentially impact cognitive function in intact, female WT and 3xTg-AD mice. In summary, our in vitro data suggests MPA: (a) downregulates MMP-9 expression and production, (b) impairs the neuroprotective effect of the proteolytic cleavage of toxic $A \beta_{1-42}$ species, and (c) suppresses the transcription of glial MMP-9, through the glucocorticoid receptor. In vivo, MPA: (a) enhances memory consolidation and working memory in AD mice, (b) impairs memory consolidation in WT mice, and (c) increases MMP-9 levels in the 
frontal cortices of both WT and AD mice. Collectively, these findings indicate MPA's

differential effects should be further investigated, along with the underlying mechanisms regarding the signaling mechanisms behind our in vitro and in vivo observations.

\section{Future Directions \& Conclusions}

There are specific areas within the sex- and gender-specific risk factors in AD that are currently understudied and must be of priority, due to their direct impact on the future progress of the field. Prior to our work, there weren't many studies that assessed how synthetic progestins influences cognition and AD risk (Braden et al., 2011; Olanrewaju et al., 2013). My work provides indications of acute versus chronic administration of MPA, the effects on MMP-9 expression, and the impacts on cognition and AD-related pathology. Additional investigations must follow these initial studies, as well as studies addressing how synthetic progestins might influence AD biomarkers, in addition to their effects on MMP-9. The field also accepts that AD presents itself more severely and progresses faster in woman more than it does in men, thus the reasoning behind why women are more vulnerable should also be investigated (Barnes et al., 2005; Hua et al., 2010; Holland et al., 2013; Lin et al., 2015; Filon et al., 2016; Koran et al., 2017). Moving forward, when evaluating the effects of exogenous hormones, particularly hormonal contraception, researchers should improve the details they include, regarding the specific type of contraception evaluated (estrogencontaining versus progestin-only). The lack of information regarding the long-term effects of contraceptives, in general, is one of the main rationales behind the Nurses' Health Studies (I and II). In both cases, and even in a follow-up study that occurred in 2014, whether the oral contraceptive was a combination pill versus a progestin-only pill was not recorded; they 
were either "Never" or "Ever" users (Charlton et al., 2014). Due to the neuroprotective actions of estrogen-related compounds, this convolutes how we interpret those data. They also recorded HT use and noted there were missing data points, demonstrating not only are their gaps in knowledge, but also gaps in data.

Menopause is associated with fluctuation of endogenous hormone levels, as well as increased risk of AD in women (Fisher et al., 2018). Thus, we understand why majority of the literature has focused on menopausal influence on $A D$ and the effects of exogenous hormones on AD risk. However, moving forward, researchers should be more inclusive of younger, intact (non-menopausal) pre-clinical and clinical models, when evaluating the long-term effects of exogenous hormones. Pike et al., performed 3 individual experiments, evaluating the effects of estrogen and progesterone on AD-related pathology (2007). In their first experiment, they used intact female 3xTg-AD mice to study the development of $A D$ neuropathology as an effect of age. However, in the remainder of their experiments, they used OVX animals and ovariectomy exacerbated $A \beta$ accumulation and tau hyperphosphorylation. Estrogen treatment provided neuroprotection from these pathologies, but combined estrogen and progesterone treatment attenuated this effect. Interestingly, progesterone alone treatment yielded no effect on the accumulation of $A \beta$, but successfully reduced tau hyperphosphorylation, unlike estrogen. It is unclear why their hormone treated groups only included OVX female 3xTg-AD animals and failed to include gonadally intact animals. Oftentimes, researchers use OVX animal models, to reduce variability in their outcome measures, however, the estrous cycle is essentially suspended once the subjects are chronically treated with hormonal contraceptives, thus variability 
should not be much of a concern. Nonetheless, our findings in our AD animals are, in part, supported by the conflicting literature, although they utilized postmenopausal models (Paganini-Hill and Henderson, 1996; Tang et al.,1996; Kawas et al., 1997; Hogervorst et al., 2000; Zandi et al, 2002; Maki et al., 2005; Pike et al., 2017). Our contribution to the field is one of the first investigations of the effects of this specific progestin, in an intact WT and AD model.

Not only are women victims to an increased AD burden, but they also have an increased stroke burden, thus there is an additional, urgent need to understand the risk factors, in stroke, that are unique to women. The vast majority of strokes occur in men and women over the age of 65 , however nearly $15 \%$ of all strokes affect individuals age 45 and younger. Additionally, the narrow focus on the effects of estrogen-related compounds, and paucity of research addressing the potential effects of synthetic progestin compounds, in non-menopausal models, transcends beyond the AD field. The neuroprotective efficacy of estrogen-related compounds is well-established in the stroke field. Thus, the findings from this project can also be applied to acute neurological disease conditions, like stroke, and promote further investigation of the effects of synthetic progestins on post-stroke outcomes.

Many studies have demonstrated that the upregulation of MMPs, specifically MMP9 levels post-stroke, is strongly correlated with blood-brain barrier (BBB) disruption, increased infarct volumes, and poor post-stroke outcomes (Asahi et al., 2000; Asahi et al., 2001; Rosell et al., 2005; Sotgiu et al., 2006; Vukasovic et al., 2006; Moldes et al., 2008; Sandoval and Witt et al., 2008; Hu et al., 2009; Wang et al., 2009; Li et al., 2013). 
Additionally, the literature suggests MMP-9 inhibition is neuroprotective against changes in BBB permeability (Svedin et al., 2007) and highlights the prominent role MMP-9 plays at the BBB post-stroke (Dejonckheere et al., 2011). MMP-9 knockout animals have been shown to have improved post-stroke outcomes (reductions in infarct volume, cerebral edema, and functional deficits). Clinical data also reveals that MMP-9 is increased following stroke in humans and is strongly correlated with worse post-stroke outcomes (Montaner et al., 2001; Copin et al., 2005; Ning et al., 2006; Lucivero et al., 2007; Rosell et al., 2008). Regarding the mechanism by which these effects occurs, the field debates over whether resident brain cells, circulating immune cells, or cells within the vasculature are responsible for the increased expression of MMP-9 post-stroke. Turner et al., (2016) hypothesizes that neutrophils are primary source of MMP-9 and once ischemic injury occurs, MMP-9 transmigrates into the tissue and, in turn, degrades the ECM and disrupts the BBB, consequently promoting the secretion of MMP-9 from neurons and microglia. The precise mechanism must be further elucidated.

Of interest, is the disparity between men and women in disability and recovery following a stroke. According to the Framingham Heart Study, women were more vulnerable to more severe post-stroke outcomes (Petrea et al., 2009). Even though women are known to experience more debilitating strokes and suffer from a lower quality of life post-stroke (Gargano et al., 2007; Martínez-Sánchez et al., 2011a,b), previous research falls short of addressing post-stroke outcomes prior to menopause and fail to specifically evaluate the effects of MPA on post-stroke outcomes, during a woman's reproductive prime. For example, the Women's Health Initiative (WHI) suggests that oral therapy with 
combined equine estrogen (CEE) alone or in combination with MPA (CEE/MPA) are risk factors for ischemic stroke in healthy postmenopausal women (Wassertheil-Smoller et al., 2003; Anderson et al., 2004). Littleton-Kearney et al., evaluated the consequences of longterm oral administration of CEE alone or in combination with MPA on infarct volumes and observed reduced cortical lesion volume after 2-months of treating OVX rats with either CEE or CEE/MPA. In Chapter 3, we found that MPA promoted nearly a 2-fold increase of MMP-9 protein levels in the cortex of our WT animals. Theoretically, if the baseline level of MMP-9 is already elevated prior to an ischemic insult, relative to control, this would exacerbate the deleterious effects of MMP-9, post-stroke. The literature states baseline differences between men and women (age, comorbidities, severity, and pre-stroke disability) explains the increased mortality rate in women, thus hormone use could additionally impact poststroke outcomes. Future research should aim to determine whether MPA worsens poststroke outcomes, via the upregulation of MMP-9.

Overall, my dissertation project compliments the existing literature which highlights MPA's limited neuroprotective efficacy. Additionally, my dissertation project suggests there may be additional exogenous hormone treatments that, when chronically used, have adverse effects on the brain. The more we understand the long-term effects of progestins on the brain, the more researchers can address the critical knowledge gaps in the literature, and further elucidate if exogenous hormones pose a sex-specific risk factor for cognitive dysfunctions in women. Due to the discrepancies and knowledge gaps within the pre-clinical and clinical literature, further work on the influence of synthetic progestins on brain function and $A D$ risk is warranted as a potential sex-specific risk factor until a consensus is 
reached. However, we are hoping our work serves as a pilot study to increase awareness and merit further exploration. Until we elaborately examine the influence of exogenous hormones on the sex and gender differences within $A D$, the field will never be as productive as it has the potential to be. Addressing the differences between women and men benefits everyone. Proper diagnosis becomes difficult due to differences in symptoms, while management of the disease become challenging due to differences in the responses to treatment. By focusing on sex- and gender-specific differences and developing appropriate strategies for clinical studies, we can continue to fill the current knowledge gaps in the field, ultimately improving health outcomes for both women and men. 


\section{References}

Abdul-Muneer, P.M., Schuetz H., Wang F., Skotak M., Jones J., Gorantla S., Zimmerman M.C., Chandra N., Haorah J. (2013) Induction of oxidative and nitrosative damage leads to cerebrovascular inflammation in an animal model of mild traumatic brain injury induced by primary blast. Free Radic. Biol. Med. 60:282-291.

Africander, D., Verhoog, N., and Hapgood, J. P. (2011). Molecular mechanisms of steroid receptormediated actions by synthetic progestins used in HRT and contraception. Steroids 76, 636652. doi: 10.1016/j.steroids.2011.03.001

Agapova OA, Ricard CS, Salvador-Silva M, Hernandez MR (2001) Expression of matrix metalloproteinases and tissue inhibitors of metalloproteinases in human optic nerve head astrocytes. Glia 33: 205-216.

Akiyama, H., Barger, S., Barnum, S., Bradt, B., Bauer, J., Cole, G. M., et al. (2000). Inflammation and Alzheimer's disease. Neurobiol. Aging 21, 383-421. doi: 10.1016/s0197-4580(00)00124-x

Allen, T. K., Nazzal, M. N., Feng, L., Buhimschi, I. A., and Murtha, A. P. (2019). Progestins inhibit tumor necrosis factor $\alpha$-induced matrix metalloproteinase 9 activity via the glucocorticoid receptor in primary amnion epithelial cells. Reprod. Sci. 26, 1193-1202. doi: $10.1177 / 1933719118811646$

Almeida, O.P., Waterreus, A., Spry, N., Flicker, L., Martins, R.N. (2004). One year follow-up study of the association between chemical castration, sex hormones, beta-amyloid, memory, and depression in men. Psychoneuroendocrinology.29:1071-1081.

Almkvist, O., Basun, H., Wagner, S.L., Rowe, B.A., Wahlund, L.O., Lannfelt, L. (1997). Cerebrospinal fluid levels of alpha-secretase-cleaved soluble amyloid precursor protein mirror cognition in a Swedish family with Alzheimer disease and a gene mutation. Arch. Neurol. 54, 641-644. 
Altmann, A., Tian, L., Henderson, V.W. et al. (2014) Sex modifies the APOE-related risk of developing Alzheimer disease. Ann Neurol 75, 563-573.

Alzheimer's A (2019) 2019 Alzheimer's disease facts and figures. Alzheimers Dement 14, 367-429.

Amtul, Z., Uhrig, M., Supino, R., Beyreuther, K. (2010). Phospholipids and a phospholipid-rich diet alter the in vitro amyloid-beta peptide levels and amyloid-beta 42/40 ratios. Neurosci. Lett. 481: 73-77.

Anderson, G.L., Limacher, M., Assaf, A.R., Bassford, T., Beresford, S.A., Black, H., Bonds, D, et al., (2004) Women's Health Initiative Steering Committee. Effects of conjugated equine estrogen in postmenopausal women with hysterectomy: the Women's Health Initiative randomized controlled trial. JAMA. Apr 14;291(14):1701-12. PubMed 15082697

Anderson, J.J., Holtz, G., Wang, R., Mazzarelli, L., Wagner, S.L., Menzaghi, F. (1999). Reduced cerebrospinal fluid levels of alpha-secretase-cleaved amyloid precursor protein in aged rats: correlation with spatial memory deficits. Neuroscience. 93:1409-1420.

Arriagada, P.V., Growdon, J.H., Hedley-Whyte, E.T., Hyman, B.T. (1992). Neurofibrillary tangles but not senile plaques para llel duration and severity of Alzheimer's disease. Neurology 42:6319.

Asahi, M., Asahi, K., Jung, J.C., del Zoppo, G.J., Fini, M.E., Lo, E.H. (2000) Role of matrix metalloproteinase 9 in focal cerebral ischemia: effects of gene knockout and enzyme inhibition with BB-94. J Cereb Blood Flow Metab. 20:1681-1690. 
Asahi, M., Wang, X., Mori, T., Sumii, T., Jung, J.C. et al (2001) Effects of Matrix Metalloproteinase-9 Gene Knock-Out on the Proteolysis of Blood-Brain Barrier and White Matter Components after Cerebral Ischemia. J Neurosci. 21:7724-7732.

Asahina, M., Yoshiyama, Y., and Hattori, T. (2001) Expression of matrix metalloproteinase-9 and urinary-type plasminogen activator in Alzheimer's disease brain. Clin. Neuropathol. 20, 6063.

Augustin, R., Endres, K., Reinhardt, S., Kuhn, P.H., Lichtenthaler, S.F., Hansen, J., et al. (2012) Computational identification and experimental validation of microRNAs binding to the Alzheimer-related gene ADAM10. BMC Med Genet 13: 35.

Aujla, P.K., and Huntley, G.W. (2014). Early postnatal expression and localization of matrix metalloproteinases-2 and -9 during establishment of rat hippocampal synaptic circuitry: MMP proteolysis during hippocampal development. J. Comp. Neurol. 522, 1249-1263. doi: 10.1002/cne. 23468

Backstrom, J. R., Lim, G. P., Cullen, M. J., and Tökés, Z. A. (1996). Matrix metalloproteinase-9 (MMP9) Is synthesized in neurons of the human hippocampus and is capable of degrading the amyloid-peptide (1-40). J. Neurosci. 16, 7910-7919. doi: 10.1523/JNEUROSCI.16-2407910.1996

Baddeley, A. D., Bressi, S., Della Sala, S., Logie, R. \& Spinnler, H. (1991) The decline of working memory in Alzheimer's disease: a longitudinal study. Brain 114, 2521-2542.

Banks, W.A. (2012). Brain Meets Body: The Blood-Brain Barrier as an Endocrine Interface. Endocrinology. 153:4111-9. 
Barkus, C., McHugh, S.B., Sprengel, R., Seeburg, P.H., Rawlins, J.N.P., and Bannerman, D.M. (2010) Hippocampal NMDA receptors and anxiety: at the interface between cognition and emotion. European Journal of Pharmacology. 626:49-56.

Barnes, L.L., Wilson, R.S., Bienias, J.L., Schneider, J.A., Evans, D.A., Bennett, D.A. (2005). Sex differences in the clinical manifestations of Alzheimer disease pathology. Arch Gen Psychiatry. 62:685-91

Barnes, P. J. (1998). Anti-inflammatory actions of glucocorticoids: molecular mechanisms. Clin. Sci. 94, 557-572. doi: 10.1042/cs0940557

Bateman, R.J., Xiong, C., Benzinger, T.L., Fagan, A.M., Goate, A., Fox, N.C., Marcus, D.S., Cairns, N.J., Xie, X., Blazey, T.M., Holtzman, D.M., Santacruz, A., Buckles, V., Oliver, A., Moulder, K., Aisen, P.S., Ghetti, B., Klunk, W.E., McDade, E., Martins, R.N., et al. (2012). Clinical and biomarker changes in dominantly inherited Alzheimer's disease. N Engl J Med. 367:795-804. doi: $10.1056 /$ NEJMoa1202753

Bednarek, N., Clément, Y., Lelièvre, V., Olivier, P., Loron, G., Garnotel, R., et al. (2009). Ontogeny of MMPs and TIMPs in the murine neocortex. Pediatr. Res. 65, 296-300. doi: 10.1203/PDR.0b013e3181973aee

Benson, D. L., and Huntley, G. W. (2012). Building and remodeling synapses. Hippocampus 22, 954968. doi: 10.1002/hipo.20872

Berne and Levy Physiology, $7^{\text {th }}$ edition (2017, Elsevier) 
Bernstein, S. L., Dupuis, N.F., Lazo, N.D., Wyttenbach, T., Condron, M.M., Bitan, G. et al. (2009). Amyloid- $\beta$ protein oligomerization and the importance of tetramers and dodecamers in the etiology of Alzheimer's disease. Nature Chem. 1:326-331.

Billings, L.M., Oddo, S., Green, K.N., McGaugh, J.L., Laferla, F.M. (2005) Intraneuronal A $\beta$ causes the onset of early Alzheimer's disease-related cognitive deficits in transgenic mice. Neuron. 45:675-688.

Bilousova, T.V., Dansie, L., Ngo, M., Aye, J., Charles, J.R., Ethell, D.W., et al. (2009). Minocycline promotes dendritic spine maturation and improves behavioural performance in the fragile $X$ mouse model. J. Med. Genet. 46, 94-102. doi: 10.1136/jmg.2008.061796

Binder, L.I., Frankfurter, A., Rebhun, L.I. (1985). The distribution of tau in the mammalian central nervous system. J Cell Biol. 101:1371-8.

Birrell, J.M., and Brown, V.J. (2000). Medial frontal cortex mediates perceptual attentional set shifting in the rat. J. Neurosci. 20, 4320-4324. doi: 10.1523/JNEUROSCI.20-11-04320.2000

Bissonette, G.B., Martins, G.J., Franz, T.M., Harper, E.S., Schoenbaum, G., Powell, E.M. (2008) Double dissociation of the effects of medial and orbital prefrontal cortical lesions on attentional and affective shifts in mice. The Journal of Neuroscience: the Official Journal of the Society for Neuroscience, 28:11124-11130

Bissonette, G.B., Powell, E.M., and Roesch, M.R. (2013). Neural structures underlying set-shifting: roles of medial prefrontal cortex and anterior cingulate cortex. Behav. Brain Res. 250, 91101. doi: 10.1016/j.bbr.2013.04.037 
Block, A.E., Dhanji, H., Thompson-Tardif, S.F., and Floresco, S.B. (2007). Thalamic-prefrontal cortical-ventral striatal circuitry mediates dissociable components of strategy set shifting. Cereb. Cortex 17, 1625-1636. doi: 10.1093/cercor/bhl073

Blondell, S.J., Hammersley-Mather, R., Veerman, J.L. (2014). Does physical activity prevent cognitive decline and dementia?: A systematic review and meta-analysis of longitudinal studies. BMC Public Health 14, 510.

Bloom GS. (2014). Amyloid- $\beta$ and tau: the trigger and bullet in Alzheimer disease pathogenesis. JAMA Neurol 71: 505-8.

Bond, M., Chase, AJ., Baker, AH., and Newby, AC. (2001). Inhibition of transcription factor NF-kB reduces matrix metalloproteinase $-1,-3$ and -9 production by vascular smooth muscle cells. Cardiovasc. Res. 50, 556-565. doi: 10.1016/s0008-6363(01)00220-6

Boswell, H.B., (2014). Normal Pubertal Physiology in Females. pp.7-30.

Bozdagi, O., Nagy, V., Kwei, K.T., Huntley, G.W. (2007). In vivo roles for matrix metalloproteinase-9 in mature hippocampal synaptic physiology and plasticity. J. Neurophysiol. 98, 334-344.

Bozzelli, L.P., Alaiyed, S., Kim, E. et al (2018). Proteolytic remodeling of perineuronal nets: effects on synaptic plasticity and neuronal population dynamics. Neural Plast 2018:1-13. doi:10.1155/2018/5735789

Braden, B., Andrews, M. G., Acosta, J. I., Mennenga, S. E., Lavery, C., and Bimonte-Nelson, H. A. (2017). A comparison of progestins within three classes: differential effects on learning and memory in the aging surgically menopausal rat. Behav. Brain Res. 322, 258-268. doi: 10.1016/j.bbr.2016.06.053 
Braden, B., Garcia, A. N., Mennenga, S. E., Prokai, L., Villa, S. R., Acosta, J. I., et al. (2011). Cognitiveimpairing effects of medroxyprogesterone acetate in the rat: independent and interactive effects across time NIH Public Access. Psychopharmacology 218, 405-418. doi: $10.1007 / \mathrm{s} 00213-011-2322-4$

Braden, B., Talboom, J. S., Crain, I. D., Simard, A. R., Lukas, R. J., Prokai, L., et al. (2010). Medroxyprogesterone acetate impairs memory and alters the GABAergic system in aged surgically menopausal rats. Neurobiol. Learn. Mem. 93, 444-453. doi: 10.1016/j.nlm.2010.01.002

Brannvall K, et al. (2005). 19-Nortestosterone influences neural stem cell proliferation and neurogenesis in the rat brain. Eur J Neurosci. 21:871-8. [PubMed: 15787693]

Brinton, R.D., Thompson, R.F., Foy, M.R., Baudry, M., Wang, J., Finch, C.E., Morgan, T.E., Pike, C.J., Mack, W.J., Stanczyk, F.Z., Nilsen, J. (2008) Progesterone receptors: form and function in brain. Front. Neuroendocrinol., 29:313-339.

Brugger, P., Monsch, A. U., Salmon, D. P., and Butters, N. (1996). Random number generation in dementia of the Alzheimer type: A test of frontal executive functions. Neuropsychologia 34: 97-103

Buchanan, C. D., Mahesh, V. B., and Brann, D. W. (2000). Estrogen-astrocyte-luteinizing hormonereleasing hormone signaling: a role for transforming growth factor- $\beta 11$. Biol. Reprod. 62, 1710-1721. doi: 10.1095/biolreprod62.6.1710

Burns, A., Iliffe, S. (2009). Dementia. BMJ. 338: b75.

Butchart, J., Birch, B., Bassily, R., Wolfe, L., Holmes, C. (2013). Male sex hormones and systemic inflammation in Alzheimer disease. Alzheimer Dis Assoc Disord. 27:153-6. 
Cagnin, A., Brooks, D.J., Kennedy, A.M., Gunn, R.N., Myers, R., Turkheimer, F.E., et al. (2001). Invivo measurement of activated microglia in dementia. Lancet. 358:461 - 7.

Candelario-Jalil, E., Yang, Y., Rosenberg, G.A. (2009) Diverse roles of matrix metalloproteinases and tissue inhibitors of metalloproteinases in neuroinflammation and cerebral ischemia. Neuroscience 158, 983-994.

Cao, J., Drews, M., Lee, H.M., Conner, C., Bahou, W.F., Zucker, S. (1998). The propeptide domain of membrane type 1 matrix metalloproteinase is required for binding of tissue inhibitor of metalloproteinases and for activation of pro-gelatinase A. J Biol Chem 273: 34745-34752.

Carroll, J.C., Pike, C.J. (2008). Selective estrogen receptor modulators differentially regulate Alzheimer-like changes in female 3xTg-AD mice. Endocrinology. 149:2607-11.

Carroll, J.C., Rosario, E., Villamagna, A., Gentzchein, E., Stanczyk, F., Pike, C. (2010). Sex differences in $\beta$-amyloid accumulation in 3xTg-AD mice: role of neonatal sex steroid hormone exposure. Brain Res. 1366, 233. doi:10.1016/j.brainres.2010.10.009 pmid:20934413

Carroll, C. M., and Li, Y. M. (2016). Physiological and pathological roles of the $\mathrm{Y}$-secretase complex. Brain Res. Bull. 126, 199-206. doi: 10.1016/j.brainresbull.2016.04.019

Castellano, J.M., Kim, J., Stewart, F.R., Jiang, H., DeMattos, R.B., Patterson, B.W., et al. (2011). Human apoE isoforms differentially regulate brain amyloid- $\beta$ peptide clearance. Sci Transl Med 3: 89ra57.

Chapman, R.M., Mapstone, M., Gardner, M.N., Sandoval, T.C., McCrary, J.W., Guillily, M.D., Reilly, L.A., Degrush, E. (2011). Women have Farther to Fall: Gender Differences Between Normal 
Elderly and Alzheimer's Disease in Verbal Memory Engender Better Detection of Alzheimer's Disease in Women. J Int Neuropsychol Soc. 1-9.

Charlton, B.M., Rich-Edwards, J.W., Colditz, G.A., et al. (2014). Oral contraceptive use and mortality after 36 years of follow-up in the Nurses' Health Study: prospective cohort study. BMJ. 349:g6356

Chaturvedi, M. and Kaczmarek, L. (2014). Mmp-9 inhibition: a therapeutic strategy in ischemic stroke. Mol. Neurobiol. 49, 563- 573

Chen, J., Chopp, M., Li, Y. (1999). Neuroprotective effects of progesterone after transient middle cerebral artery occlusion in rat. J Neurol Sci. 171:24-30. [PubMed: 10567046]

Chen, M. (2015). The maze of APP processing in Alzheimer's disease: where did we go wrong in reasoning? Front. Cell. Neurosci. 9:186. doi: 10.3389/fncel.2015.00186

Cheng, I. H., Scearce-Levie, K., Legleiter, J., Palop, J., Gerstein, H., et al. (2007). Accelerating amyloid-beta fibrillization reduces oligomer levels and functional deficits in Alzheimer disease mouse models. The Journal of biological chemistry. 282:23818-23828.

Colciaghi, F., Borroni, B., Pastorino, L., Marcello, E., Zimmermann, M., Cattabeni, F., Padovani, A., Luca, M.D. (2002). [alpha]-Secretase ADAM10 as well as [alpha]APPs is reduced in platelets and CSF of Alzheimer disease patients. Mol. Med. 8, 67-74.

Conant, K., Wang, Y., Szklarczyk, A., Dudak, A., Mattson, M. P., Lim S. T. (2010) Matrix metalloproteinase-dependent shedding of intercellular adhesion molecule-5 occurs with long-term potentiation. Neuroscience $166,508-521$. 
Cooke, B.M., Woolley, C.S. (2005) Gonadal hormone modulation of dendrites in the mammalian CNS. J Neurobiol 64:34-46

Copin, J. C., Goodyear, M. C., Gidday, J. M., Shah, A. R., Gascon, E., Dayer, A., et al. (2005). Role of matrix metalloproteinases in apoptosis after transient focal cerebral ischemia in rats and mice. Eur. J. Neurosci. 22, 1597-1608. doi:10.1111/j.1460-9568.2005.04367.x

Corder, E.H., Saunders, A.M., Strittmatter, W.J., Schmechel, D.E., Gaskell, P.C., Small, G.W., et al. (1993). Gene dose of apolipoprotein E type 4 allele and the risk of Alzheimer's disease in late onset families. Science 261: 921-923.

Costa, S., Planchenault, T., Charriere-Bertrand, C., Mouchel ,Y., Fages, C., Juliano, S., Lefrancois, T., Barlovatz-Meimon, G., Tardy, M. (2002). Astroglial permissivity for neuritic outgrowth in neuron-astrocyte cocultures depends on regulation of laminin bioavailability. Glia 37: 105113.

Craft, S. (2009). The role of metabolic disorders in Alzheimer disease and vascular dementia: two roads converged. Arch Neurol, 66:300-305.

Craig, M.C., Murphy, D.G. (2010). Estrogen therapy and Alzheimer's dementia. Ann. N. Y. Acad. Sci., $1205: 245-253$

Cruz-Topete D, and Cidlowski JA. (2015) One Hormone, Two Actions: Anti- and Pro-Inflammatory Effects of Glucocorticoids. Neuroimmunomodulation. 22:20-32 DOI: 10.1159/000362724

De Bosscher, K., Vanden Berghe, W., and Haegeman, G. (2003). The interplay between the glucocorticoid receptor and nuclear factor-kB or activator protein-1: molecular mechanisms for gene repression. Endocr. Rev. 24, 488-522. doi: 10.1210/er.2002-0006 
De Geyter, C., De Geyter, M., Huber, P.R., Nieschlag, E., Holzgreve, W. (2002). Progesterone serum levels during the follicular phase of the menstrual cycle originate from crosstalk between the ovaries and the adrenal cortex. Hum. Reprod. 17:933-39

de Kloet, E.R., Oitzl, M.S., Joëls, M. (1999). Stress and cognition: Are corticosteroids good or bad guys? Trends Neurosci 22: 422-426

de Paiva, C. S., Corrales, R. M., Villarreal, A. L., Farley, W. J., Li, D.-Q., Stern, M. E., et al. (2006). Corticosteroid and doxycycline suppress MMP-9 and inflammatory cytokine expression, MAPK activation in the corneal epithelium in experimental dry eye. Exp. Eye Res. 83, 526535. doi: 10.1016/j.exer.2006.02.004

De Silva, T.M., Faraci, F.M. (2016). Microvascular dysfunction and cognitive impairment. Cell. Mol. Neurobiol. 36:241-258.

De Strooper, B. (2010). Proteases and proteolysis in Alzheimer disease: a multifactorial view on the disease process. Physiol. Rev. 90, 465-494. doi: 10.1152/physrev.00023.2009

Deb S. and Gottschall P. E. (1996). Increased production of matrix metalloproteinases in enriched astrocyte and mixed hippocampal cultures treated with beta-amyloid peptides. J. Neurochem. 66, 1641-1647.

Deb, S., and Gottschall, P. E. (1996). Increased production of matrix metalloproteinases in enriched astrocyte and mixed hippocampal cultures treated with $\beta$-amyloid peptides. J. Neurochem. 66, 1641-1647. doi: 10.1046/j.1471-4159.1996.66041641.x

Deb, S., Wenjun Zhang, J., and Gottschall, P. E. (2003). $\beta$-amyloid induces the production of active, matrix-degrading proteases in cultured rat astrocytes. Brain Res. 970, 205-213. doi: 10.1016/s0006-8993(03)02344-8 
Dejonckheere E., Vandenbroucke R. E., Libert C. (2011). Matrix metalloproteinases as drug targets in ischemia/reperfusion injury. Drug Discov. Today 16, 762-778.

doi:10.1016/j.drudis.2011.06.009

Del Rio, J.P., Alliende, M.I., Molina, N., Serrano, F.G., Molina, S., Vigil, P. (2018). Steroid hormones and their action in women's brains: The importance of hormonal balance. Front. Public Health. 6: 141.

Delay, C., Calon, F., Mathews, P., Hébert, S.S. (2011). Alzheimer-specific variants in the 3'UTR of Amyloid precursor protein affect microRNA function. Mol Neurodegener 6: 70.

DeMayo, F.J., Zhao, B., Takamoto, N., Tsai, SY. (2002). Mechanisms of action of estrogen and progesterone. Annals of the New York Academy of Sciences, 955:48-59 (Discussion 86-88, 396-406)

Desdouits-Magnen, J., Desdouits, F., Takeda, S., Syu, L.J., Saltiel, A.R., Buxbaum, J.D., Czernik, A.J., Nairn, A.C., Greengard, P. (1998). Regulation of secretion of Alzheimer amyloid precursor protein by the mitogen activated protein kinase cascade. J Neurochem 70:524-530.

Desmet, S.J., De Bosscher, K. (2017). Glucocorticoid receptors: finding the middle ground. J Clin Invest. 127:1136-45. doi:10.1172/JCl88886

Di Nezza, L. A., Jobling, T., Salamonsen, L. A. (2003). Progestin suppresses matrix metalloproteinase production in endometrial cancer. Gynecol. Oncol. 89, 325-333. doi: 10.1016/s00908258(03)00089-1

Diotel N., Charlier TD., d’Hellencourt, CL., Couret D., Trudeau VL., Nicolau JC., et al. (2018). Steroid Transport, Local Synthesis, and Signaling within the Brain: Roles in Neurogenesis, Neuroprotection, and Sexual Behaviors. Front. Neurosci. 12:84. doi:

10.3389/fnins.2018.00084 
Dityatev A., Schachner M., Sonderegger P. (2010). The dual role of the extracellular matrix in synaptic plasticity and homeostasis. Nature Reviews Neuroscience. 11(11):735-746. doi: 10.1038/nrn2898.

Dotson, V.M., Beydoun, M.A., Zonderman, A.B. (2010). Recurrent depressive symptoms and the incidence of dementia and mild cognitive impairment. Neurology 75, 27-34.

Dziembowska M. and Wlodarczyk J. (2012) MMP9: a novel function in synaptic plasticity. Int. J. Biochem. Cell Biol. 44, 709-713.

Dzwonek, J., Rylski, M., and Kaczmarek, L. (2004). Matrix metalloproteinases and their endogenous inhibitors in neuronal physiology of the adult brain. FEBS Lett. 567, 129-135. doi: 10.1016/j.febslet.2004.03.070

Eberhardt, W., Schulze, M., Engels, C., Klasmeier, E., and Pfeilschifter, J. (2002). Glucocorticoidmediated suppression of cytokine-induced matrix metalloproteinase- 9 expression in rat mesangial cells: involvement of nuclear factor-kB and Ets transcription factors. Mol. Endocrinol. 16, 1752-1766. doi: 10.1210/me.2001-0278

Epperson, C.N., Sammel, M.D., Freeman, E.W. (2013) Menopause effects on verbal memory: findings from a longitudinal community cohort. J Clin Endocrinol Metab. 98:3829-38.

Espeland, M.A, Shumaker, S.A., Leng, I., Manson, J.E., Brown, C.M., LeBlanc, E.S., et al. (2013). Longterm effects on cognitive function of postmenopausal hormone therapy prescribed to women aged 50 to 55 years. JAMA Intern Med. 173:1429-36

Ethell, IM., and Ethell, DW. (2007). Matrix metalloproteinases in brain development and remodeling: synaptic functions and targets. J. Neurosci. Res. 85, 2813-2823. doi: 10.1002/jnr.2127 
Farrer, L.A., Cupples, L.A., Haines, J.L. et al. (1997). Effects of age, sex, and ethnicity on the association between apolipoprotein E genotype and Alzheimer disease. A meta-analysis. APOE and Alzheimer Disease Meta Analysis Consortium. JAMA 278, 1349-1356.

Fellgiebel, A., Kojro, E., Müller, M.J., Scheurich, A., Schmidt, L.G., Fahrenholz, F. (2009). CSF APPs alpha and phosphorylated tau protein levels in mild cognitive impairment and dementia of Alzheimer's type. J. Geriatr. Psychiatry Neurol. 22, 3-9.

Ferguson, BR., and Gao, WJ. (2018). Thalamic control of cognition and social behavior via regulation of $\gamma$-aminobutyric acidergic signaling and excitation/inhibition balance in the medial prefrontal cortex. Biol. Psychiatry 83, 657-669. doi: 10.1016/j.biopsych.2017.11.033

Fields, RD., Araque, A., Johansen-Berg, H., Lim, SS., Lynch, G., Nave, KA., et al. (2014). Glial biology in learning and cognition. Neuroscientist 20, 426-431. doi: 10.1177/1073858413504465

Filippov, AV., Suleimanova, AV., Grobner, G., and Antsutkin, ON. (2008). Effect of freezing on amyloid peptide aggregation and self-diffusion in an aqueous solution. Colloid J. 70, 501506. doi: $10.1134 / s 1061933 \times 08040157$

Filippov, MA., and Dityatev, A. (2012). Matrix metalloproteinase-9 and non-amyloidogenic pathway of amyloid precursor protein processing. J. Neurochem. 121, 181-183. doi: 10.1111/j.14714159.2011.07641.x

Filon, JR., Intorcia, AJ., Sue, LI., Vazquez Arreola, E., Wilson, J., Davis, KJ., et al. (2016). Gender differences in Alzheimer disease: brain atrophy, histopathology burden, and cognition. J Neuropathol Exp Neurol. 75:748-54

Fisher, DW., Bennett, DA., and Dong, H. (2018). Sexual dimorphism in predisposition to Alzheimer's disease. Neurobiol. Aging 70, 308-324. doi:10.1016/j.neurobiolaging.2018.04.004 
Fleisher AS, Sun S, Taylor C et al. (2008). Volumetric MRI vs clinical predictors of Alzheimer disease in mild cognitive impairment. Neurology 70, 191-199.

Follesa P, Porcu P, Sogliano C, Cinus M, Biggio F, Mancuso L, Mostallino MC, Paoletti AM, Purdy RH, Biggio $G$, Concas A (2002). Changes in GABAA receptor 2 subunit gene expression induced by long-term administration of oral contraceptives in rats. Neuropharmacology 42:325-336.

Fossati S., Giannoni P., Solesio M.E., Cocklin S.L., Cabrera E., Ghiso J., Rostagno A. (2016). The carbonic anhydrase inhibitor methazolamide prevents amyloid beta-induced mitochondrial dysfunction and caspase activation protecting neuronal and glial cells in vitro and in the mouse brain. Neurobiol. Dis. 86:29-40.

Fragkouli, A., Tsilibary, E. C., and Tzinia, A. K. (2014). Neuroprotective role of MMP-9 overexpression in the brain of Alzheimer's 5xFAD mice. Neurobiol. Dis. 70, 179-189. doi: 10.1016/j.nbd.2014.06.021

Frankowski, H., Gu, Y. H., Heo, J. H., Milner, R., and Del Zoppo, G. J. (2012). Use of gel zymography to examine matrix metalloproteinase (Gelatinase) expression in brain tissue or in primary glial cultures. Biology 814, 221-233. doi: 10.1007/978-1-61779-452-0_15

Gabriel, A., and Fahim, G. (2005). Do depot medroxyprogesterone acetate contraceptive injections cause mood changes and memory impairment? Prim Psychiatry 12, 59-60.

Galvez-Peralta. "Estrogens and Progestins." West Virginia University, Morgantown, November 2015. Lecture.

Gambrell RD Jr. (1986). The role of hormones in the etiology and prevention of endometrial cancer. Clin Obstet Gynaecol. 13:695-723. [PubMed: 3791826] 
Ganguly K., Rejmak E., Mikosz M., Nikolaev E., Knapska E. and Kaczmarek L. (2013) Matrix metalloproteinase (MMP) 9 transcription in mouse brain induced by fear learning. J. Biol. Chem. 288, 20978-20991.

Gargano J, Reeves M. (2007). Sex differences in stroke recovery and stroke-specific quality of life. Stroke. 38:2541-2548

Gawlak M, Górkiewicz T, Gorlewicz A, Konopacki FA, Kaczmarek L, Wilczynski GM (2009). High resolution in situ zymography reveals matrix metalloproteinase activity at glutamatergic synapses. Neuroscience 158(1):167-176

Gawlak, M., Górkiewicz, T., Gorlewicz, A., Konopacki, F. A., Kaczmarek, L., Wilczynski, G. M. (2009). High resolution in situ zymography reveals matrix metalloproteinase activity at glutamatergic synapses. Neuroscience 158, 167-176. doi:10.1016/j.neuroscience.2008.05.045

Genersch, E., Hayess, K., Neuenfeld, Y., and Haller, H. (2000). Sustained ERK phosphorylation is necessary but not sufficient for MMP-9 regulation in endothelial cells: involvement of Rasdependent and-independent pathways. J. Cell Sci. 113, 4319-4330.

George SJ, Johnson JL (2010). In situ zymography. In: Matrix Metalloproteinase Protocols. Springer, pp 271-277

Ghiso, J., and Frangione, B. (2002). Amyloidosis and Alzheimer's disease. Adv. Drug Deliv. Rev. 54, 1539-1551. doi: 10.1016/s0169-409x(02)00149-7

Giatti, S., Melcangi, R. C., and Pesaresi, M. (2016). The other side of progestins: effects in the brain. J. Mol. Endocrinol. 57, R109-R126. doi: 10.1530/jme-16-0061 
Giraudon P., Buart S., Bernard A., Thomasset N. and Belin M. F. (1996). Extracellular matrixremodeling metalloproteinases and infection of the central nervous system with retrovirus human T-lymphotropic virus type I (HTLV-I). Prog. Neurobiol. 49, 169-184.

Gleason CE, Dowling NM, Wharton W, Manson JE, Miller VM, Atwood CS, et al. (2015) Effects of hormone therapy on cognition and mood in recently postmenopausal women: findings from the Randomized, Controlled KEEPS-Cognitive and Affective Study. PLoS

Goedeke L, Fernández-Hernando C. (2014). MicroRNAs: A connection between cholesterol metabolism and neurodegeneration. Neurobiol Dis 72: 48-53.

Gong, Y., Chang, L., Viola, KL., Lacor, PN., Lambert, MP., Finch, CE., Krafft, GA., Klein, WL. (2003). Alzheimer's disease-affected brain: presence of oligomeric A beta ligands (ADDLS) suggests a molecular basis for reversible memory loss. Proceedings of the National Academy of Sciences of the United States of America $100,10417-10422$.

Goodman Y, Bruce AJ, Cheng B, Mattson MP. (1996). Estrogens attenuate and corticosterone exacerbates excitotoxicity, oxidative injury, and amyloid beta-peptide toxicity in hippocampal neurons. J Neurochem. 66:1836-44. [PubMed: 8780008]

Gorkiewicz T., Balcerzyk M., Kaczmarek L. and Knapska E. (2015) Matrix metalloproteinase 9 (MMP9) is indispensable for long term potentiation in the central and basal but not in the lateral nucleus of the amygdala. Front. Cell Neurosci. 9, 73

Gorkiewicz, T., Balcerzyk, M., Kaczmarek, L., and Knapska, E. (2015). Matrix metalloproteinase 9 (MMP-9) is indispensable for long term potentiation in the central and basal but not in the lateral nucleus of the amygdala. Front. Cell. Neurosci. 9:73. doi: 10.3389/fncel.2015.00073 
Götz J, Chen F, van Dorpe J, Nitsch RM. (2001). Formation of neurofibrillary tangles in P301I tau transgenic mice induced by Abeta 42 fibrils. Science 293: 1491-5 (2001)

Gouras GK, Xu H, Gross RS, Greenfield JP, Hai B, Wang R, Greengard P. (2000). Testosterone reduces neuronal secretion of Alzheimer's beta-amyloid peptides. Proc. Natl. Acad. Sci. U. S. A. $97,1202-1205$.

Graham JD, Clarke CL. (1997) Physiological action of progesterone in target tissues. Endocr Rev $18: 502-519$

Greendale GA, Huang MH, Wight RG, Seeman T, Luetters C, Avis NE, et al. (2009). Effects of the menopause transition and hormone use on cognitive performance in midlife women. Neurology. 72:1850-7

Greenfield JP, Leung LW, Cai D, Kaasik K, Gross RS, Rodriguez-Boulan E, Greengard P, Xu H. (2002). Estrogen lowers Alzheimer beta-amyloid generation by stimulating trans-Golgi network vesicle biogenesis. J Biol Chem. 277: 12128-12136. 10.1074/jbc.M110009200.

Griciuc A, Serrano-Pozo A, Parrado AR, Lesinski AN, Asselin CN, Mullin K, et al. (2013). Alzheimer's disease risk gene CD33 inhibits microglial uptake of amyloid beta. Neuron 78: 631-43.

Grimm MO, Mett J, Stahlmann C, Haupenthal V, Zimmer V, Hartmann T. (2013). Neprilysin and A clearance: impact of the APP intracellular domain in NEP regulation and implications in Alzheimer's disease. Front. Aging Neurosci. 5, 98 
Grundke-Iqbal I, Iqbal K, Quinlan M, Tung YC, Zaidi MS, Wisniewski HM. (1986). Microtubuleassociated protein tau. A component of Alzheimer paired helical filaments. J Biol Chem 261: 6084-9.

Gurvich C, Hoy K, Thomas N, Kulkarni J. (2018). Sex Differences and the Influence of Sex Hormones on Cognition through Adulthood and the Aging Process. Brain Sci. 8:163.

Haass, C., and Selkoe, D. J. (2007). Soluble protein oligomers in neurodegeneration: lessions from the Alzheimer's amyloid beta-peptide. Nat. Rev. Mol. Cell Biol. 8, 101-112. doi: $10.1038 / \mathrm{nrm} 2101$

Hackenberg R, Hofmann J, Wolff G, Holzel F, Schulz KD. (1990). Down-regulation of androgen receptor by progestins and interference with estrogenic or androgenic stimulation of mammary carcinoma cell growth. J. Cancer Res. Clin. Oncol., 116:492-498

Hajszan, T., MacLusky, NJ., Leranth, C. (2008). Role of androgens and the androgen receptor in remodeling of spine synapses in limbic brain areas. Horm Behav. 53:638-46. [PubMed: 18262185]

Halford RW, Russell DW (2009). Reduction of cholesterol synthesis in the mouse brain does not affect amyloid formation in Alzheimer's disease, but does extend lifespan. Proc Natl Acad Sci U S A 106:3502-3506.

Hall JR, Vo HT, Johnson LA, Wiechmann A, O'Bryant SE. (2012). Bonston naming test: gender differences in older adults with and without Alzheimer's dementia. Psychology. 3:485-488.

Hanamsagar R, Bilbo SD. (2016). Sex differences in neurodevelopmental and neurodegenerative disorders: focus on microglial function and neuroinflammation during development. J Steroid Biochem Mol Biol. 160:127-133. 
Hardy, J. (2009). The amyloid hypothesis for Alzheimer's disease: a critical reappraisal. J. Neurochem. 110, 1129-1134.

Hatanaka Y., Mukai H., Mitsuhashi K., Hojo,Y.,Murakami G., Komatsuzaki Y., Sato R., Kawato S. (2009) Androgen rapidly increases dendritic thorns of CA3 neurons in male rat hippocampus. Biochem. Biophys. Res. Commun.381,728-732.

Hayashita-Kinoh H, Kinoh H, Okada A, Komori K, Itoh Y, Chiba T, Kajita M, Yana I, Seiki M (2001) Membrane-type 5 matrix metalloproteinase is expressed in differentiated neurons and regulates axonal growth. Cell Growth Differ 12: 573-580.

Haydon, P. G. (2001). Glia: listening and talking to the synapse. Nat. Rev. Neurosci. 2, 185-193. doi: $10.1038 / 35058528$

Hébert SS, De Strooper B. (2009). Iterations of the microRNA network cause neurodegenerative disease. Trends Neurosci 32: 199-206.

Helbecque N., Hermant X., Cottel D. and Amouyel P. (2003) The role of matrix metalloproteinase-9 in dementia. Neurosci. Lett. 350, 181- 183.

Henderson VW, St John JA, Hodis HN, McCleary CA, Stanczyk FZ, Shoupe D, et al. (2016). Cognitive effects of estradiol after menopause: a randomized trial of the timing hypothesis. Neurology 87:699-708

Hernandez-Guillamon M, Delgado P, Ortega L et al., (2009). Neuronal TIMP-1 release accompanies astrocytic MMP-9 secretion and enhances astrocyte proliferation induced by $\beta$-amyloid 2535 fragment," Journal of Neuroscience Research. 87:2115-2125. 
Hernandez-Guillamon, M., Mawhirt, S., Blais, S., Montaner, J., Neubert, T. A., Rostagno, A., et al. (2015). Sequential amyloid- $\beta$ degradation by the matrix metalloproteases MMP-2 and MMP-9. J. Biol. Chem. 290, 15078-15091. doi: 10.1074/jbc.M114.610931

Hickman SE, El Khoury J. (2014). TREM2 and the neuroimmunology of Alzheimer's disease. Biochem Pharmacol 88: 495-8.

Hiller-Sturmhöfel, S., Bartke, A. (1998) The Endocrine System. Alcohol Health Res. World. 22:153154.

Hirata-Fukae C., Li H.F., Hoe H.S., Gray A.J., Minami S.S., Hamada K., Niikura T., Hua F., TsukagoshiNagai H., et al., (2008) Females exhibit more extensive amyloid, but not tau, pathology in an Alzheimer transgenic model. Brain Res., 24:92-103.

Hirvonen E. Progestins. Maturitas. (1996). 23(Suppl):S13-8. [PubMed: 8865133]

Hof PR, Giannakopoulos P, Bouras C. (1996). The neuropathological changes associated with normal brain aging. Histol Histopathol. 11:1075-1088.

Hogervorst, E., Yaffe, K., Richards, M., Huppert, F. (2002). Hormone replacement therapy for cognitive function in postmenopausal women. Cochrane Database Syst. Rev., CD003122.

Holland D, Desikan RS, Dale AM, McEvoy LK. (2013). Alzheimer's Disease Neuroimaging Initiative. Higher rates of decline for women and apolipoprotein E epsilon4 carriers. AJNR Am J Neuroradiol. 34:2287-93

Hollingworth P, Harold D, Sims R, Gerrish A, Lambert J-C, Carrasquillo MM, et al. (2011). Common variants at $A B C A 7, M S 4 A 6 A / M S 4 A 4 E, E P H A 1, C D 33$ and CD2AP are associated with Alzheimer's disease. Nat Genet 43: 429-35. 
Holsinger, R. M. D., McLean, C. A., Beyreuther, K., Masters, C. L., and Evin, G. (2002). Increased expression of the amyloid precursor $\beta$-secretase in Alzheimer's disease. Ann. Neurol. 51, 783-786. doi: 10.1002/ana.10208

Holter MC, Hewitt LT, Nishimura KJ, Bjorklund GR, Shah Shiv et al., (2019). Hyperactive MEK1 signaling in cortical GABAergic neurons causes embryonic parvalbumin-neuron death and defects in behavioral inhibition. bioRxiv. 748087; doi:10.1101/748087

Honig, L.S., Tang, M.X., Albert, S., Costa, R., Luchsinger, J., Manly, J., Stern, Y., and Mayeux, R. (2003) Stroke and the risk of Alzheimer disease. Arch. Neurol., 60:1707-1712.

Horder H, Johansson L, Guo X et al. (2018) Midlife cardiovascular fitness and dementia: A 44-year longitudinal population study in women. Neurology 90, e1298-e1305.

Hormones, Brain and Behavior by Pfaff and Joels, $3^{\text {rd }}$ edition (2016, American Press) Hu, J., Zhang, Z., Shen, W.J., Azhar, S. (2010). Cellular cholesterol delivery, intracellular processing and utilization for biosynthesis of steroid hormones. Nutr Metab (Lond). 7:47.

Hu Q., Chen C., Yan J., Yang X., Shi X., Zhao J., et al. . (2009). Therapeutic application of gene silencing MMP-9 in a middle cerebral artery occlusion-induced focal ischemia rat model. Exp. Neurol. 216, 35-46. 10.1016/j.expneurol.2008.11.007

Hu W, Zhang Y, Wu W, Yin T, Huang D, Wang Y et al., (2016) Chronic glucocorticoids exposure enhances neurodegeneration in the frontal cortex and hippocampus via NLRP-1 inflammasome activation in male mice. Brain, Behavior, and Immunity. 52:58-70.

Hua X, Hibar DP, Lee S, Toga AW, Jack CR Jr,WeinerMW, et al. (2010). Sex and age differences in atrophic rates: an ADNI study with n51368 MRI scans. Neurobiol Aging. 31:1463-80 
Hudson, W. H., Vera, I. M. S. D., Nwachukwu, J. C., Weikum, E. R., Herbst, A. G., Yang, Q., et al. (2018). Cryptic glucocorticoid receptor-binding sites pervade genomic NF-kB response elements. Nat. Commun. 9:1337. doi: 10.1038/s41467-018-03780-1

Huppenbauer CB, Tanzer L, DonCarlos LL, Jones, KJ. (2005) Gonadal steroid attenuation of developing hamster facial motoneuron loss by axotomy: equal efficacy of testosterone, dihydrotestosterone, and 17-beta estradiol. J Neurosci. 25:4004-13. [PubMed: 15843602]

Hussain S, Assender JW, Bond M, Wong LF, Murphy D, Newby AC. (2002) Activation of protein kinase Czeta is essential for cytokine-induced metalloproteinase- $1,-3$, and -9 secretion from rabbit smooth muscle cells and inhibits proliferation. J Biol Chem 277: 27345-27352.

Hwang-Levine, J., Stanczyk, F. Z., and Hodis, H. N. (2011). The role of progestogens in regulating matrix metalloproteinase activity in macrophages and microglial cells. Neurochem. Res. 36, 1870-1875. doi: 10.1007/s11064-011-0508-0

ladecola C (2013) The pathobiology of vascular dementia. Neuron 80(4):844-866. doi:10.1016/j.neuron.2013.10.008

Inhoffen, H. H., and Hohlweg, W., Naturwiss., 26, 96 (1938)

Institute of Medicine (2001). Exploring the Biological Contributions to Human Health: Does Sex Matter?. Washington, DC: The National Academies Press; 10.17226/10028.

Irvine K, Laws KR, Gale TM, Kondel TK. (2012). Greater cognitive deterioration in women than men with Alzheimer's disease: a meta analysis. J Clin Exp Neuropsychol. 34:989-998.

Ising C, Stanley M, Holtzman D. (2015). Current thinking on the mechanistic basis of Alzheimer's and implications for drug development. Clin Pharmacol Ther 98: 469-71. 
Jacobsen B, Horwitz KB. (2012). Progesterone Receptors, their Isoforms and Progesterone Regulated Transcription. Mol Cell Endocrinol. 357:18-29.

Jaffe AB, Toran-Allerand CD, Greengard P, Gandy SE. (1994). Estrogen regulates metabolism of Alzheimer amyloid $\beta$ precursor protein. J Biol Chem. 269:13065-13068.

Jiang N, Chopp M, Stein D, Feit H. (1996). Progesterone is neuroprotective after transient middle cerebral artery occlusion in male rats. Brain Res. 735:101-7. [PubMed: 8905174]

Jiang T, Yu J-T, Hu N, Tan M-S, Zhu X-C, Tan L. (2013).CD33 in Alzheimer's Disease. Mol Neurobiol 49: 529-535.

Jin R., Yang G. and Li G. (2010). Molecular insights and therapeutic targets for blood-brain barrier disruption in ischemic stroke: critical role of matrix metalloproteinases and tissue-type plasminogen activator. Neurobiol. Dis. 38, 376-385.

Jodhka PK, Kaur P, Underwood W, Lydon JP, Singh M. (2009). The differences in neuroprotective efficacy of progesterone and medroxyprogesterone acetate correlate with their effects on brain-derived neurotrophic factor expression. Endocrinology. 150:3162-3168

Joëls M., Karst H., Krugers H.J., Lucassen P.J. (2007). Chronic stress: implications for neuronal morphology, function and neurogenesis. Front. Neuroendocrinol. 28:72-96

Jonat, C., Rahmsdorf, H. J., Park, K. K., Cato, A. C. B., Gebel, S., Ponta, H., et al. (1990). Antitumor promotion and antiinflammation: down-modulation of AP-1 (Fos/Jun) activity by glucocorticoid hormone. Cell 62, 1189-1204. doi: 10.1016/0092-8674(90)90395-u

Jović, M.; Lončarević-Vasiljković, N.; Ivković, S.; Dinić, J.; Milanović, D.; Zlokovic, B.; Kanazir, S. (2019). Short-term fish oil supplementation applied in presymptomatic stage of Alzheimer's 
disease enhances microglial/macrophage barrier and prevents neuritic dystrophy in parietal cortex of 5xFAD mouse model. PLOS ONE. 14:e0216726.

Kaczmarek, L. (2016). MMP-9 in control of synaptic plasticity: a subjective account. OMandP 2, 103-111.

Kamat, P. K., Swarnkar, S., Rai, S., Kumar, V., and Tyagi, N. (2014). Astrocyte mediated MMP-9 activation in the synapse dysfunction: an implication in Alzheimer disease. Ther. Targets Neurol. Dis. 1:e243. doi: 10.14800/ttnd.243

Kaminari A, Giannakas N, Tzinia A, Tsilibary EC. (2017). Overexpression of matrix metalloproteinase9 (MMP-9) rescues insulin-mediated impairment in the 5XFAD model of Alzheimer's disease. Sci Rep 7:683. doi:10.1038/s41598-017-00794-5

Kaur P, Jodhka PK, Underwood WA, Bowles CA, de Fiebre NC, de Fiebre CM, Singh M. (2007).

Progesterone increases brain-derived neuroptrophic factor expression and protects against glutamate toxicity in a mitogen-activated protein kinase- and phosphoinositide-3 kinasedependent manner in cerebral cortical explants. J Neurosci Res. 85:2441-9. [PubMed: 17549730]

Kauwe JSK, Cruchaga C, Mayo K, Fenoglio C, Bertelsen S, Nowotny P, et al. (2008). Variation in MAPT is associated with cerebrospinal fluid tau levels in the presence of amyloid-beta deposition. Proc Natl Acad Sci USA 105: 8050-4.

Kawas C, Resnick S, Morrison A, Brookmeyer R, Corrada M, Zonderman A, Bacal C, Lingle DD, Metter E. (1997). A prospective study of estrogen replacement therapy and the risk of developing Alzheimer's disease: the Baltimore Longitudinal Study of Aging. Neurology. 48:1517-1521. 
Kesner, RP., Hunt, ME., Williams, JM., and Long, JM. (1996). Prefrontal cortex and working memory for spatial response, spatial location, and visual object information in the rat. Cereb. Cortex 6, 311-318. doi: 10.1093/cercor/6.2.311

Kessler RC, McGonagle KA, Swartz M et al. (1993) Sex and depression in the National Comorbidity Survey. I: Lifetime prevalence, chronicity and recurrence. J Affect Disord 29, 85-96.

Kiko T, Nakagawa K, Tsuduki T, Furukawa K, Arai H, Miyazawa T. (2014). MicroRNAs in plasma and cerebrospinal fluid as potential markers for Alzheimer's disease. J Alzheimers Dis 39: 253-59

Kim J, Basak JM, Holtzman DM. (2009). The role of apolipoprotein E in Alzheimer's disease. Neuron 63: 287-303.

Kirton, KT., and Cornette JC. (1974) Return of ovulatory cyclicity following an intramuscular injection of medroxyprogesterone acetate (Provera). Contraception 10: 39-45. 27.

Koldamova R, Staufenbiel M, Lefterov I. (2005). Lack of ABCA1 considerably decreases brain ApoE level and increases amyloid deposition in APP23 mice. J Biol Chem 280: 43224-35.

Koran MEI, Wagener M, Hohman TJ. (2017). Alzheimer's Neuroimaging Initiative. Sex differences in the association between AD biomarkers and cognitive decline. Brain Imaging Behav. 11:205-13.

Koubovec, D., Ronacher, K., Stubsrud, E., Louw, A., and Hapgood, J. P. (2005). Synthetic progestins used in HRT have different glucocorticoid agonist properties. Mol. Cell. Endocrinol. 242, 2332. doi: 10.1016/j.mce.2005.07.001 
Koubovec, D., Vanden Berghe, W., Vermeulen, L., Haegeman, G., and Hapgood, J. P. (2004).

Medroxyprogesterone acetate downregulates cytokine gene expression in mouse fibroblast cells. Mol. Cell. Endocrinol. 221, 75-85. doi: 10.1016/j.mce.2004.03.006

Koyama, Y., Ukita, A., Abe, K., Iwamae, K., Tokuyama, S., Tanaka, K., et al. (2017). Dexamethasone downregulates endothelin receptors and reduces endothelin-induced production of matrix metalloproteinases in culture rat astrocytes. Mol. Pharmacol. 92, 57-66. doi: 10.1124/mol.116.107300

Kumar, S., Holmes, E., Scully, S., Birren, B. W., Wilson, R. H., and de Vellis, J. (1986). The hormonal regulation of gene expression of glial markers: Glutamine synthetase and glycerol phosphate dehydrogenase in primary cultures of rat brain and in C6 cell line. J. Neurosci. Res. 16, 251-264. doi: 10.1002/jnr.490160122

Kumon Y, Kim SC, Tompkins P, Stevens A, Sakaki S, Loftus CM. (2000) Neuroprotective effect of postischemic administration of progesterone in spontaneously hypertensive rats with focal cerebral ischemia. J Neurosurg. 92:848-52. [PubMed: 10794300]

Kuo J, Micevych P. (2013). Neurosteroid, trigger of the LH surge.J Steroid Biochem Mol Biol. 131:5765.

Kwok J. C. F., Dick G., Wang D., Fawcett J. W. (2011) Extracellular matrix and perineuronal nets in CNS repair. Developmental Neurobiology. 71(11):1073-1089. doi: 10.1002/dneu.20974.

Lannfelt L et al., (1995) Decreased alpha-secretase-cleaved amyloid precursor protein as a diagnostic marker for Alzheimer's disease. Nat Med. 1, 829-832.

Leidinger P, Backes C, Deutscher S, Schmitt K, Mueller SC, Frese K, et al. (2013) A blood based 12miRNA signature of Alzheimer disease patients. Genome Biol 14: R78. 
Lepeta, K., and Kaczmarek, L. (2015). Matrix metalloproteinase-9 as a novel player in synaptic plasticity and schizophrenia. Schizophr. Bull. 41, 1003-1009. doi: 10.1093/schbul/sbv036

Leroy K, Ando K, Laporte V, Dedecker R, Suain V, Authelet M, et al. (2012) Lack of tau proteins rescues neuronal cell death and decreases amyloidogenic processing of APP in APP/PS1 mice. Am J Pathol 181: 1928-40.

Lesné, S. E., Kotilinek, L., and Ashe, K. H. (2008). Plaque-bearing mice with reduced levels of oligomeric amyloid- $\beta$ assemblies have intact memory function. Neuroscience 151, 745-749. doi: 10.1016/j.neuroscience.2007.10.054

Lesné, S. E., Sherman, M. A., Grant, M., Kuskowski, M., Schneider, J. A., Bennett, D. A., et al. (2013). Brain amyloid- $\beta$ oligomers in ageing and Alzheimer's disease. Brain 136, 1383-1398. doi: 10.1093/brain/awt062

Levin-Allerhand JA, Lominska CE, Smith JD. (2002) Increased amyloid-levels in APPSWE transgenic mice treated chronically with a physiological high-fat high-cholesterol diet. J Nutr Health Aging. 6:315-319.

Li D. D., Song J. N., Huang H., Guo X. Y., An J. Y., Zhang M., et al. . (2013). The roles of MMP-9/TIMP1 in cerebral edema following experimental acute cerebral infarction in rats. Neurosci. Lett. 550, 168-172. doi:10.1016/j.neulet.2013.06.034

Li, R., and Singh, M. (2014). Sex differences in cognitive impairment and Alzheimer's disease. Front. Neuroendocrinol. 35, 385-403. doi: 10.1016/j.yfrne.2014.01.002

Li, Y.-F., Xu, X.-B., Chen, X.-H., Wei, G., He, B., and Wang, J.-D. (2012). The nuclear factor-kB pathway is involved in matrix metalloproteinase-9 expression in RU486-induced endometrium breakdown in mice. Hum. Reprod. 27, 2096-2106. doi: 10.1093/humrep/des110 
Liang, K., Yang, L., Yin, C., Xiao, Z., Zhang, J., Liu, Y., et al. (2010). Estrogen stimulates degradation of beta amyloid peptide by up-regulating neprilysin. J. Biol. Chem. 285, 935-942. doi: 10.1074/jbc.m109.051664

Lin KA, Choudhury KR, Rathakrishnan BG, Marks DM, Petrella JR, Doraiswamy PM, et al. (2015) Marked gender differences in progression of mild cognitive impairment over 8 years. Alzheimers Dement. 1:103-10 Med 2015;12:e1001833; discussion e1001833

Littleton-Kearney MT, Klaus JA, Hurn PD. (2005) Effects of combined oral conjugated estrogens and medroxyprogesterone acetate on brain infarction size after experimental stroke in rat. J Cereb Blood Flow Metab. 25:421-6. [PubMed: 15689957]

Liu, N. K., and Xu, X. M. (2012). Neuroprotection and its molecular mechanism following spinal cord injury. Neural Regen. Res. 7, 2051-2062. doi: 10.3969/j.issn.1673-5374.2012.26.007

Livingston G, Sommerlad A, Orgeta V et al. (2017) Dementia prevention, intervention, and care. Lancet.

Llano E, Pendas AM, Freije JP, Nakano A, Knauper V, Murphy G, Lopez-Otin C (1999) Identification and characterization of human MT5-MMP, a new membrane-bound activator of progelatinase a overexpressed in brain tumors. Cancer Res 59: 2570-2576.

Long JM, Lahiri DK. (2011) MicroRNA-101 downregulates Alzheimer's amyloid- $\beta$ precursor protein levels in human cell cultures and is differentially expressed. Biochem Biophys Res Commun 404: 889- 95. 
Long JM, Ray B, Lahiri DK. (2012) MicroRNA-153 physiologically inhibits expression of amyloid- $\beta$ precursor protein in cultured human fetal brain cells and is dysregulated in a subset of Alzheimer disease patients. J Biol Chem 287: 31298-310.

Lorenzl S., Albers D. S., Relkin N., Ngyuen T., Hilgenberg S. L., Chirichigno J., Cudkowicz M. E. and Beal M. F. (2003) Increased plasma levels of matrix metalloproteinase-9 in patients with Alzheimer's disease. Neurochem. Int. 43, 191-196

Louw-du Toit, R., Hapgood, J. P., and Africander, D. (2014). Medroxyprogesterone acetate differentially regulates interleukin (IL)-12 and IL-10 in a human ectocervical epithelial cell line in a glucocorticoid receptor (GR)-dependent manner. J. Biol. Chem. 289, 31136-31149. doi: $10.1074 / j b c . m 114.587311$

Lowry, N. C., Pardon, L. P., Yates, M. A., and Juraska, J. M. (2010). Effects of long-term treatment with $17 \beta$-estradiol and medroxyprogesterone acetate on water maze performance in middle aged female rats. Horm. Behav. 58, 200-207. doi: 10.1016/j.yhbeh.2010.03.018

Lublin, A. L., and Gandy, S. (2010). Amyloid- $\beta$ oligomers: possible roles as key neurotoxins in Alzheimer's disease. Mt. Sinai J. Med. 77, 43-49. doi: 10.1002/msj.20160

Lucivero V., Prontera M., Mezzapesa D. M., Petruzzellis M., Sancilio M., Tinelli A., et al. . (2007). Different roles of matrix metalloproteinases- 2 and -9 after human ischaemic stroke. Neurol. Sci. 28, 165-170. doi:10.1007/s10072-007-0814-0

M. C. Velarde MC. (2014). Mitochondrial and sex steroid hormone crosstalk during aging," Longevity \& Healthspan, vol. 3, no. 1, article 2.

M. Schumacher, R. Hussain, N. Gago, J.P. Oudinet, C. Mattern, A.M. Ghoumari. (2012) Progesterone synthesis in the nervous system: implications for myelination and myelin repair. Front Neurosci. 6:10. doi: 10.3389/fnins.2012.00010 
MacLusky NJ, et al. (2004) Effects of dehydroepiandrosterone and flutamide on hippocampal CA1 spine synapse density in male and female rats: implications for the role of androgens in maintenance of hippocampal structure. Endocrinology. 145:4154-61. [PubMed: 15178643]

MacNamara P, Loughrey HC. (1998) Progesterone receptor A and B isoform expression in human osteoblasts. Calcif Tissue Int. 63:39-46

Maki PM. (2005) A systematic review of clinical trials of hormone therapy on cognitive function: effects of age at initiation and progestin use. Ann NY Acad Sci. 1052:182-197

Manson JE, Aragaki AK, Rossouw JE, Anderson GL, Prentice RL, LaCroix AZ, et al. (2017) Menopausal hormone therapy and long-term all cause and cause-specific mortality: The Women's Health Initiative Randomized Trials. JAMA. 318:927-38

Marron TU, Guerini V, Rusmini DS, Brevini TAL, Martini L, Poletti A. (2005) Androgen-induced neurite outgrowth is mediated by neuritin in motor neurones. J Neurochem. 92:10-20. [PubMed: 15606892]

Martínez-Sánchez P, Fuentes B, Fernández-Domínguez J, et al. (2011a) Young Women Have Poorer Outcomes than Men after Stroke. Cerebrovasc Dis. 31:455-463

Martínez-Sánchez P, Fuentes B, Alonso de, Masjuan J, Simal P, Egido J, et al. (2011b) Female gender is a factor of worse outcome in acute stroke even after thrombolytic treatment. Int J Stroke. 6:371-372.

McAllister C, Long J, Bowers A, Walker A, Cao P, Honda S, Harada N, Staufenbiel M, Shen Y, Li R. (2010). Genetic targeting aromatase in male amyloid precursor protein transgenic mice 
down-regulates beta-secretase (BACE1) and prevents Alzheimer-like pathology and cognitive impairment. J Neurosci. 30:7326-34. [PubMed: 20505099]

McEwan, I. J., Wright, A. P. H., and Gustafsson, J. A. (1997). Mechanism of gene expression by the glucocorticoid receptor: role of protein-protein interactions. BioEssays 19, 153-160. doi: 10.1002/bies.950190210

Meda L Cassatella MA Szendrei GI Otvos L Jr Baron P Villalba M, et al. (1995) Activation of microglial cells by beta-amyloid protein and interferon-gamma . Nature. 374 : $647-50$.

Meighan S. E., Meighan P. C., Choudhury P., Davis C. J., Olson M. L., Zornes P. A., Wright J. W. and Harding J. W. (2006) Effects of extracellular matrix-degrading proteases matrix metalloproteinases 3 and 9 on spatial learning and synaptic plasticity. J. Neurochem. 96, $1227-1241$.

Meijsing, S. H., Pufall, M. A., So, A. Y., Bates, D. L., Chen, L., and Yamamoto, K. R. (2009). DNA binding site sequence directs glucocorticoid receptor structure and activity. Science 324, 407-410. doi: 10.1126/science.1164265

Merlo, S., and Sortino, M. A. (2012). Estrogen activates matrix metalloproteinases-2 and -9 to increase $\beta$ amyloid degradation. Mol. Cell. Neurosci. 49, 423-429. doi: 10.1016/j.mcn.2012.02.005

Micevych P, Soma KK, Sinchak K. (2008) Neuroprogesterone: key to estrogen positive feedback? Brain Res Rev 57:470-480.

Michaluk P., Wawrzyniak M., Alot P. et al. (2011) Influence of matrix metalloproteinase MMP-9 on dendritic spine morphology. J. Cell Sci. 124, 3369-3380.

Michaluk, P., Wawrzyniak, M., Alot, P., Szczot, M., Wyrembek, P., Mercik, K., et al. (2011). Influence of matrix metalloproteinase MMP-9 on dendritic spine morphology. J. Cell Sci. 124, 33693380. doi: $10.1242 / j c s .090852$ 
Mielke MM, Vemuri P, Rocca WA (2014) Clinical epidemiology of Alzheimer's disease: assessing sex and gender differences. Clin Epidemiol; 6: 37-48

Mittelstadt, M. L., and Patel, R. C. (2012). AP-1 mediated transcriptional repression of matrix metalloproteinase- 9 by recruitment of histone deacetylase 1 in response to interferon B. PLoS One 7:e42152. doi: 10.1371/journal.pone.0042152

Miyagawa K, Vidgoff J, Hermsmeyer K. (1997) Ca2+ release mechanism of primate drug-induced coronary vasospasm. Am J Physiol. 272:H2645-54. [PubMed: 9227542]

Mizoguchi, H., Takuma, K., Fukuzaki, E. et al., (2009) Matrix metalloprotease-9 inhibition improves amyloid $\beta$-mediated cognitive impairment and neurotoxicity in mice. Journal of Pharmacology and Experimental Therapeutics. 331:14-22.

Mizoguchi, H., Takuma, K., Fukuzaki, E., Ibi, D., Someya, E., Akazawa, K.-H., et al. (2009). Matrix metalloprotease-9 inhibition improves amyloid-mediated cognitive impairment and neurotoxicity in mice. J. Pharmacol. Exp. Ther. 331, 14-22. doi: 10.1124/jpet.109.154724

Moffat SD, Zonderman AB, Metter EJ, Kawas C, Blackman MR, Harman SM, Resnick SM. (2004) Free testosterone and risk for Alzheimer disease in older men. Neurology. 62:188-93

Moldes O., Sobrino T., Millán M., Castellanos M., Pérez de la Ossa N., Leira R., et al. (2008). High serum levels of endothelin-1 predict severe cerebral edema in patients with acute ischemic stroke treated with t-PA. Stroke 39, 2006-2010. doi:10.1161/STROKEAHA.107.495044

Montaner J., Alvarez-Sabín J., Molina C., Anglés A., Abilleira S., Arenillas J., et al.(2001). Matrix metalloproteinase expression after human cardioembolic stroke: temporal profile and relation to neurological impairment. Stroke 32, 1759-1766. doi:10.1161/01.STR.32.8.1759 
Morali G, Letechipia-Vallejo G, Lopez-Loeza E, Montes P, Hernandez-Morales L, Cervantes M. (2005) Post-ischemic administration of progesterone in rats exerts neuroprotective effects on the hippocampus. Neurosci Lett.382:286-90. [PubMed: 15885907]

Mosher WD, Jones J. Use of contraception in the United States: 1982-2008. Vital Health Stat 232010;(29):1-44.

Mroczko B., Groblewska M., Zboch M., Kulczynska A., Koper O. M., Szmitkowski M., Kornhuber J. and Lewczuk P. (2014) Concentrations of Matrix Metalloproteinases and their Tissue Inhibitors in the Cerebrospinal Fluid of Patients with Alzheimer's Disease. J. Alzheimers Dis. 40, 351-357.

Mucke, L., Masliah, E., Yu, GQ., Mallory, M., Rockenstein, EM., Tatsuno, G., Hu, K., Kholodenko, D., Johnson-Wood, K., McConlogue, L. (2000). High-level neuronal expression of abeta 1-42 in wild-type human amyloid protein precursor transgenic mice: synaptotoxicity without plaque formation. The Journal of neuroscience: the official journal of the Society for Neuroscience 20 , 4050-4058.

Murakami K, Irie K, Morimoto A, Ohigashi H, Shindo M, Nagao M, et al. (2003) Neurotoxicity and physicochemical properties of Abeta mutant peptides from cerebral amyloid angiopathy: Implication for the pathogenesis of cerebral amyloid angiopathy and Alzheimer's disease. J Biol Chem 278: 46179-87.

Murata Y., Rosell A., Scannevin R. H., Rhodes K. J., Wang X. Y. and Lo E. H. (2008) Extension of the thrombolytic time window with minocycline in experimental stroke. Stroke 39, 3372-33774 
Murray, A. J., Woloszynowska-Fraser, M. U., Ansel-Bollepalli, L., Cole, K. L., Foggetti, A., Crouch, B., et al. (2015). Parvalbumin-positive interneurons of the prefrontal cortex support working memory and cognitive flexibility. Sci. Rep. 5:16778. doi: 10.1038/srep16778

Nagy V., Bozdagi O. and Huntley G. W. (2007) The extracellular protease matrix metalloproteinase9 is activated by inhibitory avoidance learning and required for long-term memory. Learn. Mem. 14, 655-664

Nagy V., Bozdagi O., Matynia A. et al. (2006) Matrix metalloproteinase-9 is required for hippocampal late-phase longterm potentiation and memory. J. Neurosci. 26, 1923-1934.

Nagy, V., Bozdagi, O., Matynia, A., Balcerzyk, M., Okulski, P., Dzwonek, J., et al. (2006). Matrix metalloproteinase-9 is required for hippocampal late-phase long-term potentiation and memory. J. Neurosci. 26, 1923-1934. doi: 10.1523/JNEUROSCI.4359-05.2006

Nebel R, Aggarwal N, Barnes L, Gallagher A Goldstein J Kantarci K et al., (2018) Understanding the impact of sex and gender in Alzheimer's disease: A call to action. Alzheimers Dementia. $14: 1171-1183$

Nguyen TV, et al. (2005) Androgens activate mitogen-activated protein kinase signaling: role in neuroprotection. J Neurochem. 94:1639-51. [PubMed: 16011741]

NIH Revitalization Act. Available at: https://orwh.od.nih.gov/sites/orwh/files/docs/NIHRevitalization-Act-1993.pdf

Nilsen J, Brinton RD. (2002) Impact of progestins on estradiol potentiation of the glutamate calcium response. Neuroreport. 13:825-30. [PubMed: 11997695] 
Nilsen J, Brinton RD. (2002) Impact of progestins on estrogen-induced neuroprotection: synergy by progesterone and 19-norprogesterone and antagonism by medroxyprogesterone acetate. Endocrinology. 143:205-12. [PubMed: 11751611]

Nilsen J, Brinton RD. (2003) Divergent impact of progesterone and medroxyprogesterone acetate (Provera) on nuclear mitogen-activated protein kinase signaling. Proc Natl Acad Sci U S A. 100:10506-11. [PubMed: 12925744]

Ning M., Furie K. L., Koroshetz W. J., Lee H., Barron M., Lederer M., et al. (2006). Association between tPA therapy and raised early matrix metalloproteinase-9 in acute stroke. Neurology 66, 1550-1555. doi:10.1212/01.wnl.0000216133.98416.b4

Nord C, Sundqvist J, Andersson E, Fried G. (2010) Analysis of estrogen regulation of alpha-, betaand gamma-secretase gene and protein expression in cultured human neuronal and glial cells. Neurodegener. Dis., 7:349-364

Nübling, G., Levin, J., Bader, B., Israel, L., Bötzel, K., Lorenzl, S., Giese, A. (2012). Limited cleavage of tau with matrix-metalloproteinase MMP-9, but not MMP-3, enhances tau oligomer formation. Experimental Neurology. 237:470-476.

Oddo S., Caccamo A., Shepherd J. D., Murphy M. P., Golde T. E., Kayed R., Metherate R., Mattson M. P., Akbari Y. and LaFerla F. M. (2003) Triple-transgenic model of Alzheimer's disease with plaques and tangles: intracellular Abeta and synaptic dysfunction. Neuron 39, 409-421.

Ogata, Y., Itoh, Y., and Nagase, H. (1995). Steps involved in activation of the pro-matrix metalloproteinase 9 (progelatinase B)-tissue inhibitor of metalloproteinases-1 complex by 
4-aminophenylmercuric acetate and proteinases. J. Biol. Chem. 270, 18506-18511. doi:

10.1074/jbc.270.31.18506

Oh LY, Larsen PH, Krekoski CA, Edwards DR, Donovan F, Werb Z, Yong VW. (1999). Matrix metalloproteinase-9/gelatinase B is required for process outgrowth by oligodendrocytes. J Neurosci 19: 8464-8475.

Okulski P., Jay T. M., Jaworski J. et al. (2007) TIMP-1 abolishes MMP9-dependent long-lasting longterm potentiation in the prefrontal cortex. Biol. Psychiatry 62, 359-363

Olanrewaju, O.A., Alabi, A. M., and Akhabue Kenneth, O. (2013). Evaluation of memory status following administration of depo-provera in female wistar rats. Niger. J. Exp. Clin. Biosci. 1, 14-17. doi: 10.4103/2348-0149.123957

Oliveira SM, Ribeiro CA, Cardoso I, Saraiva MJ. (2011) Gender-dependent transthyretin modulation of brain amyloid-beta levels: evidence from a mouse model of Alzheimer's disease. J. Alzheimers Dis., 27:429-439

Oliveira-Silva, P., Jurgilas, P. B., Trindade, P., Campello-Costa, P., Perales, J., Savino, W., et al. (2007). Matrix metalloproteinase-9 is involved in the development and plasticity of retinotectal projections in rats. Neuroimmunomodulation 14, 144-149. doi: $10.1159 / 000110638$

P. Micevych, K. Sinchak. (2008) Estradiol regulation of progesterone synthesis in the brain. Mol. Cell Endocrinol., 290:44-50

P. Porcu, M.C. Mostallino, C. Sogliano, F. Santoru, R. Berretti, A. Concas (2012) Long-term administration with levonorgestrel decreases allopregnanolone levels and alters GABAA 
receptor subunit expression and anxiety-like behavior. Pharmacol. Biochem. Behav., 102, pp. 366-372.

Paganini-Hill A, Henderson VW. (1996) Estrogen replacement therapy and risk of Alzheimer disease. Arch Intern Med. 156:2213-2217.

Paliogianni, F., Raptis, A., Ahuja, S. S., Najjar, S. M., and Boumpas, D. T. (1993). Negative transcriptional regulation of human interleukin 2 (IL-2) gene by glucocorticoids through interference with nuclear transcription factors AP-1 and NF-AT. J. Clin. Invest. 91, 14811489. doi: $10.1172 /$ jci116353

Park SY, Tournell C, Sinjoanu RC, Ferreira A. (2007), Caspase-3- and calpain-mediated tau cleavage are differentially prevented by estrogen and testosterone in beta-amyloid-treated hippocampal neurons. Neuroscience. 144:119-27. [PubMed: 17055174]

Park, M. T., Cha, H. J., Jeong, J. W., Kim, S. I., Chung, H. Y., Kim, N. D., et al. (1999). Glucocorticoid receptor-induced down-regulation of MMP-9 by ginseng components, PD and PT contributes to inhibition of the invasive capacity of HT1080 human fibrosarcoma cells. Mol. Cells 9, 476-483.

Pasqualini, JR. (1996) Progestins: present and future. Journal of Steroid Biochemistry and Molecular Biology5-6357-363. doi:10.1016/S0960-0760(96)00183-5

Patel N, Hoang D, Miller N, Ansaloni S, Huang Q, Rogers JT, et al. (2008) MicroRNAs can regulate human APP levels. Mol Neurodegener 3:10.

Pavlaki M, Cao J, Hymowitz M, Chen WT, Bahou W, Zucker S. (2002) A conserved sequence within the propeptide domain of membrane type 1 matrix metalloproteinase is critical for function as an intramolecular chaperone. J Biol Chem 277: 2740-2749. 
Paylor JW, Wendlandt E, Freeman TS, Greba Q, Marks WN, Howland JG, Winship IR. (2018)

Impaired cognitive function after perineuronal net degradation in the medial prefrontal

cortex. Eneuro 5:ENEURO.0253-18.2018. doi:10.1523/ENEURO.0253-18.2018

pmid:30627657

Perl DP. (2010) Neuropathology of Alzheimer's disease. Mt Sinai J Med. 77:32-42. doi:

10.1002/msj.20157.

Perry RJ, Hodges JR. (1999) Attention and executive deficits in Alzheimer's disease. A critical review.

Brain, 122:383-404

Petrea RE, Beiser AS, Seshadri S, et al. (2009) Gender differences in stroke incidence and poststroke disability in the Framingham Heart Study. Stroke. 40:1032-1037.

Pettus EH, Wright DW, Stein DG, Hoffman SW. (2005) Progesterone treatment inhibits the inflammatory agents that accompany traumatic brain injury. Brain Res. 1049:112-9.

[PubMed: 15932748]

Pike C. J. (2017) Sex and the development of Alzheimer's disease. J. Neurosci. Res. 95, 671-680.

Pike CJ, Carroll JC, Rosario ER, Barron A. (2009) Protective actions of sex steroid hormones in Alzheimer's disease. Front Neuroendocrinol. 30:239-258.

Pike CJ. (2001) Testosterone attenuates beta-amyloid toxicity in cultured hippocampal neurons. Brain Res. 919:160-5. [PubMed: 11689174] 
Pluta R., Furmaga-Jablonska W., Maciejewski R., Ulamek-Koziol M., Jablonski M. (2013) Brain ischemia activates beta- and gamma-secretase cleavage of amyloid precursor protein: significance in sporadic Alzheimer's disease. Mol. Neurobiol. 47:425-434.

Postina R. (2008) A closer look at alpha-secretase. Curr Alzheimer Res 5: 179-86.

Prangkio, P., Yusko, E. C., Sept, D., Yang, J., and Mayer, M. (2012). Multivariate analyses of amyloid$\beta$ oligomer populations indicate a connection between pore formation and cytotoxicity. PLoS One 7:e47261. doi: 10.1371/journal.pone.0047261

Proc. Natl. Acad. Sci. U.S.A, 97 (2000), pp. 1202-1205

Pryor, N. E., Moss, M. A., and Hestekin, C. N. (2012). Unraveling the early events of amyloid- $\beta$ protein $(A \beta)$ aggregation: techniques for the determination of $A \beta$ aggregate size. Int. J. Mol. Sci. 13, 3038-3072. doi: 10.3390/ijms13033038

Quintela T, Gonçalves I, Baltazar G, Alves CH, Saraiva MJ, Santos CRA. (2009) 17beta-estradiol induces transthyretin expression in murine choroid plexus via an estrogen receptor dependent pathway. Cell Mol Neurobiol. 29(4):475-83. doi: 10.1007/s10571-008-9339-1.

R. D. Bell, E. A. Winkler, I. Singh et al., (2012) Apolipoprotein e controls cerebrovascular integrity via cyclophilin A," Nature. 485:512-516.

Ragozzino, M. E., Detrick, S., and Kesner, R. P. (1999). Involvement of the prelimbic-infralimbic areas of the rodent prefrontal cortex in behavioral flexibility for place and response learning. J. Neurosci. 19, 4585-4594. doi: 10.1523/jneurosci.19-11-04585.1999

Rahman A, Jackson H, Hristov H, Isaacson R, Saif N, Shetty T, Etingin O, Henchcliffe C, Brinton R, Mosconi L. (2019) Sex and Gender Driven Modifiers of Alzheimer's: The Role for Estrogenic 
Control Across Age, Race, Medical, and Lifestyle Risks. Front. Aging Neurosci. 11:315. doi:10.3389/fnagi.2019.00315

Rapkin A.J., M. Morgan, C. Sogliano, G. Biggio, A. Concas (2006) Decreased neuroactive steroids induced by combined oral contraceptive pills are not associated with mood changes. Fertil. Steril., 5, pp. 1371-1378.

Rapp S, Espeland M, Shumaker S, Henderson V, Brunner R, et al., (2003) Effect of Estrogen Plus Progestin on Global Cognitive Function in Postmenopausal Women. JAMA. 289:2663-2672.

Reddy, D.S. (2010). Neurosteroids: endogenous role in the human brain and therapeutic potentials. Prog. Brain Red., 186:112-137.

Reijerkerk A., Kooij G., van der Pol S. M., Khazen S., Dijkstra C. D. and de Vries H. E. (2006) Diapedesis of monocytes is associated with MMP-mediated occludin disappearance in brain endothelial cells. FASEB J. 20, 2550-2552

Reinhard, S. M., Razak, K., and Ethell, I. M. (2015). A delicate balance: role of MMP-9 in brain development and pathophysiology of neurodevelopmental disorders. Front. Cell. Neurosci. 9:280. doi: 10.3389/fncel.2015.00280

Reitz, C., vanRooij, FJA, Soaresetal, HD. (2010) Matrix metal- loproteinase 3 haplotypes and plasma amyloid beta levels: the Rotterdam study. Neurobiology of Aging. 1:715- 718.

Richardson, D. W., and Dodge, G. R. (2003). Dose dependant effects of corticosteroids on the expression of matrix-related genes in normal and cytokine-treated articular chondrocytes. Inflamm. Res. 52, 39-49. doi: 10.1007/s000110300012 
Ridnour L. A., Dhanapal S., Hoos M. et al. (2012) Nitric oxide-mediated regulation of beta-amyloid clearance via alterations of MMP-9/ TIMP-1. J. Neurochem. 123, 736-749

Rivera S., Khrestchatisky M., Kaczmarek L., Rosenberg G. A. and Jaworski D. M. (2010) Metzincin proteases and their inhibitors: foes or friends in nervous system physiology J. Neurosci. 30, 15337-15357.

Robe, P. A., Bentires-Alj, M., Bonif, M., Rogister, B., Deprez, M., Haddada, H., et al. (2004). In vitro and in vivo activity of the nuclear factor-kB inhibitor sulfasalazine in human glioblastomas. Clin. Cancer Res. 15, 5595-5603. doi: 10.1158/1078-0432.ccr-03-0392

Ronacher, K., Hadley, K., Avenant, C., Stubsrud, E., Simons, S. S. Jr., Louw, A., et al. (2009). Ligandselective transactivation and transrepression via the glucocorticoid receptor: role of cofactor interaction. Mol. Cell. Endocrinol. 299, 219-231. doi: 10.1016/j.mce.2008.10.008

Roof RL, Duvdevani R, Heyburn JW, Stein DG. (1996) Progesterone rapidly decreases brain edema: treatment delayed up to 24 hours is still effective. Exp Neurol.

Roof RL, Hall ED. (2000) Gender differences in acute CNS trauma and stroke: neuroprotective effects of estrogen and progesterone. J Neurotrauma. 17:367-88. [PubMed: 10833057]

Roomi, M. W., Monterrey, J. C., Kalinovsky, T., Rath, M., and Niedzwiecki, A. (2009). Distinct patterns of matrix metalloproteinase- 2 and -9 expression in normal human cell lines. Oncol. Rep. 21, 821-826. doi: 10.3892/or_00000290

Rosano GM, Webb CM, Chierchia S, Morgani GL, Gabraele M, Sarrel PM, de Ziegler D, Collins P. (2000) Natural progesterone, but not medroxyprogesterone acetate, enhances the 
beneficial effect of estrogen on exercise-induced myocardial ischemia in postmenopausal women. J Am Coll Cardiol. 36:2154-9. [PubMed: 11127455]

Rosario ER, Chang L, Head EH, Stanczyk FZ, Pike CJ. (2011) Brain levels of sex steroid hormones in men and women during normal aging and in Alzheimer's disease. Neurobiol Aging. 32:60413.

Rosario ER, et al. (2009) Age-related changes in serum and brain levels of androgens in male Brown Norway rats. Neuroreport. 20:1534-7. [PubMed: 19829160]

Rosario ER, et al. (2010) Testosterone regulation of Alzheimer-like neuropathology in male 3xTg-AD mice involves both estrogen and androgen pathways. Brain Res. 1359:281-90. [PubMed: 20807511]

Rosell A., Alvarez-Sabín J., Arenillas J. F., Rovira A., Delgado P., Fernandez-Cadenas I., et al. (2005). A matrix metalloproteinase protein array reveals a strong relation between MMP-9 and MMP-13 with diffusion-weighted image lesion increase in human stroke. Stroke 36, 14151420. 10.1161/01.STR.0000170641.01047.cc

Rosell A., Cuadrado E., Ortega-Aznar A., Hernández-Guillamon M., Lo E. H., Montaner J. (2008). MMP-9-positive neutrophil infiltration is associated to blood-brain barrier breakdown and basal lamina type IV collagen degradation during hemorrhagic transformation after human ischemic stroke. Stroke 39, 1121-1126. doi:10.1161/STROKEAHA.107.500868

Rosenberg GA, Cunningham LA, Wallace J, Alexander S, Estrada EY, Grossetete M, Razhagi A, Miller K, Gearing A. (2001). Immunohistochemistry of matrix metalloproteinases in reperfusion 
injury to rat brain: activation of MMP-9 linked to stromelysin-1 and microglia in cell cultures. Brain Res 893: 104-112.

Rosenberg, G. A., Dencoff, J. E., Correa, N. Jr., Reiners, M., and Ford, C. C. (1996). Effect of steroids on CSF matrix metalloproteinases in multiple sclerosis: relation to blood-brain barrier injury. Neurology 46, 1626-1632. doi: 10.1212/wnl.46.6.1626

Rosenblum, G., Meroueh, S., Toth, M., Fisher, J. F., Fridman, R., Mobashery, S., et al. (2007). Molecular structures and dynamics of the stepwise activation mechanism of a matrix metalloproteinase zymogen: challenging the cysteine switch dogma. J. Am. Chem. Soc. 129, 13566-13574. doi: 10.1021/ja073941l

Rosenthal SL, Bamne MN, Wang X, Berman S, Snitz BE, Klunk WE, et al. (2015) More evidence for association of a rare TREM2 mutation $(\mathrm{R} 47 \mathrm{H})$ with Alzheimer's disease risk. Neurobiol Aging 36: 2443.e21-6.

Ruitenberg A, Ott A, van Swieten JC, Hofman A, Breteler MMB. (2001) Incidence of dementia: does gender make a difference? Neurobiology of Aging. 22:575-580.

S. Guo, S. Wang, W. J. Kim et al., (2006) Effects of apoE isoforms on beta-amyloid-induced matrix metalloproteinase-9 in rat astrocytes. Brain Research. 1111:222-226

S. Horstmann, L. Budig, H. Gardner et al. (2010) Matrix metalloproteinases in peripheral blood and cerebrospinal fluid in patients with Alzheimer's disease. International Psychogeriatrics. 22:966-972.

S.S. Petanceska, V. Nagy, D. Frail, S. Gandy. (2000) Ovariectomy and $17 \beta$-estradiol modulate the levels of Alzheimer's amyloid $\beta$ peptides in brain. Exp. Gerontol., 35:1317-1325 
Sacta, M. A., Tharmalingam, B., Coppo, M., Rollins, D. A., Deochand, D. K., Benjamin, B., et al. (2018). Gene-specific mechanisms direct glucocorticoid-receptor-driven repression of inflammatory response genes in macrophages. Elife 7:e34864. doi: 10.7554/eLife.34864

Saido, T., and Leissring, M. A. (2012). Proteolytic degradation of amyloid $\beta$-protein. Cold Spring Harb. Perspect. Med. 2:a006379. doi: 10.1101/cshperspect.a006379

Salazar, M., Lerma-Ortiz, A., Hooks, G. M., Ashley, A. K., and Ashley, R. L. (2016). Progestinmediated activation of MAPK and AKT in nuclear progesterone receptor negative breast epithelial cells: the role of membrane progesterone receptors. Gene. 591, 6-13. doi: 10.1016/j.gene.2016.06.044

Salehi A., Zhang J.H., Obenaus A. (2017) Response of the cerebral vasculature following traumatic brain injury. J. Cereb. Blood Flow Metab.37:2320-2339.

Sampedro F, Vilaplana E, de Leon MJ et al. (2015) APOE-by-sex interactions on brain structure and metabolism in healthy elderly controls. Oncotarget 6, 26663-26674.

Sandoval K. E., Witt K. A. (2008). Blood-brain barrier tight junction permeability and ischemic stroke. Neurobiol. Dis. 32, 200-219. 10.1016/j.nbd.2008.08.005

Santoru F, Berretti R, Locci A, Porcu P, Concas A (2014) Decreased allopregnanolone induced by hormonal contraceptives is associated with a reduction in social behavior and sexual motivation in female rats. Psyhcopharmacology (Berline) 231, 3351-3364.

Sato T, Koike L, Miyata Y, Hirata M, Mimaki Y, Sashida Y, Yano M, Ito A. (2002). Inhibition of activator protein-1 binding activity and phosphatidylinositol 3-kinase pathway by nobiletin, a polymethoxy flavonoid, results in augmentation of tissue inhibitor of metalloproteinases-1 
production and suppression of production of matrix metalloproteinases-1 and -9 in human fibrosarcoma HT-1080 cells. Cancer Res 62: 1025- 1029.

Saunders AM, Strittmatter WJ, Schmechel D, George-Hyslop PH, Pericak-Vance MA, Joo SH, et al. (1993). Association of apolipoprotein E allele epsilon 4 with late-onset familial and sporadic Alzheimer's disease. Neurology 43: 1467-1472.

Scheltens P, Blennow K, Breteler MM, de Strooper B, Frisoni GB, Salloway S, Van der Flier WM (2016). Alzheimer's disease. Lancet 388, 505-517

Schindler AE, Campagnoli C, Druckmann R, Huber J, Pasqualini JR, Schweppe KW, Thijssen JH (2003) Classification and pharmacology of progestins. Maturitas 46(Suppl 1):S7-S16

Schmidt R, Kienbacher E, Benke T, Dal-Bianco P, Delazer M, Ladurner G, Jellinger K, Marksteiner J, Ransmayr G, Schmidt H, Stogmann E, Friedrich J, Wehringer C. (2008) Sex differences in Alzheimer's disease. Neuropsychiatr. 22:1-15.

Schonrock N, Matamales M, Ittner LM, Gotz J. (2012) MicroRNA networks surrounding APP and amyloid-beta metabolism-- implications for Alzheimer's disease. Exp Neurol 235: 447-54.

Schutz A, Schneidenbach D, Aust G, Tannapfel A, Steinert M, Wittekind C. (2002) Differential Expression and Activity Status of MMP-1, MMP-2 and MMP-9 in Tumor and Stromal Cells of Squamous Cell Carcinomas of the Lung. Tumour Biol 23: 179-184.

Sekine-Aizawa Y, Hama E, Watanabe K, Tsubuki S, Kanai-Azuma M, Kanai Y, Arai H, Aizawa H, Iwata N, Saido TC. (2001) Matrix metalloproteinase (MMP) system in brain: identification and characterization of brain-specific MMP highly expressed in cerebellum. Eur J Neurosci 13: 935-948. 
Seshadri S, Fitzpatrick AL, Ikram MA, DeStefano AL, Gudnason V, Boada M, et al. (2010) Genomewide analysis of genetic loci associated with Alzheimer disease. JAMA 303: 1832-40.

Shen R, Sumitomo M, Dai J, Hardy DO, Navarro D, Usmani B, Papandreou CN, Hersh LB, Shipp MA, Freedman LP, Nanus DM. (2000) Identification and characterization of two androgen response regions in the human neutral endopeptidase gene. Mol Cell Endocrinol. 170:13142. [PubMed: 11162897]

Sherrington R, Rogaev EI, Liang Y, Rogaeva EA, Levesque G, Ikeda M, et al. (1993) Cloning of a gene bearing missense mutations in early onset familial Alzheimer's disease. Nature 375: 754-60.

Sherwin BB. (2003). Estrogen and cognitive functioning in women. Endocr Rev;24(2):133-51

Sherwin BB. (2012). Estrogen and cognitive functioning in women: lessons we have learned. Behav Neurosci;126(1):123-7.

Shrimanker, K., B. N. Saxena, and K. A. Fotherby. (1978) A radioimmunoassay for serum medroxyprogesterone acetate.J. Steroid Biochem. 9:359-363.

Shumaker S, Legault C, Kuller L, Rapp SR, Thal L, Lane DS, et al. (2004) Conjugated equine estrogens and incidence of probable dementia and mild cognitive impairment in postmenopausal women: Women's Health Initiative Memory Study. JAMA. 291:2947-58.

Shumaker, S. A., Legault, C., Kuller, L., Rapp, S. R., Thal, L., Lane, D. S., et al. (2004). Conjugated equine estrogens and incidence of probable dementia and mild cognitive impairment in postmenopausal women: women's health initiative memory study. JAMA 291, 2947-2958. doi: 10.1001/jama.291.24.2947 
Shumaker, S. A., Legault, C., Rapp, S. R., Thal, L., Wallace, R. B., Ockene, J. K., et al. (2003). Estrogen plus progestin and the incidence of dementia and mild cognitive impairment in postmenopausal women. JAMA 289, 2651-2652. doi: 10.1001/jama.289.20.2651

Silva D.F., Selfridge J.E., Lu J., E. L., Roy N., Hutfles L., Burns J.M., Michaelis E.K., Yan S., Cardoso S.M., Swerdlow R.H. (2013) Bioenergetic flux, mitochondrial mass and mitochondrial morphology dynamics in AD and MCl cybrid cell lines. Hum. Mol. Genet. 22:3931-3946.

Simoncini, T., Mannella, P., Fornari, L., Caruso, A., Willis, M. Y., Garibaldi, S., et al. (2004). Differential signal transduction of progesterone and medroxyprogesterone acetate in human endothelial cells. Endocrinology 145, 5745-5756. doi: 10.1210/en.2004-0510

Simpkins JW, Singh M, Brock C, Etgen AM. (2012) Neuroprotection and estrogen receptors. Neuroendocrinology. 96:119-130.

Simpkins, JW, Perez, E, Wang, X, Yang, S, Wen, Y, Singh, M. (2009) The potential for estrogens in preventing Alzheimer's disease. Ther. Adv. Neurol. Disord., 2:31-49

Simpkins, JW, Yi, KD, Yang, SH, Dykens, JA. (2010) Mitochondrial mechanisms of estrogen neuroprotection. Biochim. Biophys. Acta, 1800:1113-1120

Sinforiani E, Citterio A, Zucchella C, et al. (2010) Impact of gender differences on the outcome of Alzheimer's disease. Dementia. 30:147-54.

Singh, M., Su, C., and Ng, S. (2013). Non-genomic mechanisms of progesterone action in the brain. Front. Neurosci. 7:159. doi: 10.3389/fnins.2013.00159.

Singh-Manoux A, Dugravot A, Fournier A et al. (2017) Trajectories of Depressive Symptoms Before Diagnosis of Dementia: A 28-Year Follow-up Study. JAMA Psychiatry 74, 712-718. 
Sitruk-Ware R (2000) Therapeutic use of progestins: practical recommendations. In Sitruk-Ware R and Mishell DR Jr (eds), Progestins and Antiprogestins in Clinical Pratice. Marcel Dekker, Inc., New York, 341-353

Slaker M Churchill L Todd R Blacktop J Zuloaga D Raber J Darling R Brown T Sorg B. (2015) Removal of perineuronal nets in the medial prefrontal cortex impairs the acquisition and reconsolidation of a cocaine-induced conditioned place preference memory. Journal of Neuroscience. 35:4190-4202

Snowdon, D.A, Greiner, L.H., Mortimer, J.A., Riley, K.P., Greiner, P.A., Markesbery, W.R. (1997) Brain infarction and the clinical expression of Alzheimer disease. The Nun Study. JAMA, 277:813-817

Sohrabji, F. (2007). Guarding the Blood-Brain Barrier: A Role of Estrogen in the Etiology of Neurodegenerative Disease. Gene Expr. 13:311-319.

Song, E. S., Rodgers, D. W., and Hersh, L. B. (2018). Insulin-degrading enzyme is not secreted from cultured cells. Sci. Rep. 8:2335. doi: 10.1038/s41598-018-20597-6

Sorg B. A., Berretta S., Blacktop J. M., et al. (2016) Casting a wide net: role of perineuronal nets in neural plasticity. The Journal of Neuroscience. 36(45):11459-11468. doi:10.1523/JNEUROSCI.2351-16.2016.

Sotgiu S., Zanda B., Marchetti B., Fois M. L., Arru G., Pes G. M., et al. (2006). Inflammatory biomarkers in blood of patients with acute brain ischemia. Eur. J. Neurol. 13, 505-513. 10.1111/j.1468-1331.2006.01280.x

Stawarski M., Stefaniuk M. and Wlodarczyk J. (2014) Matrix metalloproteinase-9 involvement in the structural plasticity of dendritic spines. Front. Neuroanat. 8, 68. 
Stern Y. (2009) Cognitive reserve. Neuropsychologia. 47: 2015-28

Strittmatter WJ, Weisgraber KH, Huang DY, Dong LM, Salvesen GS, Pericak-Vance M, et al. (1993) Binding of human apolipoprotein $\mathrm{E}$ to synthetic amyloid beta peptide: isoform-specific effects and implications for late-onset Alzheimer disease. Proc Natl Acad Sci USA 90: 80988102

Sturchler-Pierrat C, Staufenbiel M (2000). Pathogenic mechanisms of Alzheimer's disease analyzed in the APP23 transgenic mouse model. Ann N Y Acad Sci 920: 134- 139.

Su, C., Cunningham, R. L., Rybalchenko, N., and Singh, M. (2012a). Progesterone increases the release of brain-derived neurotrophic factor from glia via progesterone receptor membrane component 1 (Pgrmc1)-dependent ERK5 signaling. Endocrinology 153, 4389-4400. doi: 10.1210/en.2011-2177

Su, C., Rybalchenko, N., Schreihofer, D. A., Singh, M., Abbassi, B., and Cunningham, R. L. (2012b). Cell models for the study of sex steroid hormone neurobiology. J. Steroids Horm. Sci. S2:003. doi: 10.4172/2157-7536.s2-003

Surjit, M., Ganti, K. P., Mukherji, A., Ye, T., Hua, G., Metzger, D., et al. (2011). Widespread negative response elements mediate direct repression by agonist-liganded glucocorticoid receptor. Cell 145, 224-241. doi: 10.1016/j.cell.2011.03.027

Suzuki S, Brown CM, and Wise PM (2009) Neuroprotective effects of estrogens following ischemic stroke. Front Neuroendocrinol 30:201-211.

Svedin P., Hagberg H., Sävman K., Zhu C., Mallard C. (2007). Matrix metalloproteinase-9 gene knock-out protects the immature brain after cerebral hypoxia-ischemia. J. Neurosci. 27, 1511-1518. 10.1523/JNEUROSCI.4391-06.2007 
Swerdlow R.H. (2018) Mitochondria and mitochondrial cascades in Alzheimer's disease. J.

Alzheimers Dis.

Szarka N., Pabbidi M.R., Amrein K., Czeiter E., Berta G., Pohoczky K., Helyes Z., Ungvari Z., Koller A., Buki A., Toth P. (2018) Traumatic brain injury impairs myogenic constriction of cerebral arteries: role of mitochondria-derived $\mathrm{H} 2 \mathrm{O} 2$ and TRPV4-dependent activation of BKCa channels. J. Neurotrauma.

Talamagas AA, Efthimiopoulos S, Tsilibary EC, Figueiredo-Pereira ME, and Tzinia AK. (2007) Abeta (1-40)-induced secretionofmatrixmetalloproteinase-9 results in SAPP release by association with cell surface APP. Neurobiology of Disease, 28:04-315.

Talamagas, A. A., Efthimiopoulos, S., Tsilibary, E. C., Figueiredo-Pereira, M. E., and Tzinia, A. K. (2007). A (1-40)-induced secretion of matrix metalloproteinase-9 results in SAPP $\alpha$ release by association with cell surface APP. Neurobiol. Dis. 28, 304-315. doi: 10.1016/j.nbd.2007.07.016

Tan RS. (2013) Testosterone effect on brain metabolism in elderly patients with Alzheimer's disease: comparing two cases at different disease stages. Aging clinical and experimental research. 25:343-7. [PubMed: 23740590]

Tang MX, Jacobs D, Stern Y, Marder K, Schofield P, Gurland B, Andrews H, Mayeux R. (1996) Effect of oestrogen during menopause on risk and age at onset of Alzheimer's disease. Lancet. 348:429-432.

Tanney DC, Feng L, Pollock AS, Lovett DH. (1998). Regulated expression of matrix metalloproteinases and TIMP in nephrogenesis. Dev Dyn 213: 121-129. 
Taraborrelli, S. (2015). Physiology, production and action of progesterone. Acta Obstet Gynecol Scand. 94: 8- 16.

Tarasoff-Conway J.M., Carare R.O., Osorio R.S., Glodzik L., Butler T., Fieremans E., Axel L., Rusinek H., Nicholson C., Zlokovic B.V., Frangione B., Blennow K., Menard J., Zetterberg H., Wisniewski T., DE Leon M.J. (2015) Clearance systems in the brain-implications for Alzheimer disease. Nat. Rev. Neurol. 11:457-470.

Thakur MK, Mani ST. (2005) Estradiol regulates APP mRNA alternative splicing in the mice brain cortex. Neurosci. Lett., 381:154-157

Thomas P , Pang Y. (2012) Membrane progesterone receptors (mPRs): evidence for neuroprotective, neurosteroid signaling and neuroendocrine functions in neuronal cells. Neuroendocrinology 96:162-171.

Thomas P, Pang Y, Dong J, Groenen P, Kelder J, Vlieg J.d., Zhu Y, Tubbs C. (2007). Steroid and G protein binding characteristics of the seatrout and human progestin membrane receptor a subtypes and their evolutionary origins. Endocrinology 148, 705-718.

Tomasicchio, M., Avenant, C., Du Toit, A., Ray, R. M., and Hapgood, J. P. (2013). The progestin-only contraceptive medroxyprogesterone acetate, but not norethisterone acetate, enhances HIV-1 Vpr-mediated apoptosis in human CD4+ T cells through the glucocorticoid receptor. PLoS One 8:e62895. doi: 10.1371/journal.pone.0062895

Tosto, G., and Reitz, C. (2013). Genome-wide association studies in Alzheimer's disease: a review. Curr. Neurol. Neurosci. Rep. 13:381. doi: 10.1007/s11910-013-0381-0

Trevor, J. L., and Deshane, J. S. (2014). Refractory asthma: mechanisms, targets, and therapy. Allergy 69, 817-827. doi: 10.1111/all.12412 
Tschanz JT, Corcoran CD, Schwartz S, Treiber K, Green RC, Norton MC, et al. (2011) Progression of cognitive, functional, and neuropsychiatric symptom domains in a population cohort with Alzheimer dementia: the Cache County Dementia Progression study. The American Journal of Geriatric Psychiatry. 19:532-542.

Tsubuki S, Takaki Y, Saido TC. (2003) Dutch, Flemish, Italian, and Arctic mutations of APP and resistance of Abeta to physiologically relevant proteolytic degradation. Lancet 361: 1957-8.

Uchoa, M. F., Moser, V. A., and Pike, C. J. (2016). Interactions between inflammation, sex steroids, and Alzheimer's disease risk factors. Front. Neuroendocrinol. 43, 60-82. doi: 10.1016/j.yfrne.2016.09.001

United Nations, Department of Economic and Social Affairs, Population Division (2013). World Contraceptive Patterns

Vafadari B., Salamian A., and Kaczmarek L. (2016). MMP-9 in translation: from molecule to brain physiology, pathology, and therapy. J. Neurochem. 139, 91-114. doi: 10.1111/jnc.13415

Vaillant C, Didier-Bazes M, Hutter A, Belin MF, Thomasset N. (1999). Spatiotemporal expression patterns of metalloproteinases and their inhibitors in the postnatal developing rat cerebellum. J Neurosci 19: 4994-5004.

Vallee BL, Auld DS (1990) Zinc coordination, function, and structure of zinc enzymes and other proteins. Biochemistry 29: 5647-5659.

Van den Steen, P. E., Dubois, B., Nelissen, I., Rudd, P. M., Dwek, R. A., and Opdenakker, G. (2002). Biochemistry and molecular biology of gelatinase B or matrix metalloproteinase-9 (MMP9). Crit. Rev. Biochem. Mol. Biol. 37, 375-536. doi: 10.1080/10409230290771546 
Van den Steen, P., Dubois, B., Nelissen, I., Dwek, R., and Opdenakker, G. (2002). Biochemistry and molecular biology of gelatinase B or matrix metalloproteinase-9 (MMP-9). Crit. Rev. Biochem. Mol. Biol. 37, 375-536. doi: 10.1080/10409230290771546

Vandooren, J., Knoops, S., Aldinucci Buzzo, J. L., Boon, L., Martens, E., Opdenakker, G., et al. (2017). Differential inhibition of activity, activation and gene expression of MMP-9 in THP-1 cells by azithromycin and minocycline versus bortezomib: a comparative study. PLoS One 12:e0174853. doi: 10.1371/journal.pone.0174853

Verdile G, Laws SM, Henley D, Ames D, Bush Al, Ellis KA, Faux NG, Gupta VB, Li QX, Masters CL, Pike KE, Rowe CC, Szoeke C, Taddei K, Villemagne VL, Martins RN. (2012). ARG. for the, Associations between gonadotropins, testosterone and beta amyloid in men at risk of Alzheimer's disease. Mol Psychiatry.

Verslegers M., Lemmens K., Van Hove I. and Moons L. (2013) Matrix metalloproteinase-2 and -9 as promising benefactors in development, plasticity and repair of the nervous system. Prog. Neurobiol. 105, 60-78

Vest, R. S., and Pike, C. J. (2013). Gender, sex steroid hormones, and Alzheimer's disease. Horm. Behav. 63, 301-307. doi: 10.1016/j.yhbeh.2012.04.006

Vilardo E, Barbato C, Ciotti M, Cogoni C, Ruberti F. (2010) MicroRNA101 regulates amyloid precursor protein expression in hippocampal neurons. J Biol Chem 285: 18344-51.

Vukasovic I., Tesija-Kuna A., Topic E., Supanc V., Demarin V., Petrovcic M. (2006). Matrix metalloproteinases and their inhibitors in different acute stroke subtypes. Clin. Chem. Lab. Med. 44, 428-434. 10.1515/cclm.2006.079 
Wahjoepramono EJ, Wijaya LK, Taddei K, Martins G, Howard M, de Ruyck K, Bates K, et al. (2008).

Distinct effects of testosterone on plasma and cerebrospinal fluid amyloid beta levels. J Alzheimers Dis. 15:129-37. [PubMed: 18780973]

Wang C. X., Ding X., Noor R., Pegg C., He C., Shuaib A. (2009). Rosiglitazone alone or in combination with tissue plasminogen activator improves ischemic brain injury in an embolic model in rats. J. Cereb. Blood Flow Metab. 29, 1683-1694. 10.1038/jcbfm.2009.87

Wang JM, Johnston PB, Ball BG, Brinton RD. (2005). The neurosteroid allopregnanolone promotes proliferation of rodent and human neural progenitor cells and regulates cell-cycle gene and protein expression. J Neurosci.25:4706-4718.

Wang JM, Singh C, Liu L, Irwin RW, Chen S, Chung EJ, Thompson RF, Brinton RD. (2010). Allopregnanolone reverses neurogenic and cognitive deficits in mouse model of Alzheimer's disease. Proc Natl Acad Sci U S A.107(14):6498-503.

Wang X, Mori T, Jung JC, Fini ME, Lo EH. (2002). Secretion of matrix metalloproteinase-2 and -9 after mechanical trauma injury in rat cortical cultures and involvement of MAP kinase. J Neurotrauma 19: 615-625.

Wang, X.-X., Tan, M.-S., Yu, J.-T., and Tan, L. (2014). Matrix metalloproteinases and their multiple roles in Alzheimer's disease. Biomed. Red. Int. 2014:908636. doi: 10.1155/2014/908636

Wassertheil-Smoller S, Hendrix S, Limacher M, et al. (2003). Effect of estrogen plus progestin on stroke in post-menopausal women: the Women's Health Initiative: a randomized trial JAMA. 289:2673-2684 
Watson, L. C., Kuchenbecker, K. M., Schiller, B. J., Gross, J. D., Pufall, M. A., and Yamamoto, K. R. (2013). The glucocorticoid receptor dimer interface allosterically transmits. Nat. Struct. Mol. Biol. 20, 876-883. doi: 10.1038/nsmb.2595

Webster, J. C., and Cidlowski, J. A. (1999). Mechanisms of glucocorticoid-receptor-mediated repression of gene expression. Trends Endocrinol. Metab. 10, 396-402. doi: 10.1016/s10432760(99)00186-1

Wegiel, J., Wang, K. C., Tarnawski, M., and Lach, B. (2000). Microglial cells are the driving force in fibrillar plaque formation, whereas astrocytes are a leading factor in plaque degradation. Acta Neuropathol. 100, 356-364. doi: 10.1007/s004010000199

Wei, G., and Shea, J. E. (2006). Effects of solvent on the structure of the Alzheimer amyloid-beta (25-35) peptide. Biophys. J. 91, 1638-1647. doi: 10.1529/biophysj.105.079186

Weikum, E. R., de Vera, I. M. S., Nwachukwu, J. C., Hudson, W. H., Nettles, K. W., Kojetin, D. J., et al. (2017). Tethering not required: the glucocorticoid receptor binds directly to activator protein-1 recognition motifs to repress inflammatory genes. Nucleic Acids Res. 45, 85968608. doi: 10.1093/nar/gkx509

Wen J., Qian S., Yang Q., Deng L., Mo, Y. \& Yu, Y. (2014). Overexpression of netrin-1 increases the expression of tight junction-associated proteins, claudin-5, occludin, and ZO-1, following traumatic brain injury in rats. Exp. Ther. Med. 8:881-886.

Wen T, Binder D, Ethell I, Razak K. (2018) The Perineuronal 'Safety' Net? Perineuronal Net Abnormalities in Neurological Disorders. Frontiers in Molecular Neuroscience. 11:270

White LA, Mitchell TI, Brinckerhoff CE. (2000). Transforming growth factor beta inhibitory element in the rabbit matrix metalloproteinase-1 (collagenase-1) gene functions as a repressor of constitutive transcription. Biochim Biophys Acta 1490: 259- 268. 
Whitmer RA, Quesenberry Jr CP, Zhou J, Yaffe K. (2011) Timing of hormone therapy and dementia: the critical window theory revisited Ann. Neurol., 69:163-169

Wiera G., Wojtowicz T., Lebida K. et al. (2012) Long term potentiation affects intracellular metalloproteinases activity in the mossy fiberCA3 pathway. Mol. Cell Neurosci. 50, 147-159.

Wilkaniec A, Czapski GA, Adamczyk A. (2016) CDK5 at crossroads of protein oligomerization in neurodegenerative diseases: facts and hypotheses. J Neurochem 136: 222-33.

Wilson AC., Clemente L., Liu T., Bowen RL., Meethal SV., Atwood CS. (2008). Reproductive hormones regulate the selective permeability of the blood-brain-barrier. Biochimica et Biophysica Acta (BBA) - Molecular Basis of Disease. 1782: 401-407.

Winneker RC, Bitran D, Zhang Z. (2003) The preclinical biology of a new potent and selective progestin: trimegestone. Steroids. 68:915-920.

Wlodarczyk J., Mukhina I., Kaczmarek L., Dityatev A. (2011) Extracellular matrix molecules, their receptors, and secreted proteases in synaptic plasticity. Developmental Neurobiology. 71(11):1040-1053. doi: 10.1002/dneu.20958.

Wojtowicz T. and Mozrzymas J. W. (2010) Late phase of long-term potentiation in the mossy fiberCA3 hippocampal pathway is critically dependent on metalloproteinases activity. Hippocampus 20, 917-921.

Wolters F.J., Zonneveld H.I., Hofman A., Van Der Lugt A., Koudstaal P.J., Vernooij M.W., Ikram M.A. (2017). Heart-Brain Connection Collaborative Research, G Cerebral perfusion and the risk of dementia: a population-based study. Circulation. 136:719-728. 
Wright J. W., Meighan S. E., Murphy E. S., Holtfreter K. L., Davis C. J., Olson M. L., Benoist C. C., Muhunthan K. and Harding J. W. (2006). Habituation of the head-shake response induces changes in brain matrix metalloproteinases-3 (MMP-3) and -9. Behav. Brain Res. 174, 7885.

Xu H, Greenfield JP, Cai D, Leung LW, Kassik K, Rodriguez Boulan E, Greengard P. (2002) Hormonal control of cerebral amyloidogenesis in Alzheimer's diseases. Journal of neurochemistry. 81

Xu, T. H., Yan, Y., Kang, Y., Jiang, Y., Melcher, K., and Xu, H. E. (2016). Alzheimer's diseaseassociated mutations increase amyloid precursor protein resistance to $\gamma$-secretase cleavage and the $A \beta 42 / A \beta 40$ ratio. Cell Discov. 2:16026. doi: 10.1038/celldisc.2016.26.0004

Yan, P., Hu, X., Song, H., Yin, K., Bateman, R. J., Cirrito, J. R., et al. (2006). Matrix metalloproteinase9 degrades amyloid-fibrils in vitro and compact plaques in situ. J. Biol. Chem. 281, 2456624574. doi: $10.1074 / j b c . m 602440200$

Yang Y. and Rosenberg G. A. (2015) Matrix metalloproteinases as therapeutic targets for stroke. Brain Res. 1623, 30-38.

Yang, L. B., Lindholm, K., Yan, R., Citron, M., Xia, W., Yang, X. L., et al. (2003). Elevated $\beta$-secretase expression and enzymatic activity detected in sporadic Alzheimer disease. Nat. Med. 9, 3-4. doi: $10.1038 / \mathrm{nm} 0103-3$

Yim MH, Alkema L, Kantorova V. (2017) National, regional and global estimates and projections of the number of women aged 15 to 49 who are married or in a union, 1970-2030

Yin, K.-J., Cirrito, J. R., Yan, P., Hu, X., Xiao, Q., Pan, X., et al. (2006). Neurobiology of disease matrix metalloproteinases expressed by astrocytes mediate extracellular amyloid-peptide catabolism. J. Neurosci. 26, 10939-10948. doi: 10.1523/JNEUROSCI.2085-06.2006 
Yokoo, T., and Kitamura, M. (1996). Dual regulation of IL-1 $\beta$-mediated matrix metalloproteinase-9 expression in mesangial cells by NF-kappa B and AP-1. Am. J. Physiol. 270, F123-F130. doi: 10.1152/ajprenal.1996.270.1.f123

Yong V. W., Zabad R. K., Agrawal S., Goncalves Dasilva A. and Metz L. M. (2007) Elevation of matrix metalloproteinases (MMPs) in multiple sclerosis and impact of immunomodulators. J. Neurol. Sci. 259, 79-84

Yong, V. W. (2005). Metalloproteinases: mediators of pathology and regeneration in the CNS. Nat. Rev. Neurosci. 6, 931-944. doi: 10.1038/nrn1807

Yong, V. W. (2005). Metalloproteinases: mediators of pathology and regeneration in the CNS. Nat. Rev. Neurosci. 6, 931-944. doi: 10.1038/nrn1807

Yue X, Lu M, Lancaster T, Cao P, Honda S, Staufenbiel M, Harada N, Zhong Z, Shen Y, and Li R. (2005). Brain estrogen deficiency accelerates Abeta plaque formation in an Alzheimer's disease animal model. Proc Natl Acad Sci USA 102:19198-19203

Zandi PP, Carlson MC, Plassman BL, Welsh-Bohmer KA, Mayer LS, Steffens DC, Breitner JC. (2002) Hormone replacement therapy and incidence of Alzheimer disease in older women: the Cache County Study. JAMA. 288:2123-2129.

Zechel J, Gohil H, Lust WD, Cohen A. (2002). Alterations in matrix metalloproteinase-9 levels and tissue inhibitor of matrix metalloproteinases-1 expression in a transforming growth factorbeta transgenic model of hydrocephalus. J Neurosci Res 69: 662-668.

Zhang X. (2004) Cholinergic activity and amyloid precursor protein processing in aging and Alzheimer's disease. Curr Drug Targets CNS Neurol Disord. 3:137-52. [PubMed: 15078189] 
Zhang, X., Li, Y., Xu, H., and Zhang, Y. W. (2014). The $\mathrm{Y}$-secretase complex: from structure to function. Front. Cell. Neurosci. 8:427. doi: 10.3389/fncel.2014.00427

Zhao, L., Yao, J., Mao, Z., Chen, S., Wang, Y., and Brinton, R. D. (2011). 17 ß-Estradiol regulates insulin-degrading enzyme expression via an ERß/PI3-K pathway in hippocampus: relevance to Alzheimer's prevention. Neurobiol. Aging 32, 1949-1963. doi:

10.1016/j.neurobiolaging.2009.12.010

Zwain, I., Yen S. (1999). Neurosteroidogenesis in astrocytes, oligodendrocytes, and neurons of cerebral cortex of rat brain. Endocrinol. 140:3843-3852. 